\title{
CHARACTERIZATION AND MODELING OF AN O-BAND 1310 NM \\ SAMPLED-GRATING DISTRIBUTED BRAGG REFLECTOR (SG-DBR) \\ LASER FOR OPTICAL COHERENCE TOMOGRAPHY (OCT) \\ APPLICATIONS
}

\author{
A Thesis \\ Presented to \\ the Faculty of California Polytechnic State University \\ San Luis Obispo
}

In Partial Fulfillment

of the Requirements for the Degree

Master of Science in Electrical Engineering

by

Desmond Charles Talkington

June 2013 
(C) 2013

Desmond Charles Talkington

ALL RIGHTS RESERVED 
COMMITTEE MEMBERSHIP

TITLE: Characterization and Modeling of an OBand 1310 nm Sampled-Grating Distributed Bragg Reflector (SG-DBR) Laser for Optical Coherence Tomography (OCT) Applications

AUTHOR: Desmond Charles Talkington

DATE SUBMITTED: June 2013

COMMITTEe CHAIR: Dennis Derickson, Ph.D.

Department Chair, Electrical Engineering

COMMiTTEE MEMBER: Tina Smilkstein, Ph.D.

Assistant Professor, Electrical Engineering

COMMiTTEe MEMBER: John Sharpe, Ph.D.

Professor, Physics 


\begin{abstract}
Characterization and Modeling of an O-Band $1310 \mathrm{~nm}$ Sampled-Grating

Distributed Bragg Reflector (SG-DBR) Laser for Optical Coherence

Tomography (OCT) Applications

Desmond Charles Talkington
\end{abstract}

In this project, the performance aspects of a new early generation $1310 \mathrm{~nm}$ Sampled-Grating Distributed Bragg Reflector (SG-DBR) semiconductor laser are investigated. SG-DBR lasers are ideal for Source Swept Optical Coherence Tomography (SS-OCT), a Fourier-Domain based approach for OCT, necessitating a tunable wavelength source. Three internal sections control the frequency output for tuning, along with two amplifiers for amplitude control. These O-band SG-DBR devices are now being produced in research quantities. SG-DBR lasers have been produced at 1550 and $1600 \mathrm{~nm}$ for some times. Fundamental questions regarding the performance of the $1310 \mathrm{~nm}$ devices must be quantified. Standard metrics including the laser linewidth, amplitude modulation and frequency modulation responses are characterized. The intrinsic electrical parasitics of the laser diode segments and packaging are also investigated. In addition, testing fixture including a Thermal Electric Cooler (TEC) controller is for the characterization task. Measurements of these key metrics are essential to the enhancement of future devices, aiding in the optimization of more mature products. 


\section{Acknowledgements}

I would like to thank Dr. Dennis Derickson of the Electrical Engineering Department for his invaluable guidance and assistance throughout the project. His extensive theoretical and practical knowledge was a highly valuable resource. This project greatly expanded my interest and knowledge in the subject of not only semiconductor lasers but into the exciting field of medical imaging.

Thanks goes to my additional committee members Dr. Tina Smilkstein from Electrical Engineering, and Dr. John Sharpe from Physics. I also would like to thank Chris Chiccone, Shane O'Connor, and Brandon George for their previous work on similar SG-DBR projects at $1550 \mathrm{~nm}$. Their foundations were of great use in the development of this new research.

I appreciate the opportunity to work with Dr. Jason Ensher, Mike Crawford, and others at Insight Photonics for their support and use of the SG-DBR lasers.

Thanks to my family for their unwavering support throughout my career at Cal Poly. Without them this would not be possible.

Lastly, I would like to thank all of my friends and fellow colleagues near and far. The grad lab was a great source of camaraderie among fellow engineers. I especially enjoyed our coffee breaks for discussing our theses to whoever would listen walking around outside. 


\section{Contents}

List of Tables viii

List of Figures $\quad$ ix

1 Introduction 1

2 Application Background 3

2.1 OCT Approaches .................... 4

3 SG-DBR Tunable Laser Background $\quad 10$

3.1 Laser Physics . . . . . . . . . . . . . . . . . . . . . 10

3.2 Semiconductor Laser Diode . . . . . . . . . . . . . . . . . . . . . 13

3.3 SG-DBR Laser Structure . . . . . . . . . . . . . . . . . . . 17

3.4 Single Mirror Tuning . . . . . . . . . . . . . . . . . . . 21

3.5 Dual Mirror Tuning . . . . . . . . . . . . . . . . . 22

3.6 Phase Section Tuning . . . . . . . . . . . . . . . . . . . 23

3.7 Motivations for $\mathrm{OCT} \ldots \ldots . \ldots . \ldots 26$

4 Testing Fixture $\quad 28$

4.1 PCB Design . . . . . . . . . . . . . . . . . . . . . 28

4.2 TEC Controller . . . . . . . . . . . . . . . . 33

5 Equivalent Circuit Models $\quad 37$

5.1 Test $\operatorname{Setup} \ldots \ldots \ldots$. . . . . . . . . . . . . . 37

5.2 Front Mirror . . . . . . . . . . . . . . . . . . . . . . . . . 42

5.3 Back Mirror . . . . . . . . . . . . . . . . . . . . . 46

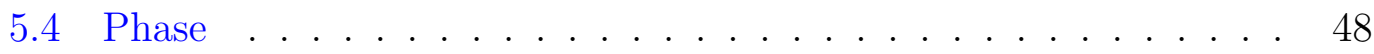


5.5 Gain . . . . . . . . . . . . . . . . 50

5.6 Semiconductor Optical Amplifier _ . . . . . . . . . . . . 52

5.7 Analysis . . . . . . . . . . . . . . . . . . . 53

6 Amplitude Modulation (AM) Bandwidth Characterization $\quad 57$

6.1 Test Setup . . . . . . . . . . . . . . . . . . . . . 58

6.2 AM Response on GAIN Section . . . . . . . . . . . . . . 61

6.3 AM Response on SOA Section . . . . . . . . . . . . . 67

7 Frequency Modulation (FM) Bandwidth Characterization $\quad 70$

7.1 Test Setup . . . . . . . . . . . . . . . . . . . . . . . 70

7.2 FM Response on Phase Section _ . . . . . . . . . . . . . 79

7.3 FM Response on Front Mirror Section _. . . . . . . . . . 88

7.4 FM Response on Back Mirror Section . . . . . . . . . . . . . . . 94

7.5 Summary of FM Responses _. . . . . . . . . . . . . . . . 99

8 Linewidth 100

8.1 Test Setup . . . . . . . . . . . . . . . . . . . . . 100

8.2 Results . . . . . . . . . . . . . . . . . . . . 103

9 Conclusion and Future Work 110

$\begin{array}{ll}\text { Bibliography } & 116\end{array}$

$\begin{array}{ll}\text { Appendices } & 120\end{array}$

A Laser Pinout. . . . . . . . . . . . . . . . . . . 120

B TEC Controller Schematic . . . . . . . . . . . . . . . 121

C PCB Gerber Files . . . . . . . . . . . . . . . . . . 123

D Bill of Materials . . . . . . . . . . . . . . . . . . . 125

E Amplitude Modulation Data . . . . . . . . . . . . . . 127 


\section{List of Tables}

2.1 Comparison Between TD-OCT, SD-OCT, and SS-OCT . . . . . 7

5.1 Front Mirror Circuit Summary . . . . . . . . . . . . . . . 44

5.2 Back Mirror Circuit Summary . . . . . . . . . . . . . . . . . . . . 46

5.3 Phase Section Circuit Summary . . . . . . . . . . . . . . . . 49

5.4 Gain Section Circuit Summary . . . . . . . . . . . . 51

5.5 SOA Section Circuit Summary . . . . . . . . . . . . 53

5.6 TDR Oscillation Frequency Comparison . . . . . . . . . . . . . 54

5.7 Inductance Induced Oscillation Comparison . . . . . . . . . . . 56

7.1 Quadrature Decision Matrix ... . . . . . . . . . 81

7.2 RC Parasitic and FM Response Limited Bandwidth Comparison . 99

9.1 Equivalent Circuit Model Summary . . . . . . . . . . . . . . 111

9.2 RC Limited Bandwidth Summary . . . . . . . . . . . . . . . . . . 112

9.3 Amplitude Modulation Response Summary . . . . . . . . . . . . . 113

9.4 Frequency Modulation Response Summary . . . . . . . . . . . . . 114

D.1 Bill of Materials for Breakout Board . . . . . . . . . . . 126 


\section{List of Figures}

2.1 Medical Imaging Technique Comparison _. . . . . . . . . . 4

2.2 Time and Fourier Domain OCT Comparison . . . . . . . . . 6

2.3 OCT Example Image of Skin . . . . . . . . . . . . . . . . . . 8

2.4 3D OCT Example Image of Skin . . . . . . . . . . . . . . 9

3.1 Generic Laser Schematic . . . . . . . . . . . . . . . . . . . . 12

3.2 Laser Emission Processes . . . . . . . . . . . . . . . . . . . . . . . 13

3.3 Semiconductor Laser Energy Diagram . . . . . . . . . . . . . . . 14

3.4 Double-Heterostructure Semiconductor Laser Profile View . . . 16

3.5 Structure of the SG-DBR Laser . . . . . . . . . . . . . . . . 19

3.6 SG-DBR Reflectivity Structure . . . . . . . . . . . . . . 20

3.7 SG-DBR Single Mirror Tuning Example . . . . . . . . . . . . . 22

3.8 SG-DBR Single Mirror Tuning Conceptual Illustration . . . . . . 23

3.9 SG-DBR Dual Mirror Tuning Conceptual Illustration . . . . . . . 24

3.10 SG-DBR Phase Tuning Example . . . . . . . . . . . . . . . 25

3.11 Measurement Motivations for SS-OCT _ . . . . . . . . . 26

4.1 Detail Structure of the SG-DBR Laser . . . . . . . . . . . 29

4.2 PCB Testing Fixture Rendering . . . . . . . . . . . . . . 32

4.3 PCB Testing Fixture Assembled . . . . . . . . . . . . . . . . 32

4.4 TEC PID Control Loop . . . . . . . . . . . . . . . . . . 35

5.1 Circuit Model TDR Test Setup . . . . . . . . . . . . . . . . 39

5.2 TDR Experimental Test Setup . . . . . . . . . . . . . . . 40 
5.3 Small Signal Diode Model . . . . . . . . . . . . . . . . . . . . 42

5.4 Front Mirror I-V . . . . . . . . . . . . . . . . . . . . 43

5.5 Front Mirror TDR Bias . . . . . . . . . . . . . . . . . 45

5.6 Back Mirror I-V . . . . . . . . . . . . . . . . . . . . 46

5.7 Back Mirror TDR Bias . . . . . . . . . . . . . . . . . 47

5.8 Phase Mirror I-V . . . . . . . . . . . . . . . . . . . . . . . . . . 48

5.9 Phase Mirror TDR Bias . . . . . . . . . . . . . . . . . . . . . . . 49

5.10 GAIN Section TDR Bias . . . . . . . . . . . . . 50

5.11 SOA Section TDR Bias . . . . . . . . . . . . . . . . 52

5.12 Wirebond Length Measurement . . . . . . . . . . . . . 55

6.1 Amplitude Modulation (AM) Experimental Test Setup . . . . . . 59

6.2 Amplitude Modulation (AM) Experimental Test Setup Photograph 60

6.3 Gain Section AM Response at $35.82 \mathrm{~mA}$ Bias . . . . . . . . . 62

6.4 Gain Section AM Response at $58.00 \mathrm{~mA}$ Bias . . . . . . . . . . 63

6.5 Gain Section AM Response at $61.90 \mathrm{~mA}$ Bias . . . . . . . . . . 64

6.6 Gain Section AM Response Summary . . . . . . . . . . . . . 65

6.7 Gain Section AM Response Relaxation Oscillation Summary . . . 66

6.8 SOA AM at $10.00 \mathrm{~mA} \ldots \ldots \ldots \ldots \ldots$

6.9 SOA Section AM Response at $100.00 \mathrm{~mA}$ Bias . . . . . . . . 68

6.10 SOA Section AM Response Summary . . . . . . . . . . . . . 69

7.1 Laser Spectrum . . . . . . . . . . . . . . . . . . . . . 72

7.2 Mach-Zehnder Interferometer Setup . . . . . . . . . . . . 75

7.3 Mach-Zehnder Interferometer Output . . . . . . . . . . . 76

7.4 Swept Sinusoid Frequency Modulation (FM) Test Setup . . . . . . 77

7.5 Phase Frequency Modulation Key Points . . . . . . . . . . 80

7.6 Phase Section FM Response at Maximum Photocurrent Intensity 82

7.7 Phase Section FM Response at Quadrature(-) . . . . . . . . . 84

7.8 Phase Section FM Response at Minimum . . . . . . . . . . . 85

7.9 Phase Section FM Response at Quadrature $(+) \ldots \ldots \ldots$. . . . 86

7.10 Phase Section FM Response: Additional Data . . . . . . . . . . 87 
7.11 Front Mirror Frequency Modulation Key Points . . . . . . . . . . 89

7.12 Front Mirror FM Response at Maximum . . . . . . . . . . . . . . 90

7.13 Front Mirror FM Response at Quadrature(-) . . . . . . . . . . . . 91

7.14 Front Mirror FM Response at Minimum . . . . . . . . . . . . . . 92

7.15 Front Mirror FM Response at Quadrature $(+)$. . . . . . . . . . . 93

7.16 Back Mirror Frequency Modulation Key Points . . . . . . . . . . 94

7.17 Back Mirror FM Response at Maximum . . . . . . . . . . . . . . 95

7.18 Back Mirror FM Response at Quadrature(-) . . . . . . . . . . . . 96

7.19 Back Mirror FM Response at Minimum . . . . . . . . . . . . . . . 97

7.20 Back Mirror FM Response at Quadrature $(+)$. . . . . . . . . . . . 98

8.1 Delayed Self-Homodyne Linewidth Test Setup . . . . . . . . . . . 102

8.2 Delayed Self-Homodyne Linewidth Autocorrelation . . . . . . . . 104

8.3 Linewidth Comparison Matrix . . . . . . . . . . . . . . . 105

8.4 Linewidth for Back Mirror Variations Only . . . . . . . . . . . . . 106

8.5 Linewidth for Front Mirror Variations Only . . . . . . . . . . . . 107

8.6 Two Sided Linewidth Measurement . . . . . . . . . . . . . . . . . 109

A.1 Laser Pinout Diagram . . . . . . . . . . . . . . . 120

B.1 Schematic Main Controller . . . . . . . . . . . . . . . 121

B.2 Schematic PID Loop . . . . . . . . . . . . . . . . . . . 122

B.3 Schematic Laser Connection . . . . . . . . . . . . . . . . . . . . 122

C.1 PCB All Layers . . . . . . . . . . . . . . . . . . . . 123

C.2 PCB Top Copper Layer . . . . . . . . . . . . . . . . . . . 123

C.3 PCB Bottom Copper Layer . . . . . . . . . . . . . . . . . . . . . 124

C.4 PCB Top Assembly Layer . . . . . . . . . . . . . . . . . . . . . . 124

E.1 AM Response $\left(S_{21}\right)$ of Varying GAIN Bias . . . . . . . . . . . . 131

E.2 AM Response $\left(S_{21}\right)$ of Varying SOA Bias . . . . . . . . . . . 134 


\section{Chapter 1}

\section{Introduction}

Medical imaging is an ever expanding field in the use of high performance optical systems to image the body. The type of imaging being discussed in this work is Optical Coherence Tomography (OCT). A popular version of OCT utilizes high speed tunable lasers. This thesis highlights performance characterization of the first 1310 nm SG-DBR lasers optimized fro OCT. Utilizing Sampled GratingDistributed Bragg Reflector (SG-DBR) lasers make it possible for Swept Source Optical Coherence Tomography (SS-OCT) to be smaller, lighter, less expensive, and higher performance than before. Previous mechanical based tunable lasers were large, cumbersome, and featured slow scan rates. This thesis is the first performance evaluation of the O-Band $1310 \mathrm{~nm}$ SG-DBR laser.

Chapter 2 begins with a brief introduction the SG-DBR applications, in particular to OCT. The fundamental advantages of SS-OCT are compared and contrasted to traditional time domain approaches.

The basic physics operating principles and brief theoretical analysis behind lasers are presented for the reader in Chapter 3. The SG-DBR's output is con- 
trolled via three frequency dependent sections and two amplitude sections to achieve wavelength tuning. An illustration of the performance metrics relating to OCT is also presented.

The testing fixture designed for all experiments and to serve as a general purpose breakout board for packaged lasers is described in detail in Chapter 4 . The RF design considerations of the PCB as well as the implementation of a full analog PID controlled Thermal Electric Cooler (TEC) control circuit is detailed. The testing fixture allows for rapid deployment of additional lasers for testing.

Chapter 5 begins to deliver the experimental data and analysis into the performance metrics of the laser. It begins with equivalent circuit models for each port of the laser, and how each respective port responds to varying bias current. Next, in Chapter 6 Amplitude Modulation is performed on each of the Gain and Semiconductor Optical Amplifier (SOA) sections to observe the maximum operating modulation frequency, which limits the scan repetition rate for OCT. Frequency modulation is performed in Chapter 7 on the three frequency dependent sections of the Front Mirror (FM), Back Mirror (BM), and Phase Medium (PM) to examine each sections amplitude and frequency dependence. Finally, in Chapter 8 the optical linewidth is measured and quantified for a myriad of bias currents. For each section theory is presented along with experimental test setup arrangements.

Lastly, Chapter 9 presents a detailed summary of the various optical and electrical measurement results investigated. Considerations for future research and investigation are detailed. 


\section{Chapter 2}

\section{Application Background}

Optical Coherence Tomography (OCT) is an exciting and relatively new medical imaging technique, first developed at MIT in 1991 [1]. From initially starting out primarily in $\mathrm{R} \& \mathrm{D}$ applications, it has since transformed into use in hundreds of clinical procedures and even industrial applications. OCT also has the ability to not only image in vivo, for example high resolution endoscopy or arterial wall mapping, but even ex vivo, in the case of skin and retinal imaging.

The basic premise behind traditional OCT is quite similar to ultrasound, however utilizing infrared light instead of sound. To understand the operating principles behind OCT, first imagine pulses being sent into a tissue sample, as with ultrasound. The instrument then listens for a series of echoes backscatting from the tissue at different depths. If the instrument is fixed in a single position

this will produce a one-dimensional depth profile, known as an A-scan [2]. Now consider scanning the instrument laterally across the tissue sample to construct a 2D mapping or cross section of a tissue indicating depth verses sample scan.

OCT holds a primary advantage over ultrasound in that the imaging resolu- 


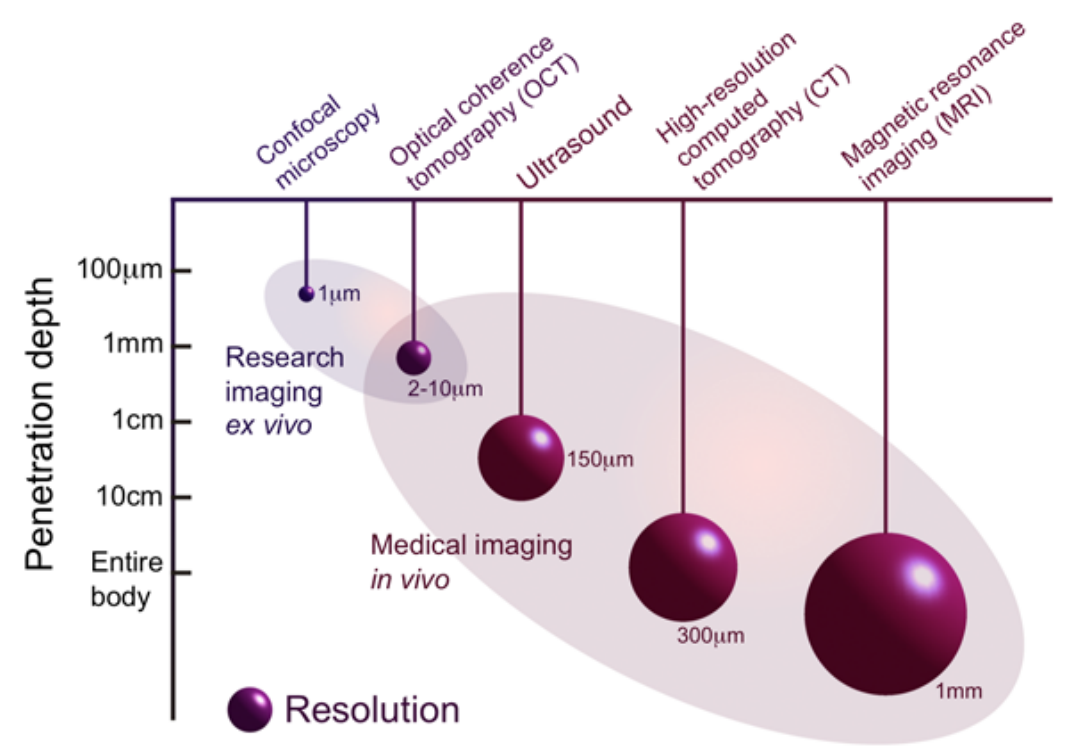

Figure 2.1: Medical Imaging Technique Comparison. Penetration depth is compared to various techniques along with respective resolution and preferred application. OCT resolution is 2-10 $\mu \mathrm{m}$ with penetration of several $\mathrm{mm}$ [3].

tion of 2-10 $\mu m$ is greatly enhanced, while still exhibiting moderate tissue penetration depth of approximately $4 \mathrm{~mm}$ [3]. Magnetic Resonance Imaging (MRI) on the other hand enables full body imaging, at the cost reduced resolution (1 $\mathrm{mm}$ ), and increased imaging time for the patient. A comparison of OCT imaging to various alternative medical imaging procedures is shown in figure 2.1. The initial instrument cost is also significantly less, on the order of several tens of thousands of dollars compared to several million for a modern MRI. Compact size and portability allow for potential mobile imaging applications.

\subsection{OCT Approaches}

In the ultrasound analogy example given previously, it described the echoes that are reflected back to the source, and time domain analysis. A slight mod- 
ification must be made to the original analogy when dealing with light instead of sound. Instead, the use of an interferometer can be implemented to essentially downmix the high frequency optical components into a range detectable by photodetectors. Figure 2.2a illustrates the time domain approach with a simple Michelson interferometer. This type of conventional approach is known as Time Domain OCT (TD-OCT). The reference path is scanned via an optical delay line (ODL), acting as a movable mirror, for constructive interference to occur at the photodetector [2]. Therefore, for a distance of $x_{\text {depth }}$ desired, the reference arm must similarly be displaced.

Moving from the time domain approach into spectral based solutions for OCT leads to two primary candidates: Spectral Domain (SD) and Source Swept (SS), collectively known as Fourier Domain for OCT. For both solutions, the moving reference mirror is removed and replaced with a static mirror giving a fixed reference path. The detector relies upon backscattered light, and deconstructs the individual frequency components. In the case of SD-OCT, the same low coherent broadband light source is used to couple light into the interferometer, however the detector has been replaced with a low-loss spectrometer. This spectrometer detects the spectral variation from the interferogram generated by the Michelson interferometer through a diffraction grating onto a CCD array [4].

The final method of SS-OCT, of which the SG-DBR is concerned, utilizes a repetitive and continuous wavelength sweep of highly coherent light. This method's end detection of a single photodetector is much simpler than the CCD array of the SD-OCT, however it gains in complexity of tuning the SG-DBR to provide a consistent linear frequency ramp. Just as before, individual scans result in generating depth mapping, while lateral scanning provides a cross sectional view of tissue. A comparison between the time domain and two spectral domain 


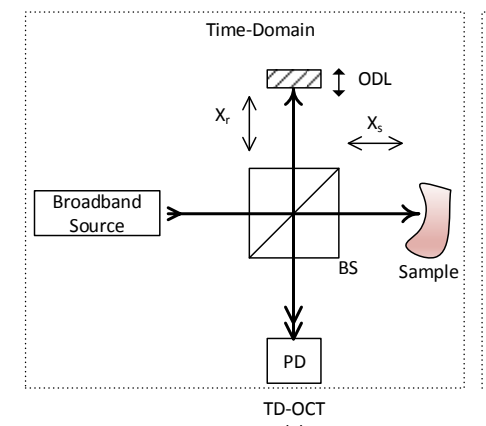

(a)

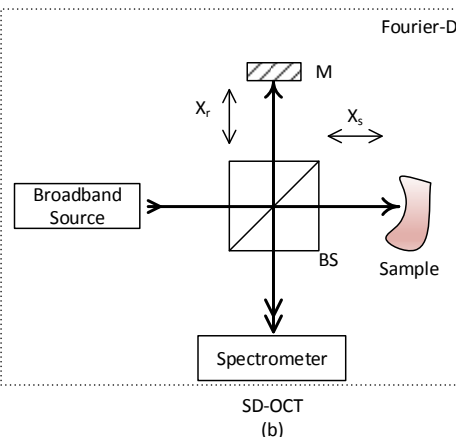

(b)

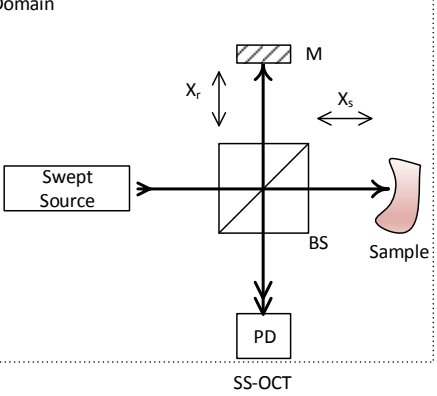

Figure 2.2: Time and Fourier Domain OCT Comparison. (a) Time Domain OCT with a broadband light source. The ODL controls the imaging depth in the sample. Interference signal is fed into a simple photodetector. Two types of Fourier Domain include (b) Spectral Domain OCT (SD-OCT) with a broadband source and spectrometer for analysis. In SD-OCT a spectrometer analyzes the frequency components of the reflected light from the broadband source. (c) In Source Swept OCT (SS-OCT) the laser is swept in frequency causing interference at various depths in the sample observed on the photodetector. There are no mechanical or moving parts in this approach. (M, fixed mirror; ODL, optical delay line; PD, photodetector; BS, beamsplitter.)

OCT approaches are presented in table 2.1. The larger the tuning range for a SG-DBR laser, the higher the depth resolution and the wider variety of tissue densities can be imaged. Also, although intuitive, the higher the scanning rate the faster a measurement can be taken.

Experimental test results from a SS-OCT implementation, however with a $1550 \mathrm{~nm}$ laser, are shown in figure 2.3. The left images show a series of B-scans (several A-scans panned laterally and stitched) of a skin tissue sample at various A-scan rates of $36.8,109$, and $155.8 \mathrm{KHz}$. The right most side corresponds to a 16 frame averaged B-scan image of the same tissue. Figure 2.4 adds not only lateral scanning but longitudinal scanning to produce a 3D depth mapping of human tissue, in vivo. The particular sample illustrated is a $5 \times 5 \times 5 \mathrm{~mm}^{3}$ 
Table 2.1: Comparison Between TD-OCT, SD-OCT, and SS-OCT

\begin{tabular}{|l||l|l|l|}
\hline & TD-OCT & SD-OCT & SS-OCT \\
\hline \hline Source & Broadband & Broadband & Swept Coherent \\
\hline Detector & PD & Spectrometer & PD \\
\hline Ref. Mirror & Movable & Fixed & Fixed \\
\hline Post Processing & Simple & Complex & Complex \\
\hline Mechanical Modulation & Yes & No & No \\
\hline SNR & Medium & High & High \\
\hline OCT System & Medium & Simple & Complex \\
\hline Light Source & Simple & Simple & Complex \\
\hline
\end{tabular}

volume, and was acquired in less than 2.4 seconds. 
single B-scan
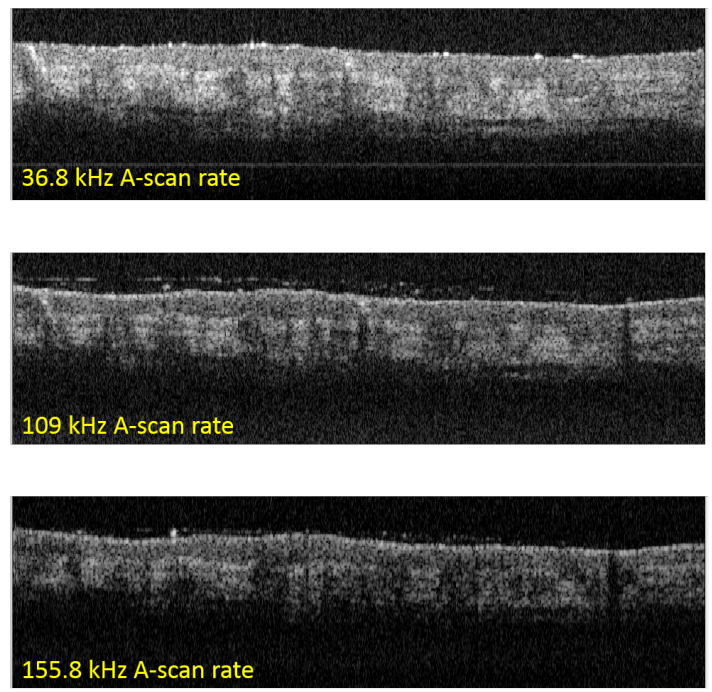

averaged frame (16 B-scans)
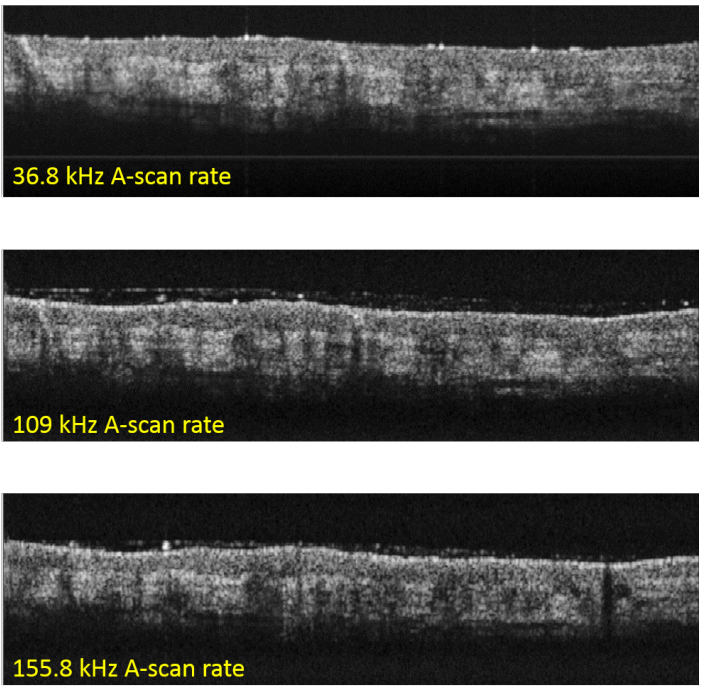

Figure 2.3: OCT example obtained from $1550 \mathrm{~nm}$ SG-DBR laser of human skin, at $\sim 2 \mathrm{~mW}$ power. Single frame captures vs. averaged frames at various sweep rates. Single captures in (a)-(c) performed at $36.9 \mathrm{KHz}, 109 \mathrm{KHz}$, and $155.8 \mathrm{KHz}$ respectively. 16 frame averaged captures in (e)-(f), with the same sweep rates of (a)-(c). Imaging area was $5 \times 1.7 \mathrm{~mm}^{2}$. Noticed for the slower repetition rate in (a) and (e) images appear brighter due to increased illumination time of the sample. [5] 


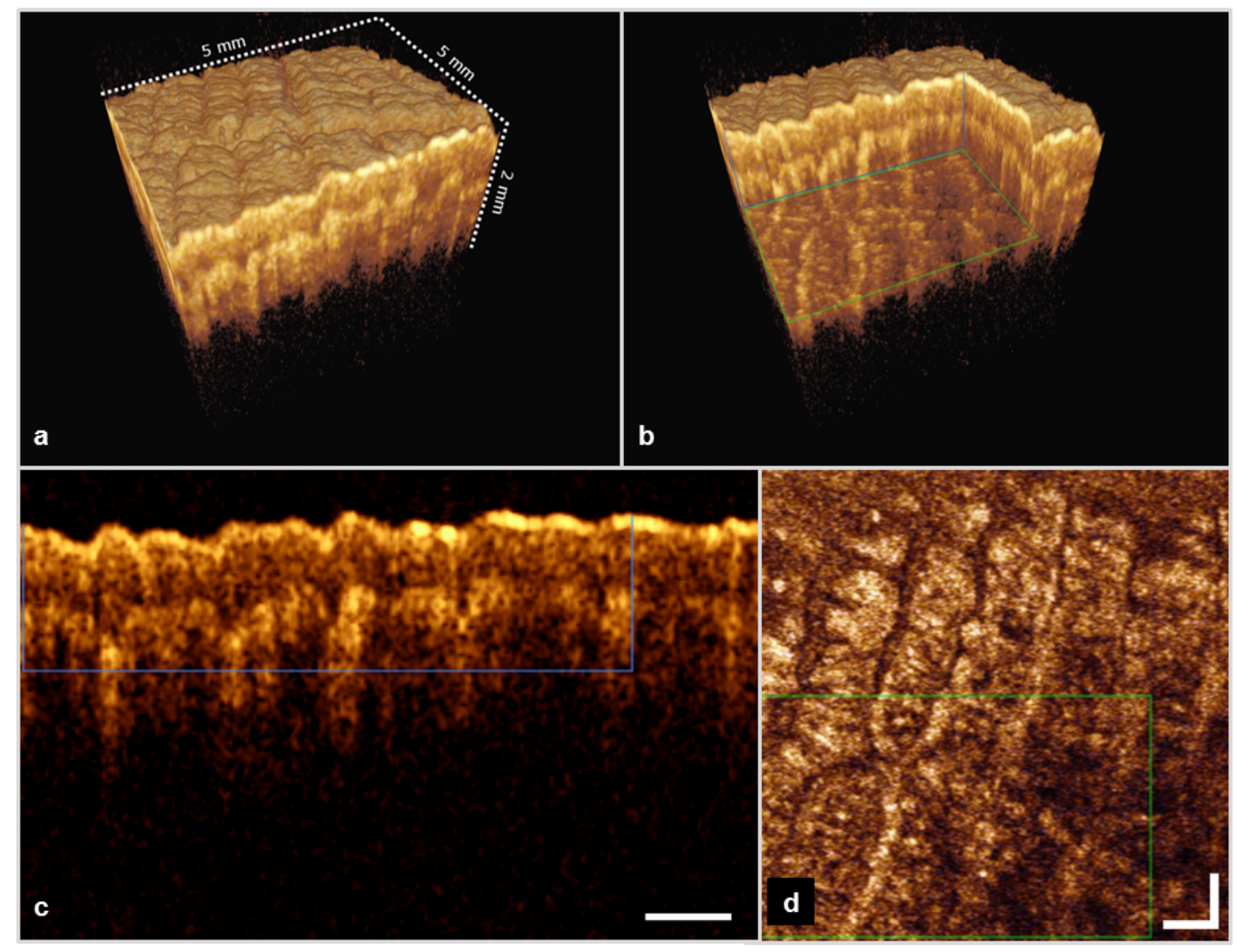

Figure 2.4: 3D OCT Image of Skin from $1550 \mathrm{~nm}$ laser. (a) 3D reconstruction of data; (b) $3 \mathrm{D}$ reconstruction with a portion removed to reveal the internal structure of the tissue; (c) cross sectional slice from the complete $3 \mathrm{D}$ dataset. The portion is shown in the blue rectnagle in (b); (d) en-face view from the 3D dataset. The rectangle corresponds to the horizontal green outline in (b). Captured volume of $5 \times 5 \times 5$ $\times \mathrm{mm}^{3}$. Actual images cropped in $\mathrm{z}$ dimension to $2 \mathrm{~mm}$ to present the useful area. All scale bars represent $0.50 \mathrm{~mm}$. [5] 


\section{Chapter 3}

\section{SG-DBR Tunable Laser Background}

\subsection{Laser Physics}

The laser has become ubiquitous in everyday life since its discovery by Townes and Schawlow in a paper entitled Infrared and Optical Masers, published in Physics Review in 1958 [6]. Lasers have become such commonplace in society today that it is interesting to note the term laser is not actually a word as traditionally thought, but rather an acronym for Light Amplification by Stimulated Emission of Radiation [7]. While it is not suitable for general purpose illumination, applications that require a very narrow frequency distribution, high intensity, high collimation, or ultra fast pulses are ideal uses for lasers. For example, consider a traditional light source of a tungsten bulb. A flow of current causes heating and thus illumination to radiate outward in all directions light that is incoherent, containing randomly distributed phases. Now consider con- 
centrating all of the radiating light into a single direction, and amplifying it that fundamentally makes a laser.

This essentially forms the basis of an optical oscillator, as shown in figure 3.1. There is a resonant optical amplifier, known as the active medium, when without feedback will simply amplify small amounts of noise present in the medium, provided they are within the bandwidth of the amplifier. The start of the oscillation truly begins with the addition of feedback elements, in the case of two mirrors as the resonator elements. A wave in between the mirrors, traveling orthogonal, will bounce back and forth, each time being increasingly amplified by the active medium. If one of the mirrors is partially transmissive, or in other terms partially transparent, a portion of the beam can be extracted out.

In order for an output to be obtained two basic conditions must be met for lasing to occur. A certain gain condition must be satisfied known as the laser threshold. Namely, oscillation will only commence when the gain of the active medium exceeds any losses internally [8]. The second basic oscillatory condition is that the phase shift for a light wave be a multiple of $2 \pi$ for a round trip inside the resonator. From this it is apparent that the effective distance between the mirrors in turn defines the primary resonant frequency of the oscillator! If the distances can be adjusted, this forms the basis of a tunable laser as will be described later in section 3.3.

Understanding the laser operation from a simple schematic is adequate at first, however to prepare for other sections, coverage of the electromagnetic phenomena involved in lasers is essential. These are spontaneous and stimulated emission, and absorption, which are constantly occurring during laser operation. Consider an atom or a molecule to have two energy levels, denoted 1 and 2 with energies $E_{1}<E_{2}$, respectively in energy level diagram in figure 3.2. Spontaneous emission 


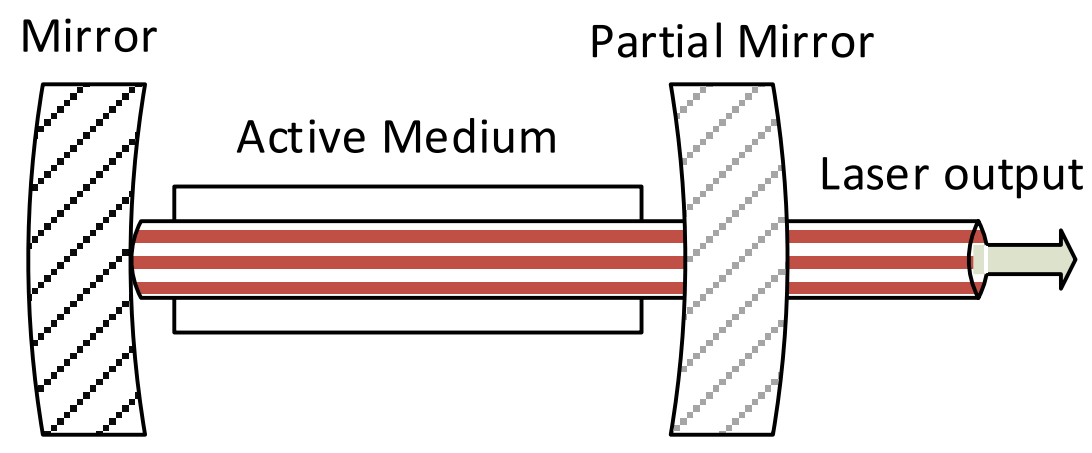

Figure 3.1: Generic Laser Schematic consists of an optical amplifier between a pair of mirrors, one of which is partially transmissive.

in part (a) is classified when an atom at a higher level decays down to a lower level, thus radiating a photon,

$$
h \nu=E_{2}-E_{1}
$$

where $h$ is Planck's constant, and $\nu$ is the frequency of the radiated wave, and $E$ are the energies. The second essential properly of laser is stimulated emission, shown in part (b) of figure 3.2. Assume an atom begins in the higher energy level 2 , and there is an electromagnetic wave with a frequency $\nu$ incoming. Because they are are the same frequency, there is a high probability for a transition to occur from $2 \rightarrow 1$, thus releasing a second photon [8]. The important difference to note is that with spontaneous emission the radiated photon has no phase correlation with other atoms, and can radiate in any direction. For stimulated emission, because the transition is induced by an incident wave, the released atom will be in phase with the original wave, with the same polarization, and therefore 


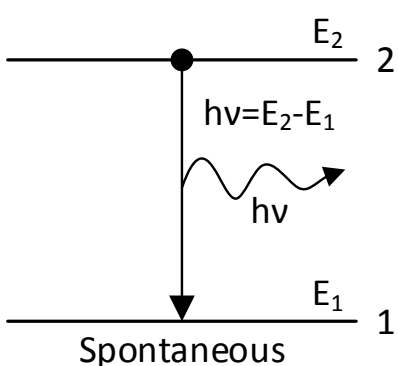

(a)

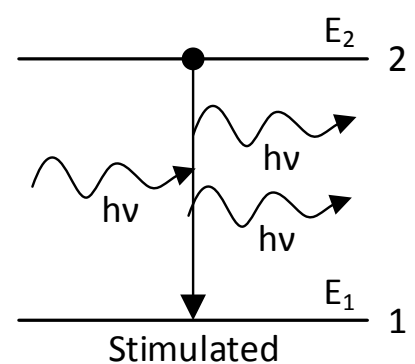

(b)

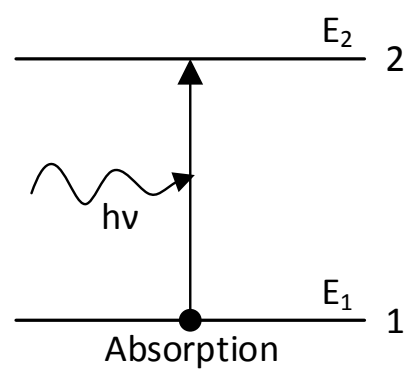

(c)

Figure 3.2: Laser Emission Processes consist of: (a) spontaneous emission, (b) stimulated emission, and (c) absorption, shown in a simplified 2 level energy diagram.

direction - essentially a clone photon. Lastly, absorption in part (c) is the third primary transitional phase in which an incident wave is absorbed, causing an increase in the energy level of the atom. It is the combination of spontaneous and stimulated emission that forms the foundation for laser operation.

A final key element in the understanding of lasers is the creation of a population inversion, which ensures a greater probability of stimulated emission over absorption. A population inversion simply states a higher percentage of atoms exist a higher energy state than the ground state in a multi level energy system [9]. Without this population inversion present, a system would eventually balance out with equally distributed energy in both high and low levels.

\subsection{Semiconductor Laser Diode}

A semiconductor type of laser is not much different than a standard p-n junction type diode. Previously we discussed discrete energy diagram for each atom, however when dealing with semiconductors we must consider the entire 


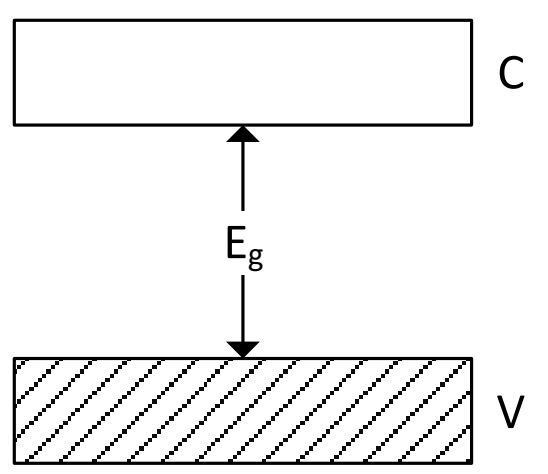

(a)

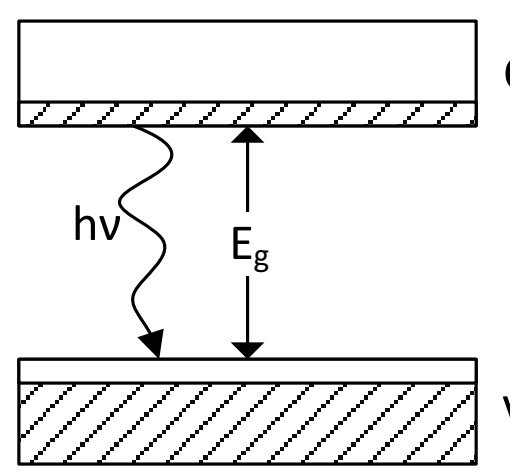

(b)

Figure 3.3: Semiconductor Laser Energy Diagram. Electrons are indicated by shaded region. (a) The semiconductor valance band, $V$, is separated from the conduction band, $C$, by the band gap energy $E_{G}$. (b) Stimulated emission process.

crystal's energy bands. Again, consider an energy diagram as shown in figure 3.3 of a semiconductor laser. In figure 3.3a the valance band, $V$, and conduction band $C$ are separated by the band gap energy $E_{G}$, with the valance band completely full of electrons indicated by the shading. Assume that electrons from the fully populated valance band are somehow raised into the conduction band at a higher energy. Soon after, the top of the valance band will be vacant from electrons leaving holes in the upper layer. Likewise in the conduction band the electrons will settle to the lowest levels as indicated in figure 3.3b. There is now a population inversion between the two bands. The electrons in the top conduction will fall down to meet with the holes in the valence layer, producing stimulated emission of photons $h \nu$, and laser oscillation if inside of a resonator with the threshold conditions satisfied.

For this process of electron and hole recombination to sustain, an external en- 
ergy source must be used to excite the pairs to continue to recombine and radiate. For semiconductors simply forward biasing the device will cause recombination radiation, known as injection electroluminescence as for a simple Light Emitting Diode (LED) [9]. Once the aforementioned threshold is reached, the number of electron and hole recombination will large enough to provide the population inversion, where the stimulated emission will be greater than the absorption.

With the semiconductor laser it may not immediately be apparent how light propagates out into the real world. From before consider a p-n type device in forward bias, with current flowing into the top anode (p-type). At the junction between the two types there exists the depletion region. For a homojunction, or a device with only a single semiconductor present this region is where recombination occurs. Another type is known as a heterojunction, in which an additional semiconductor is added between the primary $\mathrm{p}$ and $\mathrm{n}$ types, creating a region known as the active layer. This creates a cladding layer surrounding the inner active layer as shown in figure 3.4, which have a higher band gap and a lower refractive index than the active. The difference in band gap forces electrons and holes to combine within the active region, along with the step refractive index creating a dielectric waveguide. This optical waveguide channels the photons into a particular mode, illustrated in the bottom of the figure. To produce the mirror like effect for the resonator, the facets parallel to are cleaved, where the orthogonal surfaces are roughened. 




Figure 3.4: Double-Heterostructure Semiconductor Laser Profile View. The width of an Active Layer sandwiched between $\mathrm{p}$ and $\mathbf{n}$ type plotted for depth into the device. A step in index of refraction creates an optical waveguide, guiding a dominant mode to propagate. Figure modeled after Ref. [6]. 


\subsection{SG-DBR Laser Structure}

Now that the basics of semiconductor diodes have been introduced, operation of the Sampled-Grating Distributed Bragg Reflector (SG-DBR) of this thesis can be discussed. The simple semiconductor laser introduced previously did not provide the ability to be tuned, or change the wavelength output, nor did it necessarily produce a single longitudinal mode. The relative mirror positions were fixed for the semiconductor and gas lasers, preventing the ability to tune the wavelength output. Regarding the topic of the single longitudinal mode, to achieve this result in semiconductor lasers a Distributed Feedback (DFB) can be constructed. This essentially forms a corrugated optical grating above the active layer, which in turn behaves like a wavelength selective filter to only allow certain modes [10]. Think of the grating as having its own set of resonant modes it supports as well as the mirrors having their own respective set. The single longitudinal mode will be whatever mode lies in common with both resonators. The particular wavelength of the periodic grating is derived from Bragg's Law,

$$
\Lambda=\frac{m \lambda_{0}}{2 n_{e f f}}
$$

where $\Lambda$ is known as the grating period, $\lambda_{0}$ is the freespace wavelength, $m$ is the integer order of the diffraction, and $n_{e f f}$ is the effective refractive index of the cavity. This wavelength selectivity makes it ideal for ideal for telecommunications applications and others requiring narrow linewidths compared to standard laser diodes.

For SS-OCT applications, as well as Wavelength Division Multiplexing (WDM) in telecommunications, it is essential to operate and tune to several discrete wavelengths. The DFB laser already has the ability to be tuned by modifying the 
temperature or drive current [10]. However the tuning range is limited to approximately $10^{-2} \mathrm{~nm} / \mathrm{mA}$ for DFBs; not nearly wide enough for tuning within an entire optical band. For a more practical tunable semiconductor laser, the Distributed Bragg Reflector (DBR) allows for much wider ranges. A standard DBR contains three primary regions: gain, phase, and the Bragg mirror. Individual currents are applied to each section, with the gain current $I_{G}$ controlling the output power of the laser. The phase current $I_{\phi}$ controls the phase of the feedback from the Bragg mirror section. The Bragg current $I_{B}$ controls the Bragg wavelength, as in equation 3.2, causing the effective index of refraction to change, thus leading to a changing wavelength.

The SG-DBR consists of a total of five waveguide sections: gain section, front mirror, back mirror, phase section, and finally a Semiconductor Optical Amplifier (SOA) as illustrated in the top view in figure 3.5. The gain section is situated in between the two primary mirrors, and contains the active layer inside the waveguide. The front and back mirror sections are very similar to each other, however the front mirror is much shorter, each created by a series of sampled gratings [11]. Sampled gratings refer to the periodic etching away in the passive layer located above the waveguide structure. The phase section is simply a passive delay section without an active region or gratings. The SOA section is very similar to the gain section before, with the exception that it is located outside the front mirror and acts as an additional inline amplifier to boost output optical power.

When injected current enters the three frequency dependent sections of the laser (front, back, and phase), the carriers induce a change in refractive index, thereby tuning the wavelength and overall mode spectrum of the cavity. The effect of sampling the passive region above in the front and back section causes 


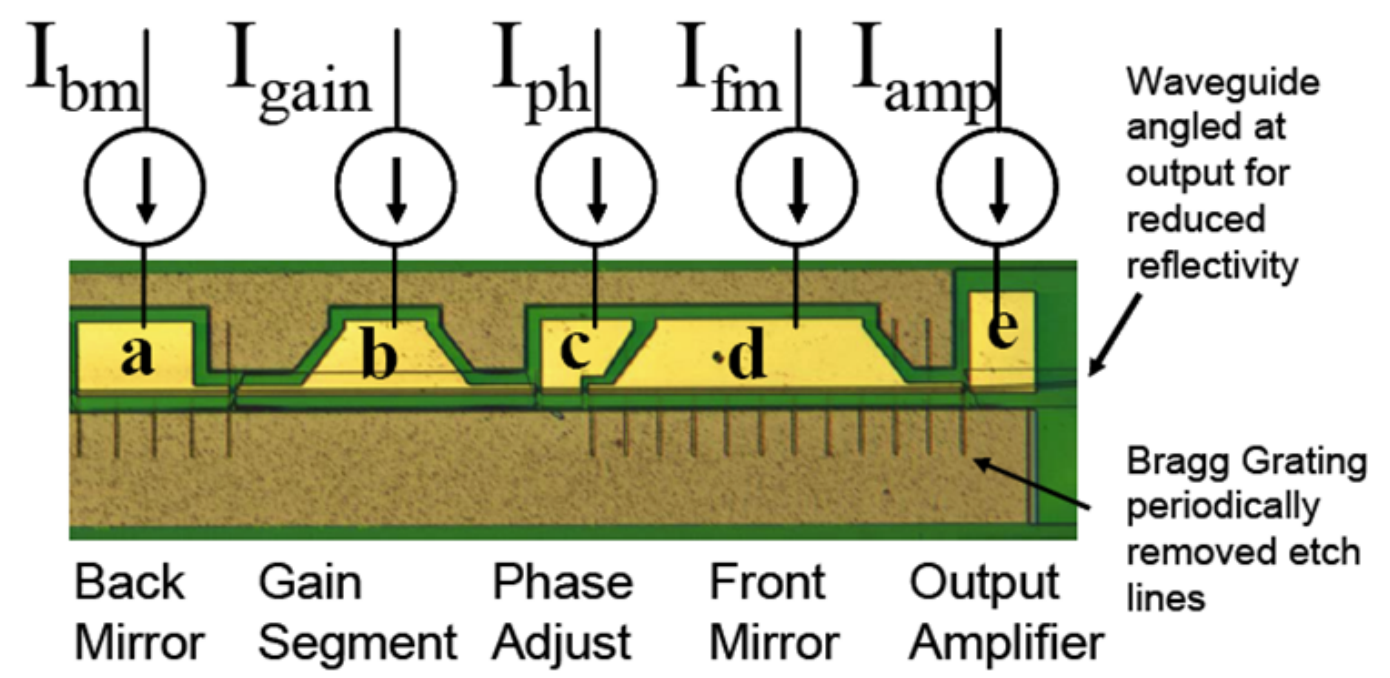

Figure 3.5: Structure of the SG-DBR laser, top down illustration. Five sections control the laser's output power and frequency. The gain section (b) is located between the front (d) and back (a) mirrors, containing an active layer in the waveguide. Front and back mirrors are similar, each containing sampled gratings above the waveguide structure. The phase (c) is a passive delay section located between the two primary front and back mirrors for fine frequency tuning. The SOA (e) provides additional amplification of the output optical power. Illustration not to scale. [12]

a periodic comb of reflectance peaks for the sampled DBR compared to only a single peak in a standard DBR. Now consider if each of the front and back mirror have a different periodic comb spacing, then the lasing wavelength inside of the waveguide will be found when the front and back mirror relativity peaks align with maximal overlap, as shown in figure 3.6. The spacing between the Bragg grating sets, denoted as $L_{m}$, where $m=1,2$ for the front and back mirrors, respectively, relates to the wavelength difference, $\Delta \lambda_{m}$, according to equation 3.3, indicating an inverse relationship between the $L$ and $\Delta \lambda_{m}$.

$$
\Delta \lambda_{m}=\frac{\lambda^{2}}{2 n L_{m}}
$$


It is important to note that current for the two mirror sections will equally tune all of the reflectance peaks at the same time. Current into the phase on the other hand will tune the individual cavity modes simultaneously by setting the roundtrip phase in $n \times 360^{\circ}$ multiples for constructive interference. This allows for high precision and continuous tuning of wavelength, compared to the coarse tuning ability provided by solely tuning the primary mirrors. It is evident that for a continuous tuning within the band, which is essential for OCT, a combination of all three frequency dependent sections must be tuned in concert.

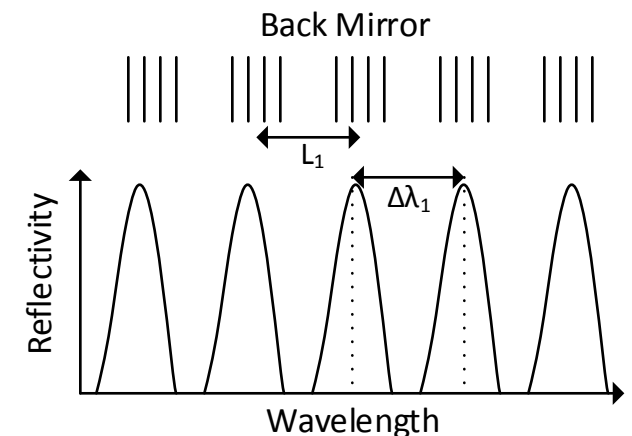

(a)

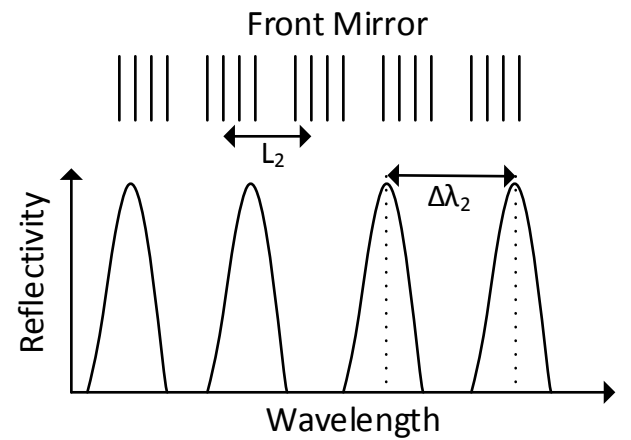

(b)

Figure 3.6: SG-DBR Reflectivity Structure. Bragg reflectors for front (a) and back (b) mirrors. Spacing between gratings denoted $L_{1}$ and $L_{2}$ for front and back, respectively, relate to wavelength changes of $\Delta \lambda_{1}$ and $\Delta \lambda_{2}$. Figure modeled after Ref. [13]. 


\subsection{Single Mirror Tuning}

For wavelength tuning, the simplest manual form is adjusting the injection current into a single mirror at a time. For the front mirror and back mirror sections, individual tuning allows for approximately $4 \mathrm{~nm}$ increments before a mode hop occurs. Fixing a particular mirror and only adjusting the opposite mirror allows the reflectivity peaks to transition into new modes gradually. In figure 3.7, the front mirror is fixed while the back current is adjusted. In part (a), a single primary mode, designated mode 1 is dominant; however as current is injected into the back mirror in parts $(\mathrm{b})-(\mathrm{d})$, secondary modes begin to become stronger. Finally in part (e), mode 2 becomes the primary dominant mode. Part (f) of 3.7 demonstrates at least 4 prominent modes in the cavity resonating. This particular case of figure $3.7 \mathrm{f}$ is one that should be avoided, as for maximum signal integrity a single longitudinal mode (SLM) is desired.

As mentioned previously in figure 3.6, the primary mirror sections contain Bragg Gratings, which in turn form reflectivity peaks. Figure 3.8 conceptually illustrates adjusting the front mirror bias (a) and (d) while keeping back mirror bias fixed in (b) and (e). When the peaks align spatially for the two mirror sections, as indicated by vertical lines in the figure, it results in a particular wavelength output as in parts (c) and (f). Recall back to figure $3.7 \mathrm{f}$ with multiple longitudinal modes propagating. This characteristic can be explained from observing the repetitive reflectivity peaks, where several appear to be partially aligning instead of the ideal case of a single perfect alignment, causing multiple output peaks to be present. 
(a)

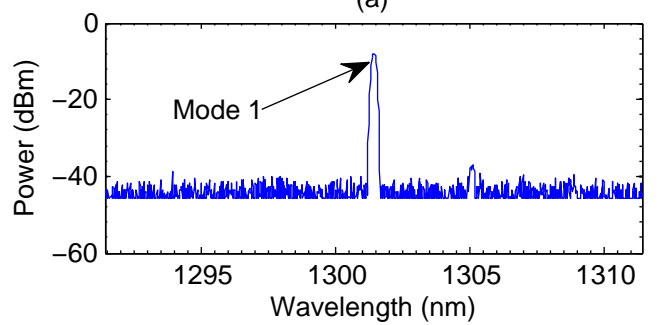

(c)

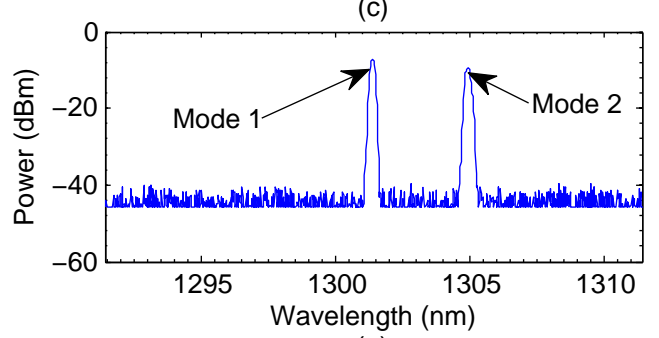

(e)



(b)

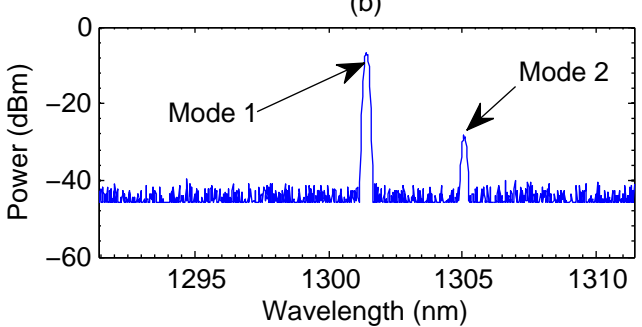

(d)

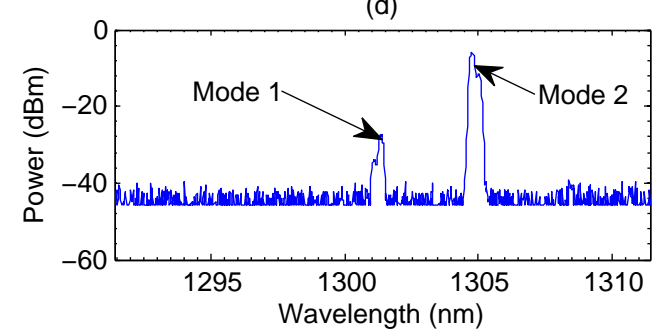

$(\mathrm{f})$

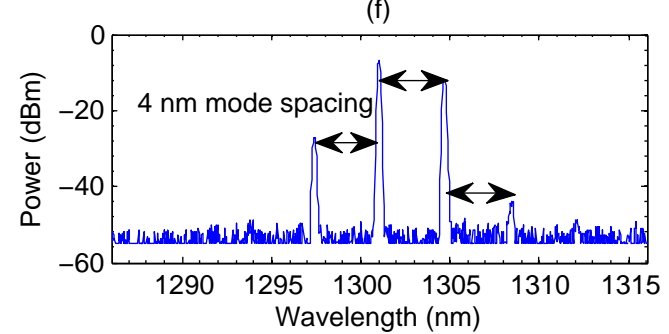

Figure 3.7: SG-DBR Single Mirror Tuning Example. Back mirror biasing is varied with fixed phase, front, gain, and SOA currents. (a) Mode 1 is dominant. (b) Mode 2 growing in amplitude. (c) Both modes are nearly equal in power. (d) Mode 2 is greater than 1 . (e) Mode 2 dominates over Mode 1. (f) Multiple secondary modes exhibiting 4 $\mathrm{nm}$ spacing between adjacent modes.

\subsection{Dual Mirror Tuning}

Previously with only single mirror tuning, increments of approximately $4 \mathrm{~nm}$ were possible before mode hopping. Recall that the Bragg spacing of the back and front mirror, $L_{1}$ and $L_{2}$ respectively, are slightly different between mirrors. This allows for more instances of overlap and therefore more modes when both sections are tuned simultaneously [13]. As indicated in figure 3.9, when both the front and back mirror are injected with current, the mode hop is greatly reduced 

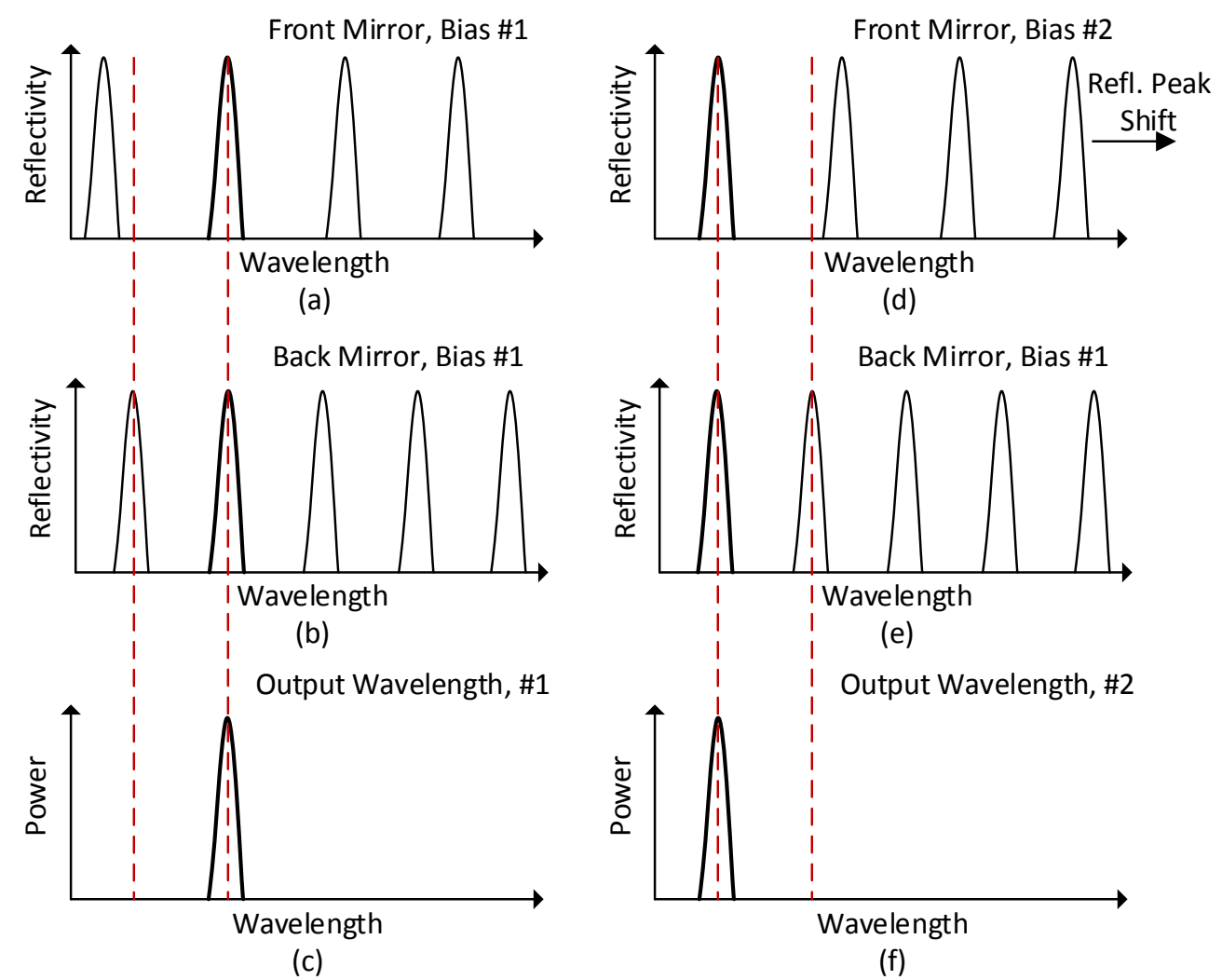

(d)
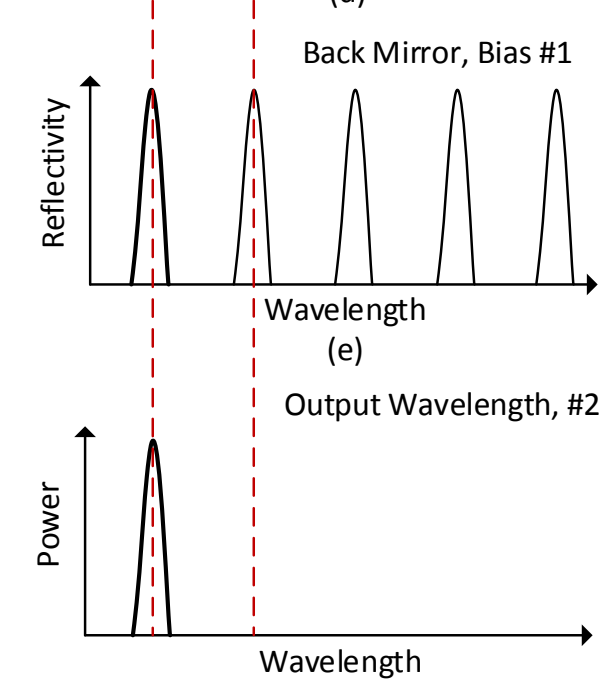

(f)

Figure 3.8: SG-DBR Single Mirror Tuning Conceptual Illustration. Back Mirror bias is fixed in (b) and (e). Front Mirror biasing from (a) yield optical output in (c), likewise changing Front Mirror to (d) produces output in (f). Red dashed lines indicate reflectivity peaks alignment of back mirror. Figure modeled after Ref. [13].

to approximately $0.3 \mathrm{~nm}[14]$.

\subsection{Phase Section Tuning}

The transition from single to dual tuning provided improvements from $4 \mathrm{~nm}$ to approximately $0.3 \mathrm{~nm}$ tuning resolution. However for successful SS-OCT applications, a fully continuous sweep of frequency is necessary without the coarse 

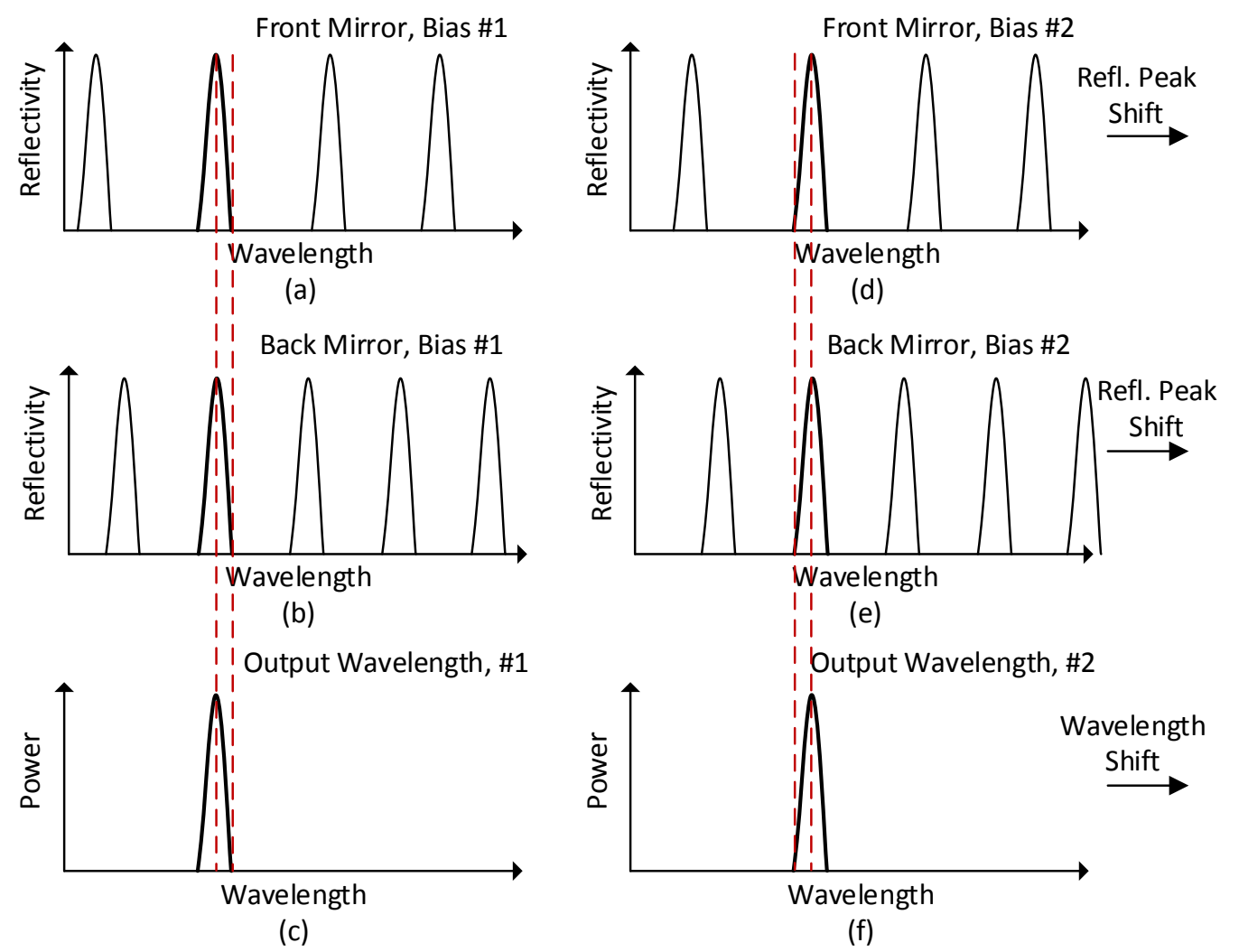

Figure 3.9: SG-DBR Dual Mirror Tuning Conceptual Illustration. Mode hop is greatly reduced to $0.3 \mathrm{~nm}$ with simultaneous dual tuning. Red dashed lines indicate reflectivity peaks alignment. (c) shows optical output of bias case (a) and (b), similarly for output (f). Figure modeled after Ref. [13].

mode hops. The addition of a third tuning element, the phase section, allows for even finer continuous range of approximately $0.2 \mathrm{~nm}[14]$. When all three elements are controlled in harmony a fully continuous tuning range is possible without any mode hopping. An example of only phase based tuning is presented in figure 3.10. In this experiment the Optical Spectrum Analyzer (OSA) is configured to run in max hold mode. The front and back mirror are fixed to a constant bias while the phase section is slowly tuned from high to low currents until a 
mode hop occurred. The output wavelength increases from left to right, inversely related to the decreasing phase current. The oscillations on top of the peak is due to the frequency of the particular interferometer connected during an alternate test, and if connected directly would not be present. Notice the approximate tuning range of $0.2 \mathrm{~nm}$, as indicated by the tune start and stop markers.



Figure 3.10: SG-DBR Phase Tuning Example. Phase is tuned from high to low current, with back and front mirror bias fixed. Max hold amplitude on OSA to observe wavelength sweep. 


\subsection{Motivations for $\mathrm{OCT}$}

Several key questions must be asked when investigating a new laser for OCT. Recall from section 2.1 for SS-OCT to be viable, the source wavelength must be rapidly swept. The swept wavelength then is split into an interferometer, in this case a Mach-Zehnder as indicated by the beam splitter in figure 3.11. The light reflects off the sample and recombines with the reference mirror path onto the photdetector. The performance of a such an OCT system relies upon the performance of the SG-DBR, and in turn measuring and determining these limits of operation.

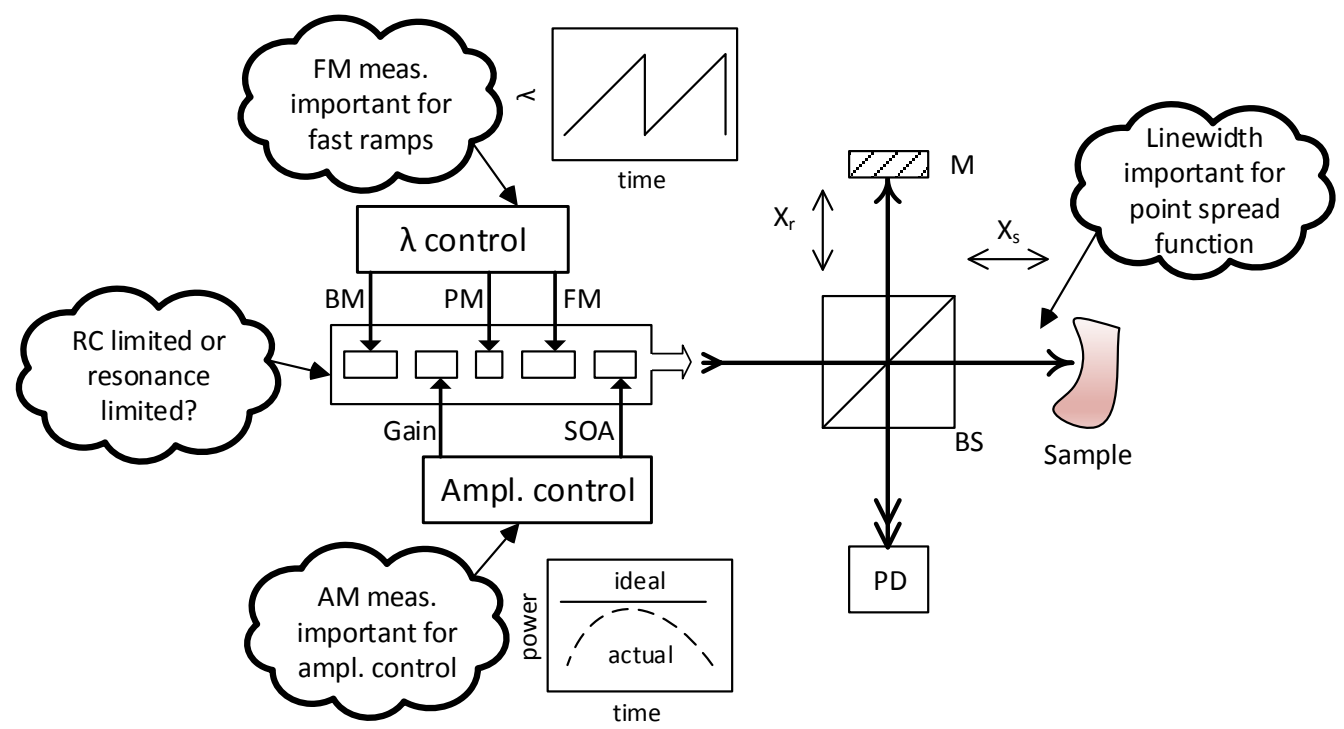

Figure 3.11: Measurement Motivations for SS-OCT. Equivalent circuit models of the frequency and amplitude control sections determine if the device is RC parasitic limited or resonance limited. Frequency Modulation measurements are critical for determining how fast the frequency ramps can be for SS-OCT. Amplitude control is necessary to maintain constant power output levels while tuning. Linewidth is a measure of the spectral purity of the laser source.

As indicated in figure 3.11 by the cloud symbols, the essential questions investigated in this thesis are presented. The diodes inside the laser and the packaging 
determine the $\mathrm{RC}$ parasitics of the device. Creating equivalent circuit models yields an understanding if the device is $\mathrm{RC}$ limited or relaxation limited at particular bias currents.

For frequency control, frequency modulation tests performed determine the bandwidth of each of the three frequency tuning elements. A constant linear ramp of frequency with respect to time is essential for SS-OCT, the repetition rate of which is determined by how fast each individual section can be modulated.

While the frequency is tuned constantly, the amplitude of the primary mode does not remain constant. Therefore in OCT operation the two amplifier sections are adjusted simultaneously to compensate for power level fluctuations and maintain as constant as possible output power. The limits of which are determined via amplitude modulation tests on the gain and SOA sections.

Finally, as an indication of the spectral purity of the output optical source, the linewidth is measured. The cleaner and tighter the linewidth, the higher the performance and greater resolution of the SS-OCT. Longer coherence length (smaller linewidth) enables the OCT probe to be positioned further away from the sample. 


\section{Chapter 4}

\section{Testing Fixture}

The Insight Photonics SG-DBR is encased in a gold plated 14 pin butterfly

package, the pinout is shown in appendix A. To interface with the laser, a testing fixture was created to act as a breakout and add additional functionality such as an integrated Thermal Electric Cooler (TEC) controller. Since multiple lasers were to be tested, an efficient and compact test fixture needed to be created to facilitate ease of construction and enhanced reliability compared to individually prototyping a breakout board from bare copper clad.

\subsection{PCB Design}

The PCB design chosen is a compact $4 \times 5$ " two layer FR4 PCB, fabricated from Advanced PCB. An important aspect of the Front, Back, Phase, Gain, and SOA sections is that they require high speed ramp input waveforms. Therefore specialized RF layout considerations must be taken into account when designing a PCB interface to reduce loss, distortion, and maintain signal integrity. 


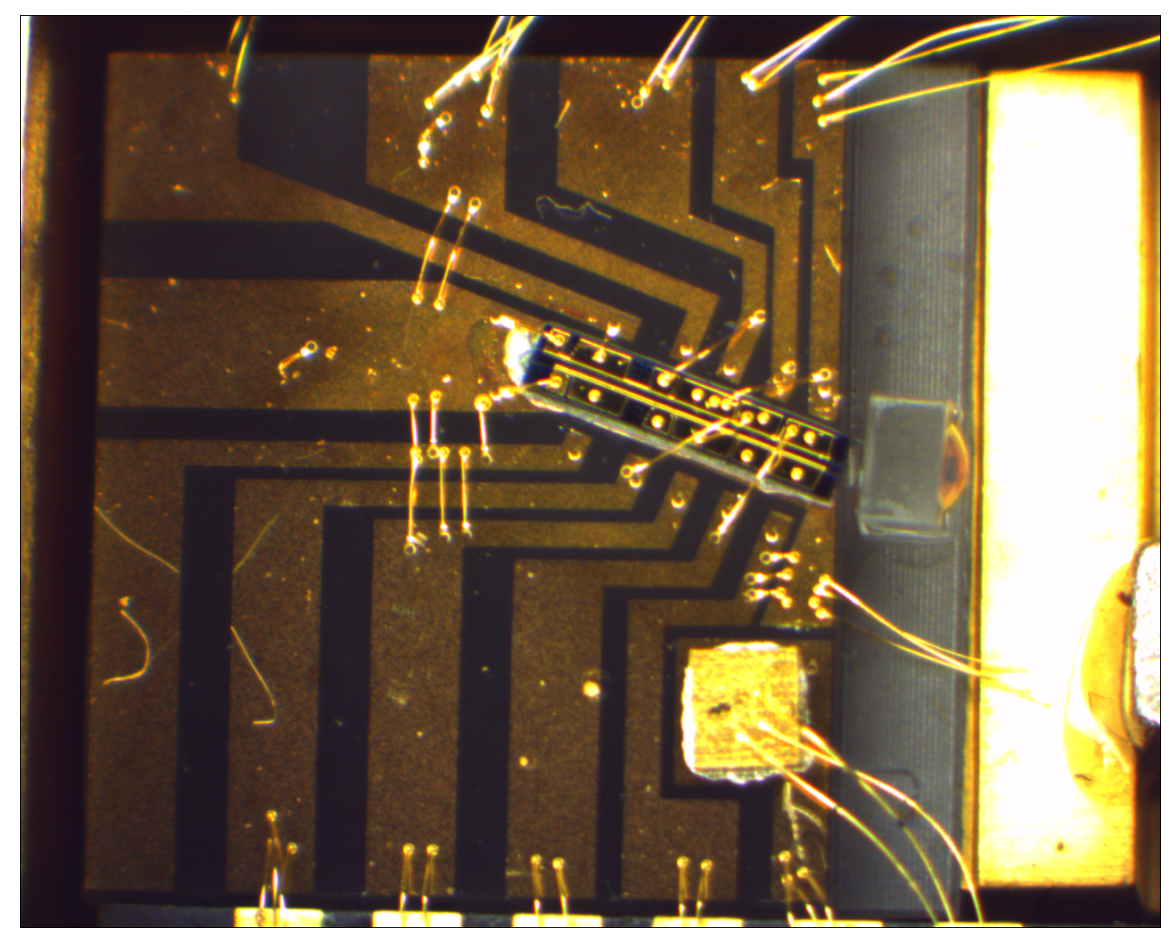

Figure 4.1: Casing removed from top of the SG-DBR laser to reveal the semiconductor structure and wire bonding. Notice the laser structure is angled with respect to the primary lens to reduce reflections. The thermistor for temperature feedback is indicated by the small square lower right below the laser. The thermal electric cooler (not visible) is directly beneath the laser structure. Approximate size of just the laser structure is $0.5 \times 2.5 \mathrm{~mm}$. 
A matched $50 \Omega$ environment is created through the use of microstrip transmission lines on the PCB to edge mount SMA connectors. A typical microstrip line has the signal trace routed on the top layer, with a solid reference plane directly below it, in this case the ground plane, separated by the particular dielectric. In order to create the $50 \Omega$ traces, the geometry of the microstrip lines is calculated according the relative permittivity $\epsilon_{r}$ of the substrate, height of the substrate $H$, thickness of the copper traces $t$, and width of the trace $W$ as in equation 4.1. The only variable under control for the PCB design process, given fixed parameters from the board chosen, is trace width $W$. The standard spec two layer PCB from Advanced Circuits was chosen, with $H=62$ mil, $t=1.4$ mil for a standard $1 \mathrm{oz}$ copper coverage [15], and $\epsilon_{r}=4.25$ [16]. End launch SMA connectors from Emerson Controls were chosen as a standard and compact RF connection to the Vector Network Analyzer (VNA) as well other electrical test equipment. According to [17], given identical specifications of board thickness, copper pour, and $\epsilon_{r}$ the trace width is specified by the manufacturer as 103 mil to achieve minimal VSWR and wide bandwidth. Calculated values yield $Z_{0}$ of approximately $55 \Omega$, within specified values from the manufacturer.

$$
Z_{0}=\frac{120 \pi}{\sqrt{\varepsilon_{e f f}} \times\left[\frac{W}{H}+1.393+\frac{2}{3} \ln \left(\frac{W}{H}+1.444\right)\right]}
$$

A 3D rendering of the PCB is shown in 4.2, partially populated with components from the DipTrace PCB library. For Gerber files used in layout, refer to appendix C. The laser mounts in the center of the board, with the portion labeled remove and surrounding areas manually routed away via a coping saw and smoothed with files. The fiber optic pigtail connector exits above the surface of the PCB, allowing for the delicate bare fiber to be taped down for support in the blank space above. The flat base of the laser rests below the PCB and attaches 
to an aluminum heatsink. It is essential the laser maintains a stable temperature. The oversized metal base acts as a stabilizing thermal mass for suppressing temperature transients. Both edges of the metal support bridge on the package fan out and rest on the large metal pads. Spacers are inserted in each of the four PCB mounting holes to raise the PCB surface above the heatsink and allow the SMA connectors to not interfere with the mounting plate below. When mounted onto the aluminum heatsink, the bolts provide slight mechanical pressure downward, making contact of the grounded chassis bridges onto the parallel traces on the board as shown in figure 4.3. The large parallel traces are periodically viaed down to the microstrip ground plane, helping to reduce inductance to ground.

When assembling the laser onto the testing fixture, and handling the laser in general, extreme caution must be taken in safeguarding against electrostatic discharge (ESD) to the delicate diodes. Modern diodes are designed to respond rapidly to changing voltage and current; a great performance aspect but also a liability for their operating conditions. ESD is typically created when a person is walking, especially on a carpeted floor, and then touches an object. Often people associate this with wearing a wool sweater and vigorously rubbing socks on carpet, but even normal clothing can unknowingly create a discharge in the range of 4,000-32,000 V DC, well over the operating voltage of 2-3 V [18]! Therefore, during assembly the Weller WD-1000 ESD safe soldering iron, combined with ESD wrist guards, and ESD protection work surfaces were used to mitigate the possibility of ESD transferring to the package. Whenever handling the testing fixture for transportation in the lab, shorted end cap SMA connector are affixed to prevent accidental discharge along with storage in an ESD safe bag. As an additional safeguard, for all measurements an ESD wristguard is worn and connected to the front panel ground port of the VNA to eliminate discharges. 




Figure 4.2: 3D Rendering of PCB design for laser breakout



Figure 4.3: Assembled laser breakout PCB, with TEC control populated, and mounted on 0.25 " aluminum heatsink. 


\subsection{TEC Controller}

As with all semiconductor based laser systems, temperature stability greatly affects wavelength output. In operation the laser package is required to maintain a constant stable temperature to lower required input current and reduce optical drift. Integrated within the laser package is a NTC type thermistor, which varies resistance in response to temperature. This provides an accurate in situ temperature measurement of the laser die. To actively heat or cool the laser, a Thermal Electric Cooler (TEC) is integrated. This device operates as a Peltier Junction, either heating or cooling depending on the direction of current flow. TECs operate on the Peltier effect, when a current flows through the device it causes heat to be brought from one side to the other side. This transfer of thermal energy causes one side to cool down and the other to heat up, of which the heating side is connected to a thermal heatsink. The heatsink will keep that surface at the ambient temperature, while the other side continues to cool below ambient. While the efficiencies are not the highest for such devices $(\sim 15 \%)$ their primary advantages are small form factor and bidirectional temperature control capabilities compared to traditional refrigeration based systems [19].

Traditionally, a laser controller module with an integrated TEC controller is utilized to perform the temperature monitoring and regulation. A device such as the ILX Lightwave LDC-3470 provides excellent stability and control, however it is very cumbersome, expensive, and not suited for product integration. Design of an on board TEC controller for regulating the die temperature was integrated to minimize the overall required external required components for testing multiple laser modules.

The particular controller chosen was the Maxim MAX1968 power driver for 
Peltier TEC modules. The MAX1968 utilizes two synchronous buck regulators to provide bipolar $\pm 3 \mathrm{~A}$ outputs [20]. Cooling operations are defined when the device is sinking current into the TEC, and heating occurs when sourcing current. External part count is minimized due to the internal switching FETs, requiring only output inductors, diodes, an assortment of filter capacitors, and set point resistors. The entire module operates off of a single rail power supply, ideal for a packaged installation.

The MAX1968 is only a TEC controller, directly controlling the output current for the module given an external analog voltage from the Current Control Input, CTLI, pin supplied from an external source. For this design, a fully analog Proportional-Integral-Derivative (PID) is implemented in hardware to generate the CTLI signal for the controller.

The PID control loop takes in a thermistor voltage, biased from the $V_{r e f}$, and compares it to the setpoint, generating an error signal through a difference amplifier according to equation 4.2. The error signal is then fed into the PID control loop forming by the op amp U2 in figure 4.4. The desired temperature setpoint is chosen by adjusting the $10 \mathrm{~K}$ trimpot; shown by modifying equation 4.2 by letting error equal 0 , and rearranging into equation 4.3. For example, a desired temperature set point lock of $20^{\circ} \mathrm{C}$, typical for the SG-DBR laser used in telecom applications, equation 4.3 would require the setpoint voltage of 0.825 V. Component values for the PID loop were chosen based on the reference design [20] for a typical TEC module's poles and zeros. Refer to appendix B for full schematics for the PID, controller, and laser.

$$
\text { error }=1.5 \mathrm{~V}\left(\frac{R_{T} \times\left(R_{4}+R_{5}\right)}{R_{4} \times\left(10 k \Omega+R_{T}\right)}\right)-V_{\text {set }}\left(\frac{R_{5}}{R_{4}}\right)
$$




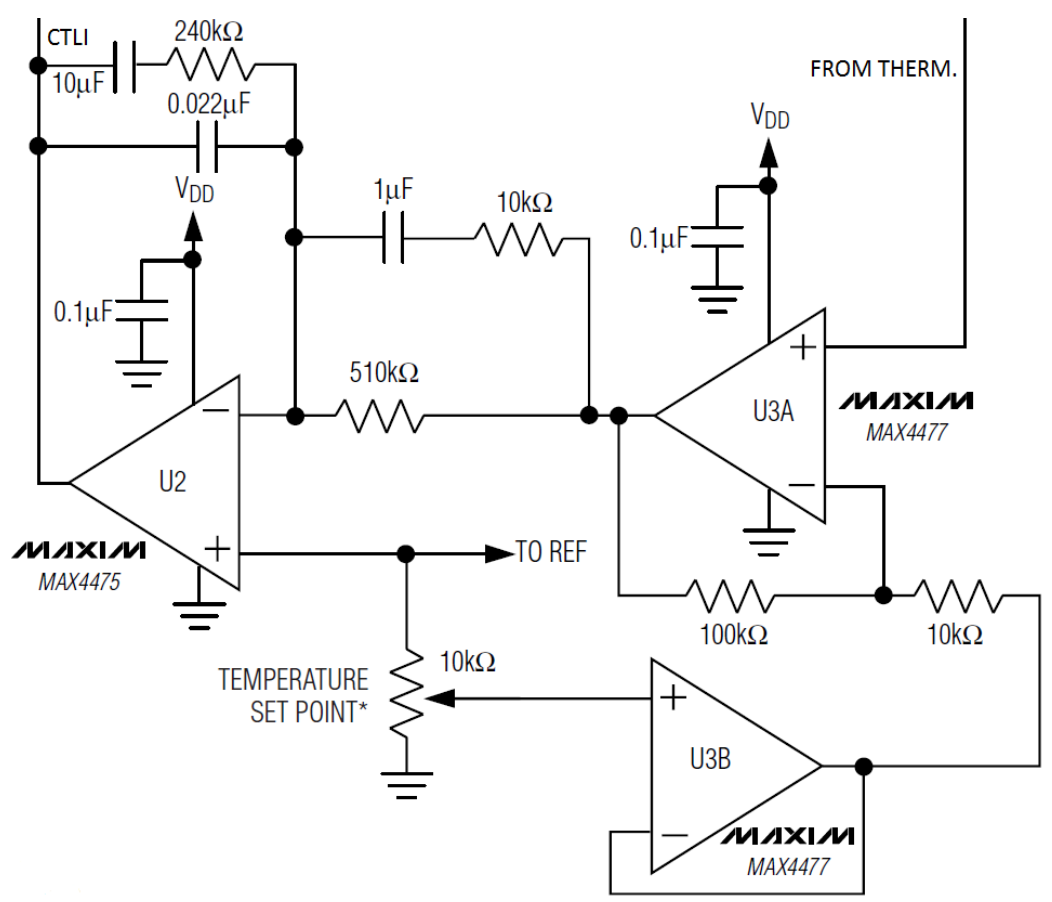

Figure 4.4: TEC PID Control Loop. The thermistor is biased with $V_{r e f}=1.50 \mathrm{~V}$ and a $10 \mathrm{~K} \Omega$ resistor. This input from the thermistor is compared to setpoint fed through U3B buffer from the $10 \mathrm{~K} \Omega$ trimpot on U3A. The main PID loop consists of U2, taking in the reference voltage on the noninverting terminal and comparing to the error signal generated from U3A. The CTLI output is sent to the TEC controller. When $V_{C T L I}=1.50 \mathrm{~V}$ there is no current flow, $V_{C T L I}<1.50 \mathrm{~V}$ device is heating, and $V_{C T L I}>1.50 \mathrm{~V}$ the device is cooling. For $20^{\circ} \mathrm{C}$ lock, the setpoint $V_{\text {set }}=0.825 \mathrm{~V}$. 


$$
V_{\text {set }}=\left(V_{\text {ref }}\right)\left(\frac{R_{5}}{R_{4}}\right)\left(\frac{R_{T} \times\left(R_{4}+R_{5}\right)}{R_{4} \times\left(10 k \Omega+R_{T}\right)}\right)
$$

Testing of the TEC followed a 2.5 week burn in of the board with a secondary laser module. Wavelength drift of less than $50 \mathrm{pm}$ occurred during the power cycle, indicating a drift of $\sim 0.5^{\circ} \mathrm{C}$ assuming a typical $0.1 \mathrm{~nm} /{ }^{\circ} \mathrm{C}$ temperature coefficient for similar SG-DBR devices [12]. Further optimization of the PID loop is necessary to obtain tighter temperature regulation and control, theoretically within $0.01^{\circ} \mathrm{C}$ as stated by Maxim with proper tuning [20].

Applying a step input to the temperature setpoint by adjusting the trimpot achieves a lock within less than 2 seconds, as observed by the voltage of the CTLI pin reaching $1.50 \mathrm{~V}$ with minimal oscillations. Proper current and voltage limits as configured by the external resistors agree with the design. However for the sake of characterizing the laser, the following tests of optical and electrical performance utilize the reference Lightwave source. The Maxim 1968 TEC controller chip performs as stated, regulating the temperature of the laser as designed but requires additional tuning and modifications to the control loop before it performs optimally. 


\section{Chapter 5}

\section{Equivalent Circuit Models}

The semiconductor laser of this study contains a diode for each of the front, back, phase, gain, and SOA sections connected in a common cathode configuration as show in appendix A. An understanding of each section's electrical performance is essential for quantifying the limits of operation for modulation of diode injection current. Once the $\mathrm{RC}$ parasitics are experimentally determined for each port, an evaluation of the operating frequency for modulation can be established.

\section{$5.1 \quad$ Test Setup}

It is often desired to have an evaluation of the reflection and transmission properties of high frequency components, such as the diode segments in the SGDBR laser, as a function of frequency. Ideally the widest band of frequencies is used for testing to obtain a complete characterization. There are two methods that can be used for characterization: a wide band frequency sweep or applying a fast rise-time voltage pulse into the device. Looking at the second method, 
applying a step input, and taking the Fourier Transform clearly shows the step contains a very broad range of harmonics [21]. In the ideal case this step function's frequency would extend from zero to infinity. However in practical applications, a finite frequency band exists. Therefore the shorter the rise-time, the broader the frequency band available to effectively sweep.

To investigate the the behavior of each diode section a technique known as Time Domain Reflectometry (TDR) is employed. A standard TDR device consists of a fast step generator feeding into a transmission line into the device under test (DUT). In between lies a sampling scope to analyse the reflections from the DUT. Instead of operating in frequency domain, TDR allows for analyzing reflections caused by discontinuities with respect to time or distance. Interconnects, packaging or breaks in cables can easily be resolved by their reflections separated in time. In fact, coaxial cables can be attached to concrete support structures for buildings to monitor for foundation stresses or fractures. Stresses in turn cause capacitive impedance changes on the cable, and able to be resolved in distance along the cable [22]. TDR is a relatively simple approach utilizes standard test equipment, however higher resolution TDR requires a specialized pulse generator and high speed oscilloscope.

A Vector Network Analyzer (VNA) is a specialized device to measure networks parameters of a device, such as reflection and transmission, commonly known as s-parameters. Typically the VNA is configured with a swept sinusoid stimulus to inject into the circuit, and then the reflections and transmissions are observed. While this method yields and accurate characterization of the complex impedance with respect for each frequency, an overall passive RLC model proves more difficult to obtain. A more intuitive approach is to use TDR to investigate each port's impedance. For this particular application the VNA is configured 
to operate in TDR mode instead of the swept sinusoid operation. In this VNA TDR based approach the true step response is calculated mathematically from a Fourier Series of swept harmonics. The end result from the VNA still however repents it as a classical TDR plot with time (or distance) on the abscissa, and reflection amplitude on the ordinate.

During testing all five sections of the laser are interchanged to the VNA port. A DC power supply is attached to the bias tee of the VNA to provide a DC bias during testing. The Anritsu MS4622 VNA is calibrated for reflection only measurements, known as $S_{11}$, in time harmonic series as shown in figure 5.1 with a photograph of the actual setup in figure 5.2. Equivalent circuit models are presented for each section for zero bias to maximum rated bias conditions. A MATLAB routine is used to plot the exported tabular data from the VNA and present all biasing currents overlaid.

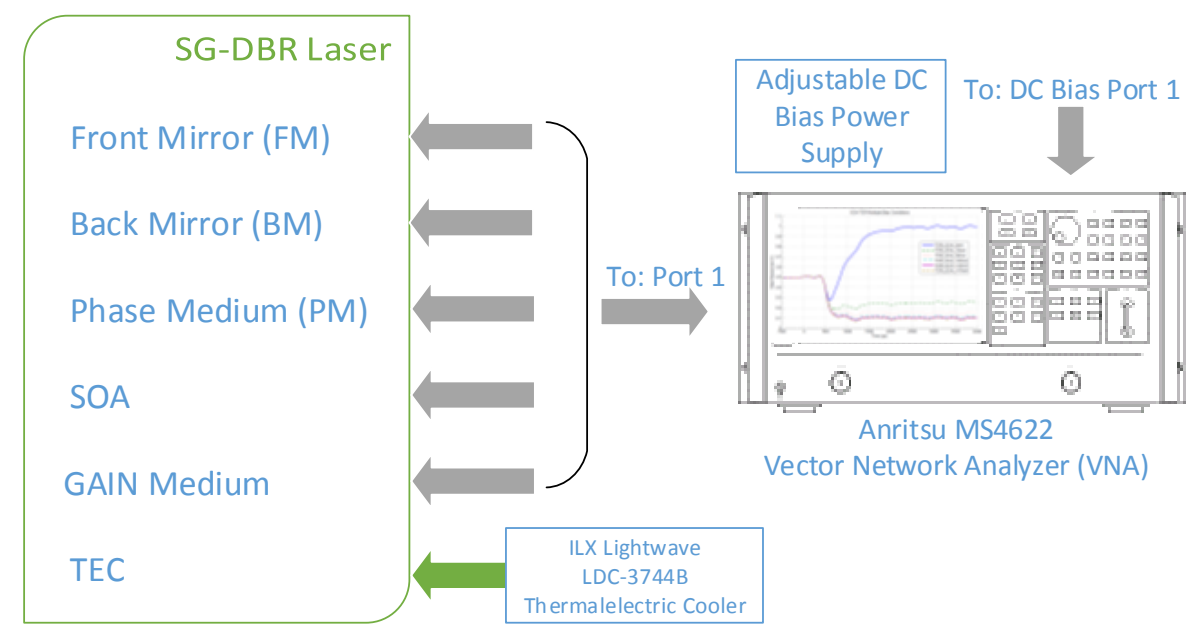

Figure 5.1: Circuit Model TDR Test Setup. Anritsu VNA configured in TDR mode, for harmonic series in range of $10 \mathrm{M}-3 \mathrm{GHz}$. Gray arrows indicate SMA connections, while green indicates standard shielded wire. DC bias source provided into port 1 external bias of the VNA. The ILX Lightwave TEC controller is configured for $20{ }^{\circ} \mathrm{C}$ lock. 


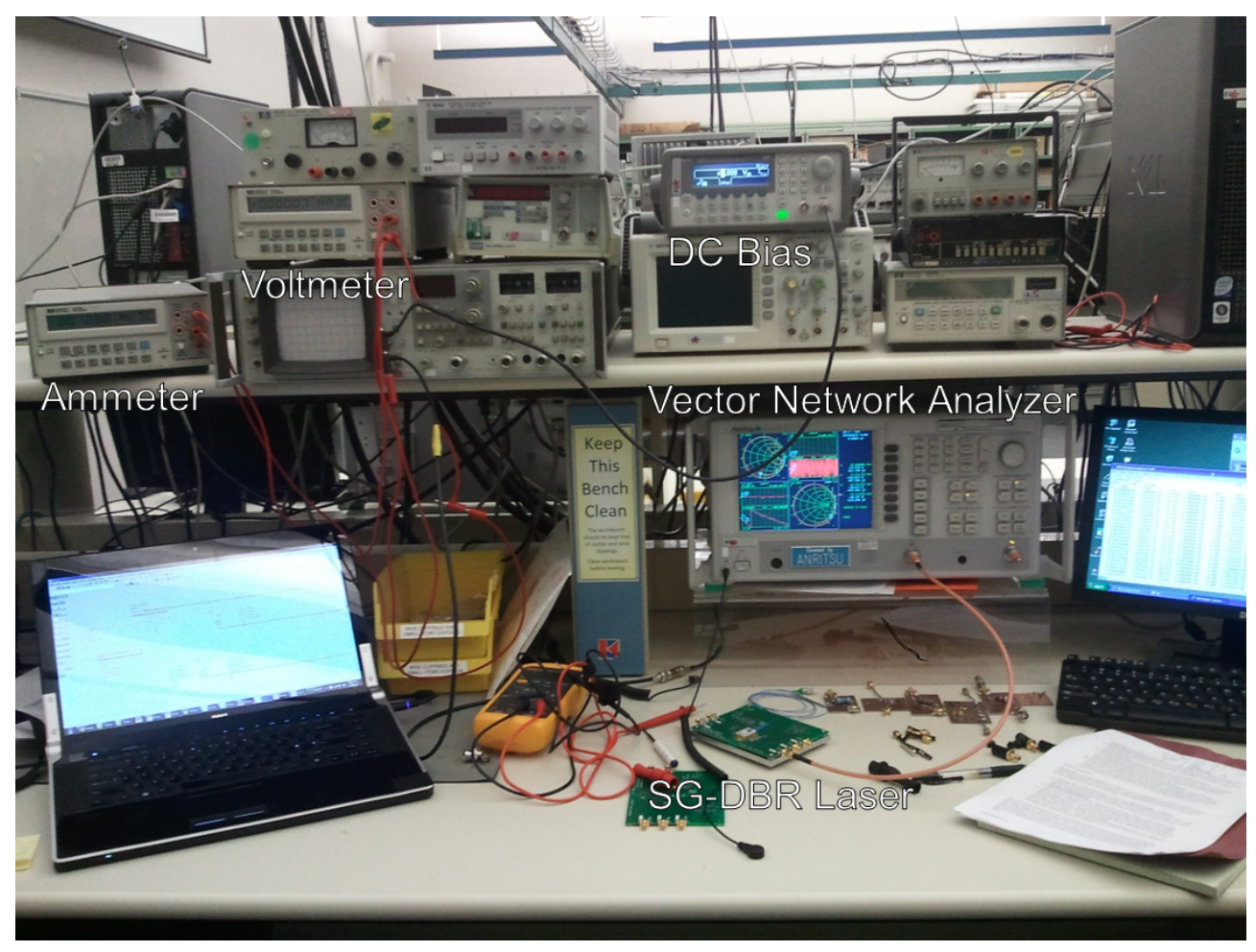

Figure 5.2: TDR Experimental Test Setup. VNA configured to operate in TDR mode, with external DC bias supplied from Agilent function generator into bias port 1 .

Modeling an ideal diode for forward bias is simply a short, and an open circuit for reverse bias. While this simplistic analysis is suitable for basic DC cases in a circuit, time varying signals require a technique known as small signal modeling must be performed. Typically the large signal analysis is too complex to be performed by hand, and requires an extensive model in a circuit simulation program. Small signal modeling of nonlinear devices facilitates their analysis by hand. Essentially the analysis is split into DC and AC signals, and then combined using superposition. A purely resistive model of the diode is valid for DC cases only, represented by $r_{d}$. This is known as the dynamic resistance, which is represented by the inverse slope of the tangent line at a specific bias on the IV curve [23]. The value changes depending upon the particular DC bias used, 
hence the name dynamic.

For high frequency modeling of the diode the simple resistive DC case will only function properly when the frequency is very low. When operating at higher frequencies the electric field inside is also time varying, causing a displacement current. The end effect of displacement currents are modeled by inserting equivalent capacitance into the existing model, as shown in figure 5.3. Two primary sources of capacitance are accounted for in the model. The depletion, or junction capacitance, is associated with the charge variations in the depletion layer and is denoted as $C_{j}$. The diffusion capacitance is associated with the diffusion of charge carriers in the device, denoted $C_{d}$. Notice both $C_{j}$ and $C_{d}$ are presented in parallel with $r_{d}$. The total capacitance is represented by

$$
C_{\text {total }}=C_{d}+C_{j}
$$

as the sum of both individual capacitance values. When in reverse bias or small forward bias, the junction capacitance $C_{j}$ dominates over the diffusion capacitance. However when in strong forward bias $C_{d}$ will be the dominating source of capacitance [23]. Therefore in the following analysis for the cases of zero bias, it is assumed to be entirely composed of the junction capacitance. A $\star$ in the IV curves indicates datapoints taken in TDR plots. 


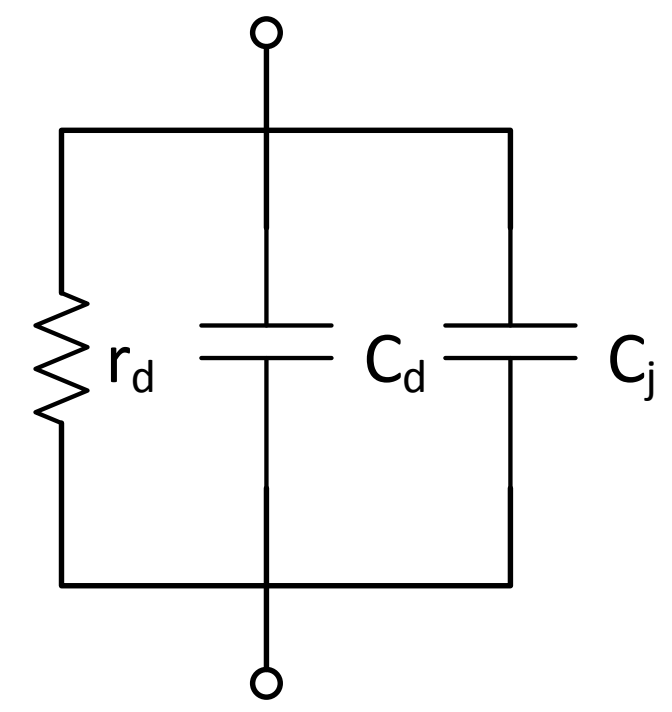

Figure 5.3: Small Signal Diode Model contains dynamic resistance $r_{d}$, junction (depletion) capacitance $C_{j}$, and the diffusion capacitance $C_{d}$.

\subsection{Front Mirror}

The current to voltage transfer characteristic of the front mirror is presented in figure 5.4. The figure is obtained from a simple ammeter and DC power supply, and manually recording the series of current vs. voltage points. The figure illustrates the diode current $I_{D}$ with respect to the diode voltage $V_{D}$.

The TDR responses for various bias currents are in figure 5.5, ranging from 0-35 mA. The zero bias case the diode appears like solely as a capacitor, with the resistance ideally infinite. Initially capacitors appear as short circuits. This brings the TDR graph down ideally to zero. However due to the finite risetime of the particular TDR used, the response does not reach entirely down to zero. The risetime is calculated to be 158 ps as measured at the $10-90 \%$ risetime of an open load. For calculation purposes, the valley of the dip is translated down to the ideal location of a short for time measurements. According to the behavior of a 




Figure 5.4: Front Mirror I-V curve.

capacitor, at $t=\infty$ a charge is fully present indicating an open circuit. Therefore the time constant $\tau$ is obtained from the initial charge position of 0 (short), to $63 \%$ of the maximum (open), and the time difference taken. The relation of time constant for a simple $\mathrm{RC}$ circuit is

$$
\tau=R \cdot C
$$

Where $\mathrm{R}$ is the load resistance, and $\mathrm{C}$ is the lump capacitance. Assuming a $50 \Omega$ load from the transmission line for $\mathrm{R}$, and rearranging 5.2 to solve for capacitance yields approximately $5 \mathrm{pF}$ for the zero bias case. For the forward biased cases herein, each behaves nearly identically as simple real impedance at steady state, indicating the particular dynamic resistance values for each bias. 
Therefore for simplicity of analysis, each will be treated as a resistor with the particular $C_{j}$ obtained earlier added in parallel. The approximate bandwidth of the $\mathrm{RC}$ circuit is

$$
f_{3 d B}=\frac{1}{2 \pi R C}
$$

where the values of $\mathrm{R}$ and $\mathrm{C}$ are $r_{d}$ and $C_{\text {total }}$, respectively, from the small signal model. For instance, in the case of $5 \mathrm{~mA}$ biasing current, the approximate model contains $r_{d}=32.3 \Omega$, and $C_{\text {total }}=5 \mathrm{pF}$ yielding $985 \mathrm{MHz}$ bandwidth. A full summary is presented in table 5.1.

Table 5.1: Front Mirror Circuit Summary

\begin{tabular}{|c||ccc|}
\hline$I_{\text {bias }}(\mathrm{mA})$ & $C_{\text {total }}(\mathrm{pF})$ & $r_{d}(\Omega)$ & Cutoff $(\mathrm{MHz})$ \\
\hline \hline 0 & 5 & 4900 & 6.50 \\
\hline 5 & 5 & 32.3 & 985 \\
\hline 10 & 5 & 19.0 & 1675 \\
\hline 20 & 5 & 14.5 & 2195 \\
\hline 30 & 5 & 11.8 & 2697 \\
\hline 35 & 5 & 10.9 & 2920 \\
\hline
\end{tabular}




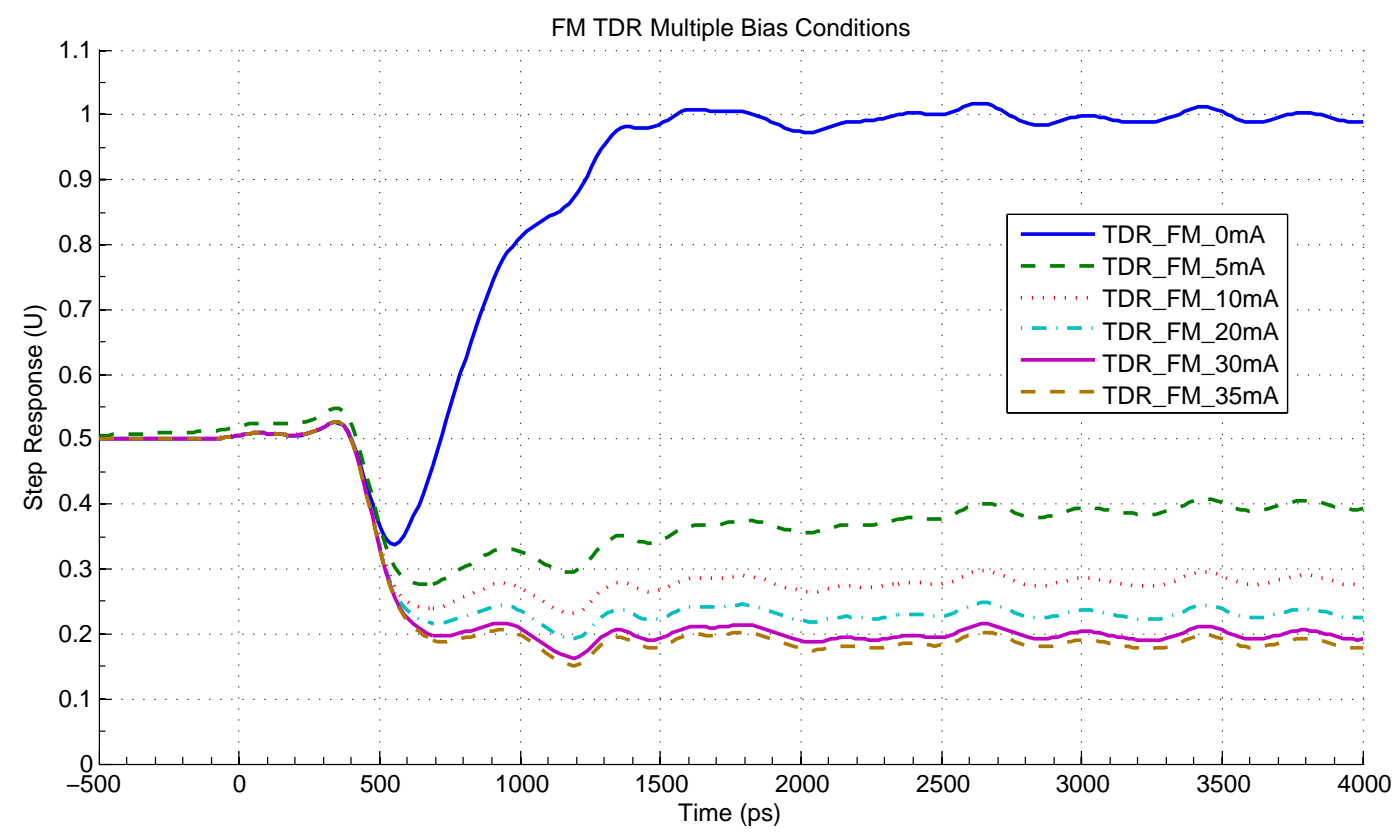

Figure 5.5: Front Mirror TDR Bias. The $0 \mathrm{~mA}$ bias case, presented in solid blue on top, illustrates the initial capacitive dip at approximately 550 ps. For analysis purposes this dip is translated down to the ideal initial short location of 0 . As the capacitor charges it behaves as an open circuit, settling at $1 . \mathrm{C} \approx 5 \mathrm{pF}$ for $0 \mathrm{~mA}$. Various bias current of $5,10,20,30$, and $35 \mathrm{~mA}$ are presented. Each behaves primarily as a resistor, decreasing in value with respect to bias current at steady state. Approximate values of $r_{d}$ are 32.2, 19, 14.5, 11.8, and $10.9 \Omega$ for above bias conditions, respectively. 


\subsection{Back Mirror}

As with the case of the front mirror, the back mirror's IV curve is presented in figure 5.6. The complete TDR response is in figure 5.7, with the summary table of equivalent circuit models presented in table 5.2.

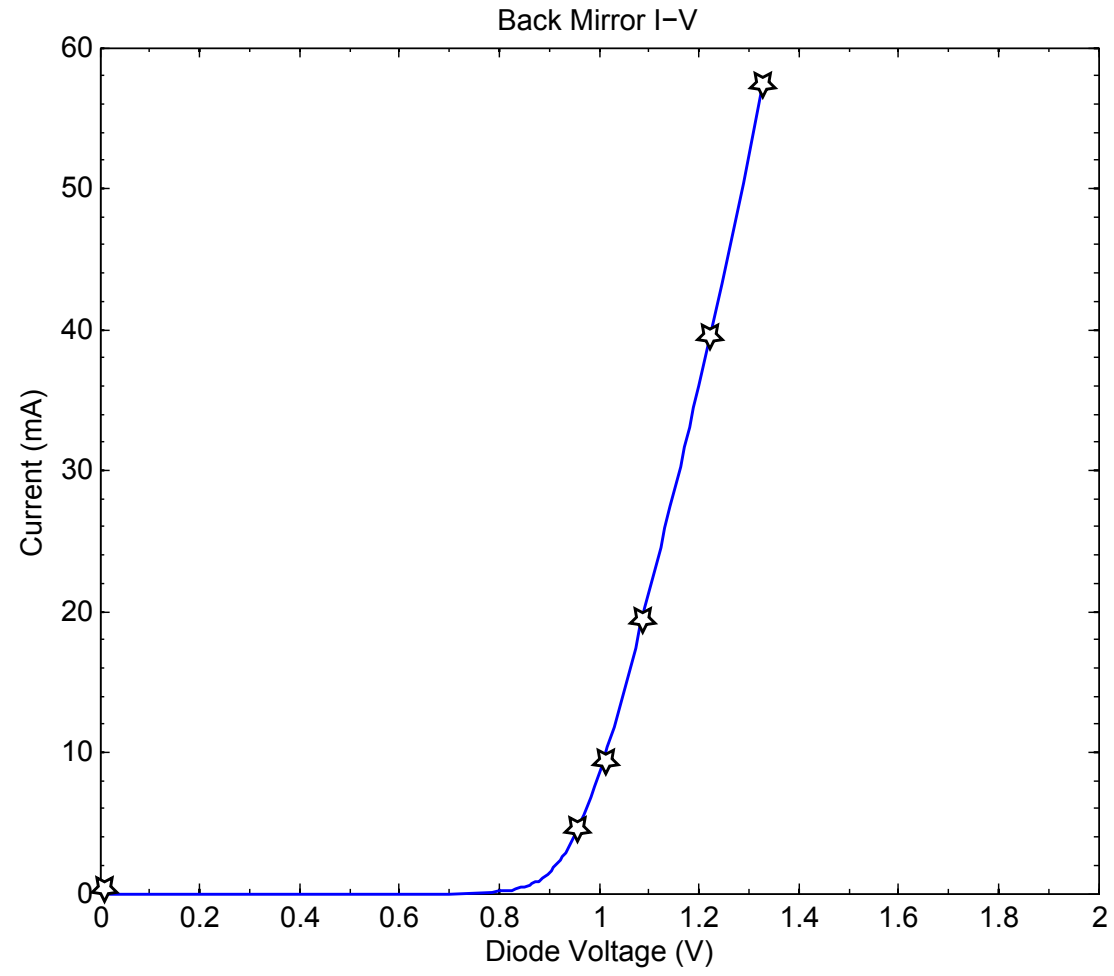

Figure 5.6: Back Mirror I-V curve.

Table 5.2: Back Mirror Circuit Summary

\begin{tabular}{|c||ccc|}
\hline$I_{\text {bias }}(\mathrm{mA})$ & $C_{\text {total }}(\mathrm{pF})$ & $r_{d}(\Omega)$ & Cutoff $(\mathrm{MHz})$ \\
\hline \hline 0 & 10.7 & 4117 & 3.6 \\
\hline 5 & 10.7 & 25.4 & 585.6 \\
\hline 10 & 10.7 & 9.5 & 1565 \\
\hline 20 & 10.7 & 7.3 & 2037 \\
\hline 40 & 10.7 & 6.4 & 2324 \\
\hline 60 & 10.7 & 5.9 & 2521 \\
\hline
\end{tabular}




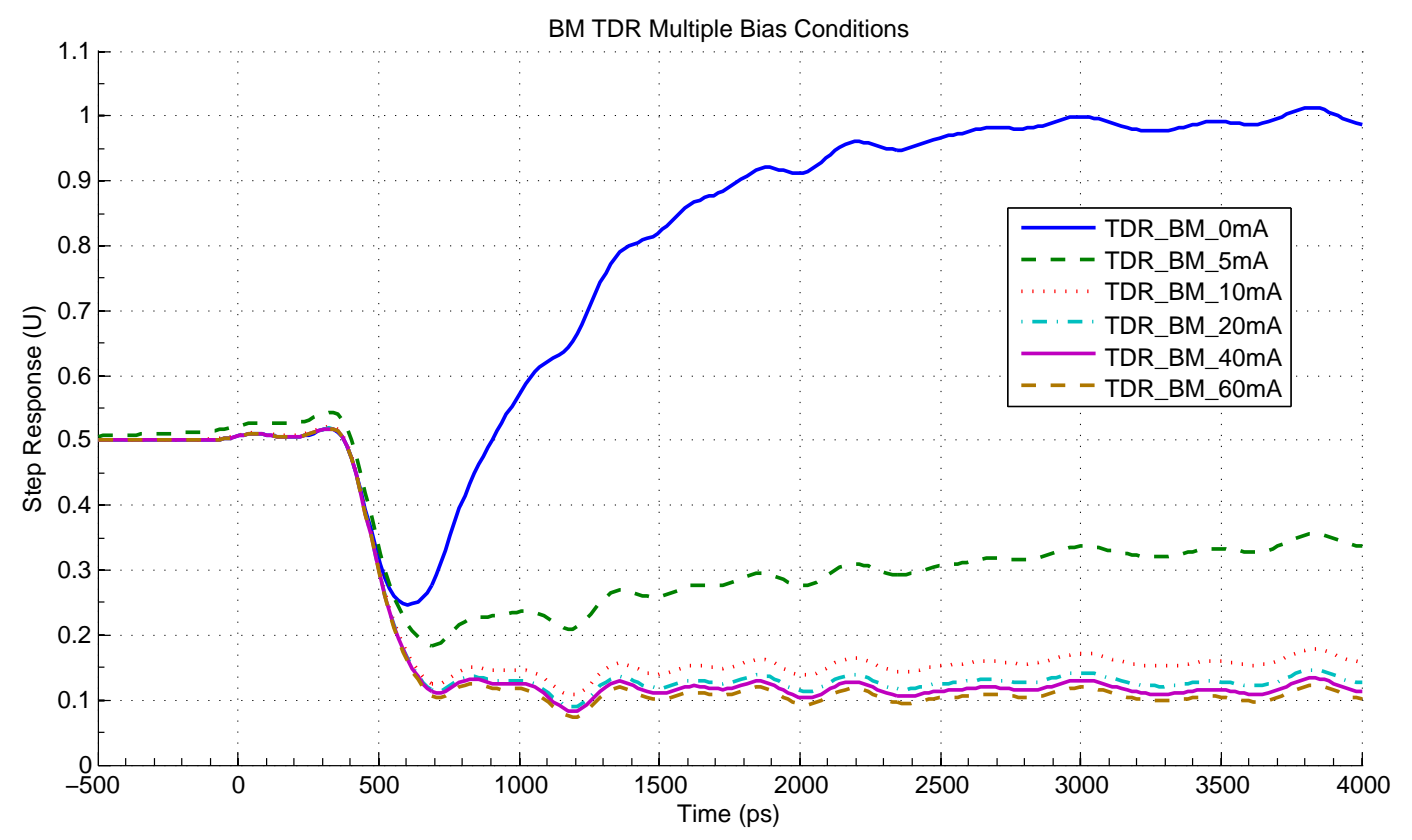

Figure 5.7: Back Mirror TDR Bias. The $0 \mathrm{~mA}$ bias case, presented in solid blue on top, illustrates the initial capacitive dip at approximately $600 \mathrm{ps}$. $\mathrm{C} \approx 10.7 \mathrm{pF}$ for $0 \mathrm{~mA}$. Various bias current of 5, 10, 20, 40, and $60 \mathrm{~mA}$ are presented. Each behaves primarily as a resistor, decreasing in value with respect to bias current at steady state. Approximate values of $r_{d}$ are $25.4,9.5,7.3,6.4$, and $5.9 \Omega$ for above bias conditions, respectively. 


\subsection{Phase}

The phase section's IV curve is presented in figure 5.8. The TDR response is in figure 5.9, with the summary table of equivalent circuit models presented in table 5.3. The capacitance scales appropriately with size of the diode segments. The phase being the smallest section with the lowest current rating also has the smallest effective capacitance of approximately $4 \mathrm{pF}$.

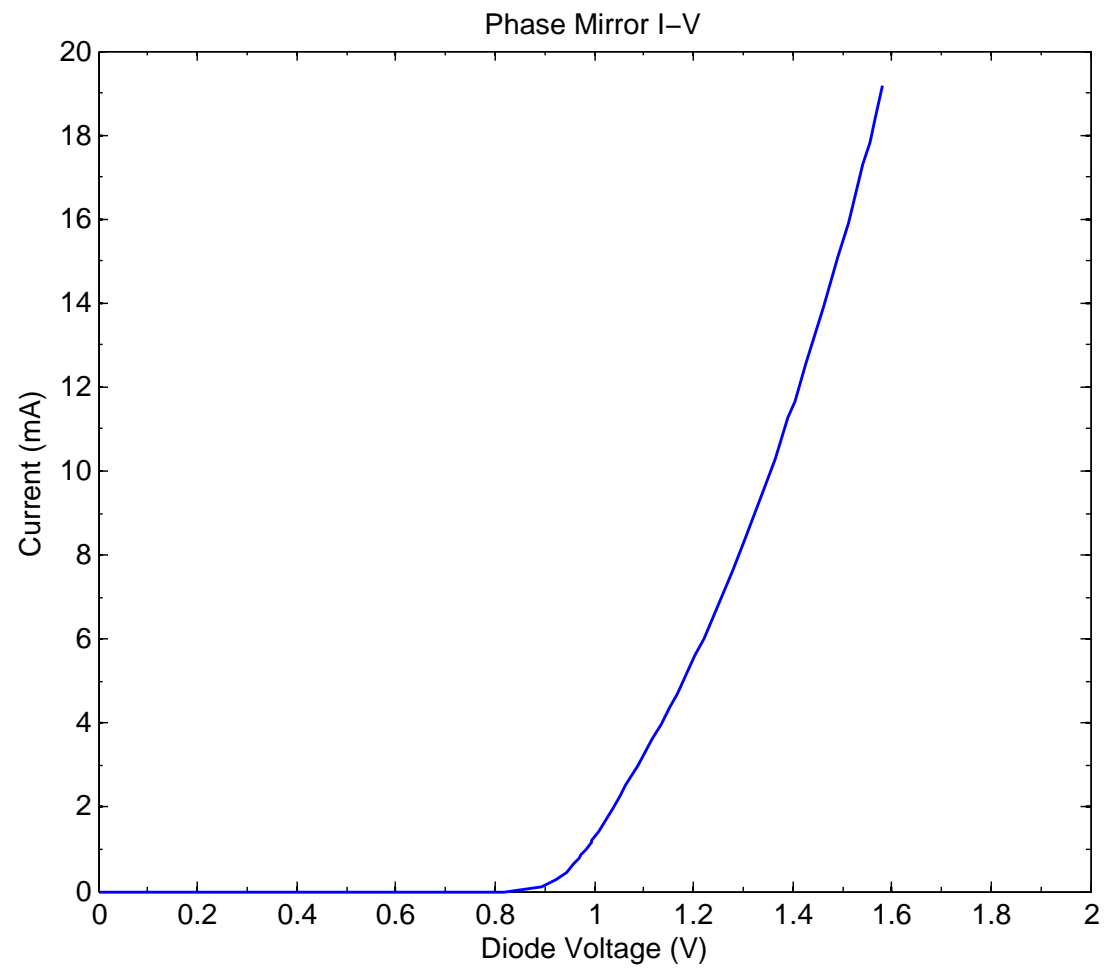

Figure 5.8: Phase Mirror I-V curve. 


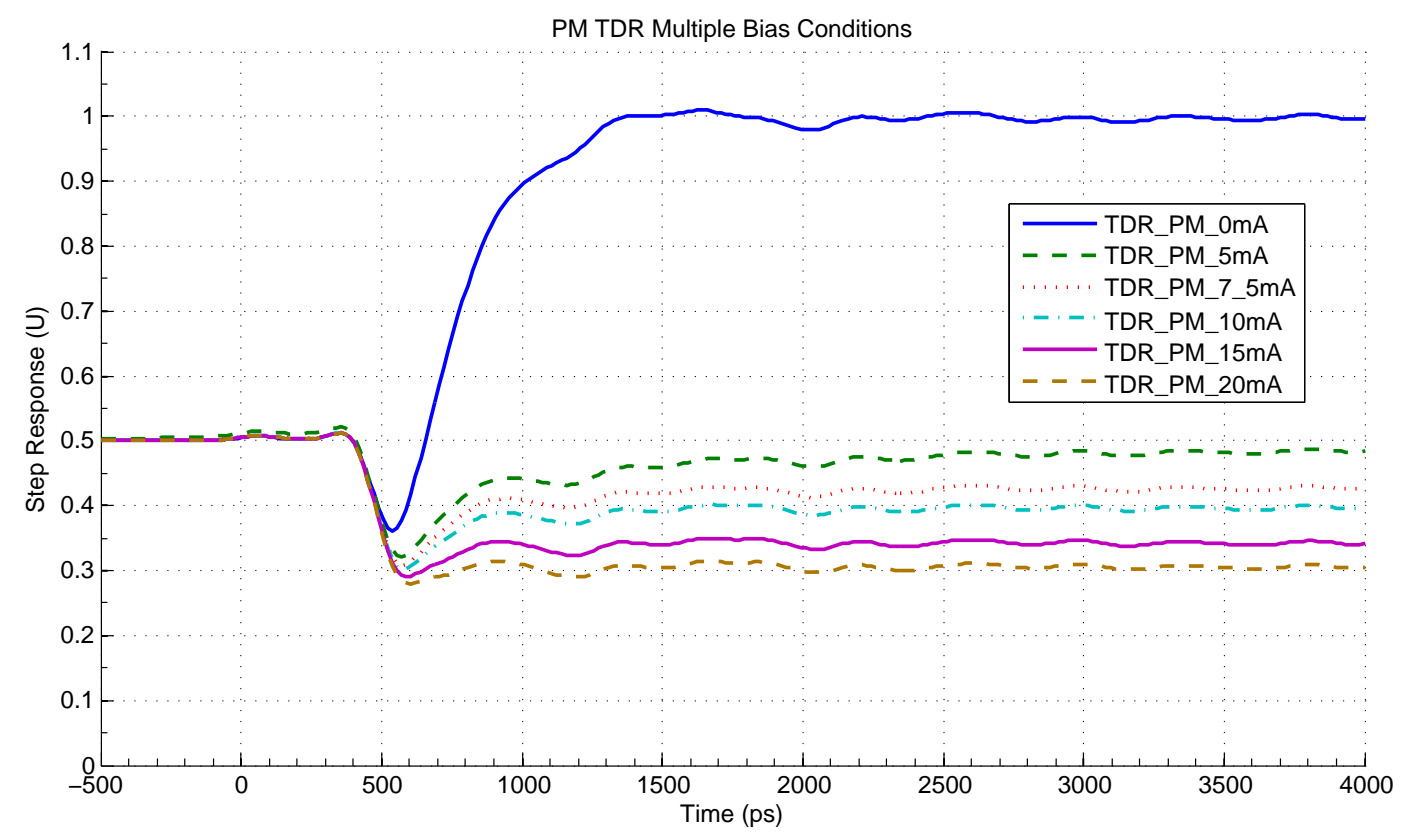

Figure 5.9: Phase Mirror TDR Bias. The $0 \mathrm{~mA}$ bias case, presented in solid blue on top, illustrates the initial capacitive dip at approximately $530 \mathrm{ps}$. $\mathrm{C} \approx 4 \mathrm{pF}$ for $0 \mathrm{~mA}$. Various bias current of $5,7.5,10,15$, and 20 $\mathrm{mA}$ are presented. Each behaves primarily as a resistor, decreasing in value with respect to bias current at steady state. Approximate values of $r_{d}$ are $46.9,37.1,32.8,25.9$, and $21.9 \Omega$ for above bias conditions, respectively.

Table 5.3: Phase Section Circuit Summary

\begin{tabular}{|c||ccc|}
\hline$I_{\text {bias }}(\mathrm{mA})$ & $C_{\text {total }}(\mathrm{pF})$ & $r_{d}(\Omega)$ & Cutoff $(\mathrm{MHz})$ \\
\hline \hline 0 & 4 & 16000 & 2.4 \\
\hline 5 & 4 & 46.9 & 848.4 \\
\hline 7.5 & 4 & 37.1 & 1072 \\
\hline 10 & 4 & 32.8 & 1213 \\
\hline 15 & 4 & 25.9 & 1536 \\
\hline 20 & 4 & 21.9 & 1816 \\
\hline
\end{tabular}




\subsection{Gain}

Again the TDR response for the Gain section is in figure 5.10. A summary table of equivalent circuit models presented in table 5.4. The Gain section exhibits much higher RC limited bandwidths compared to the mirror sections. At $125 \mathrm{~mA}$ the dynamic resistance is $5.5 \Omega$ with a capacitance of $9 \mathrm{pF}$ indicates a bandwidth of $3.2 \mathrm{GHz}$.

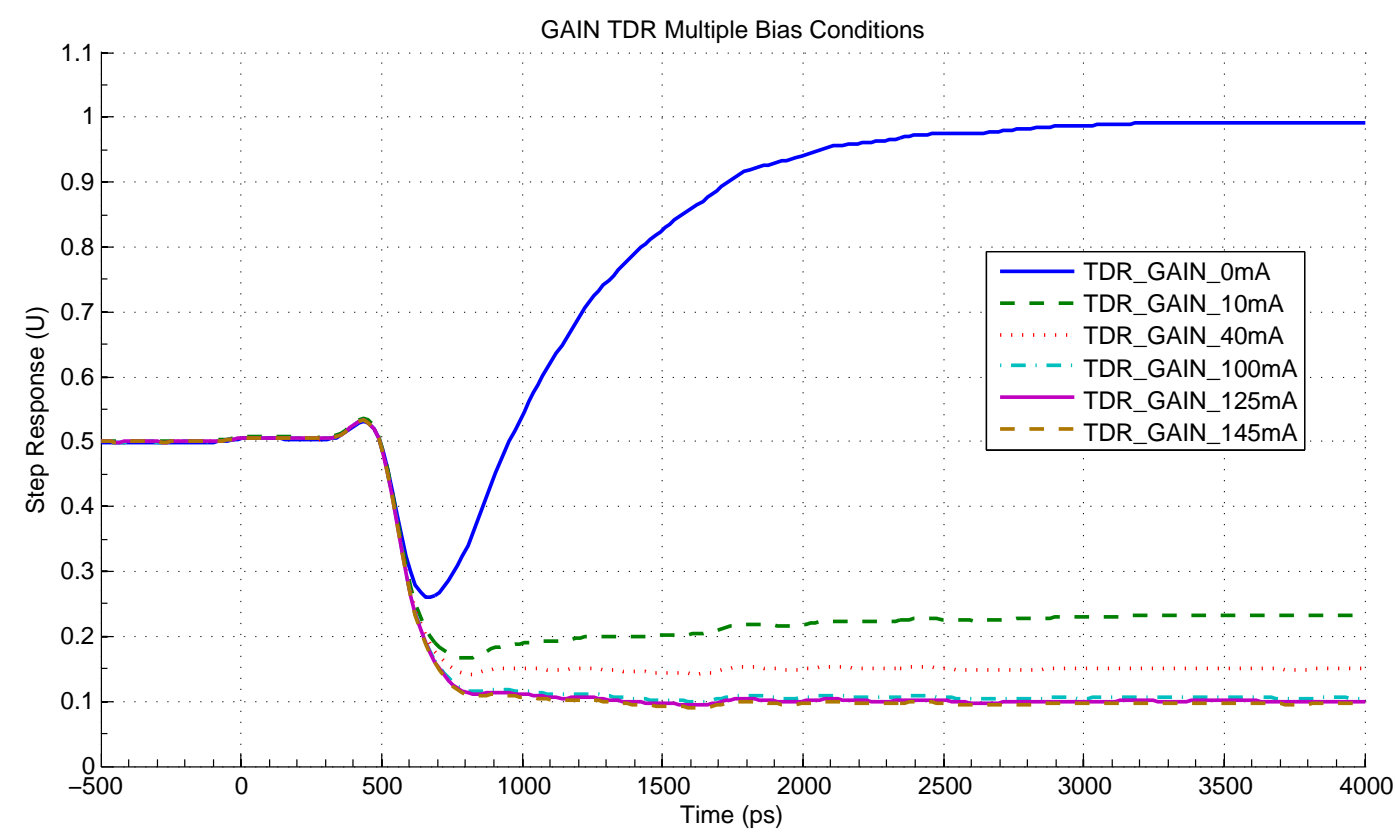

Figure 5.10: GAIN Section TDR Bias. The $0 \mathrm{~mA}$ bias case, presented in solid blue on top, illustrates the initial capacitive dip at approximately 670 ps. $\mathrm{C} \approx 9 \mathrm{pF}$ for $0 \mathrm{~mA}$. Various bias current of 10, 40, 100, 125, and $140 \mathrm{~mA}$ are presented. Each behaves primarily as a resistor, decreasing in value with respect to bias current at steady state. Approximate values of $r_{d}$ are 15.2, 8.8, 5.9, 5.5, and 5.3 $\Omega$ for above bias conditions, respectively. 
Table 5.4: Gain Section Circuit Summary

\begin{tabular}{|c||ccc|}
\hline$I_{\text {bias }}(\mathrm{mA})$ & $C_{\text {total }}(\mathrm{pF})$ & $r_{d}(\Omega)$ & Cutoff $(\mathrm{MHz})$ \\
\hline \hline 0 & 9 & 6200 & 2.9 \\
\hline 10 & 9 & 15.2 & 1163 \\
\hline 40 & 9 & 8.8 & 2009 \\
\hline 100 & 9 & 5.9 & 2997 \\
\hline 125 & 9 & 5.5 & 3215 \\
\hline 140 & 9 & 5.3 & 3336 \\
\hline
\end{tabular}




\subsection{Semiconductor Optical Amplifier}

The final SOA section's TDR response is in figure 5.11. A summary table of equivalent circuit models presented in table 5.5. Since the SOA is a higher current section, similar to the Gain the equivalent circuit model shows much smaller resistance values as current increases. For example the dynamic resistance is only $5.8 \Omega$ at $125 \mathrm{~mA}$ of current with the capacitance of approximately $7 \mathrm{pF}$ yields a RC limited bandwidth of $3.9 \mathrm{GHz}$.

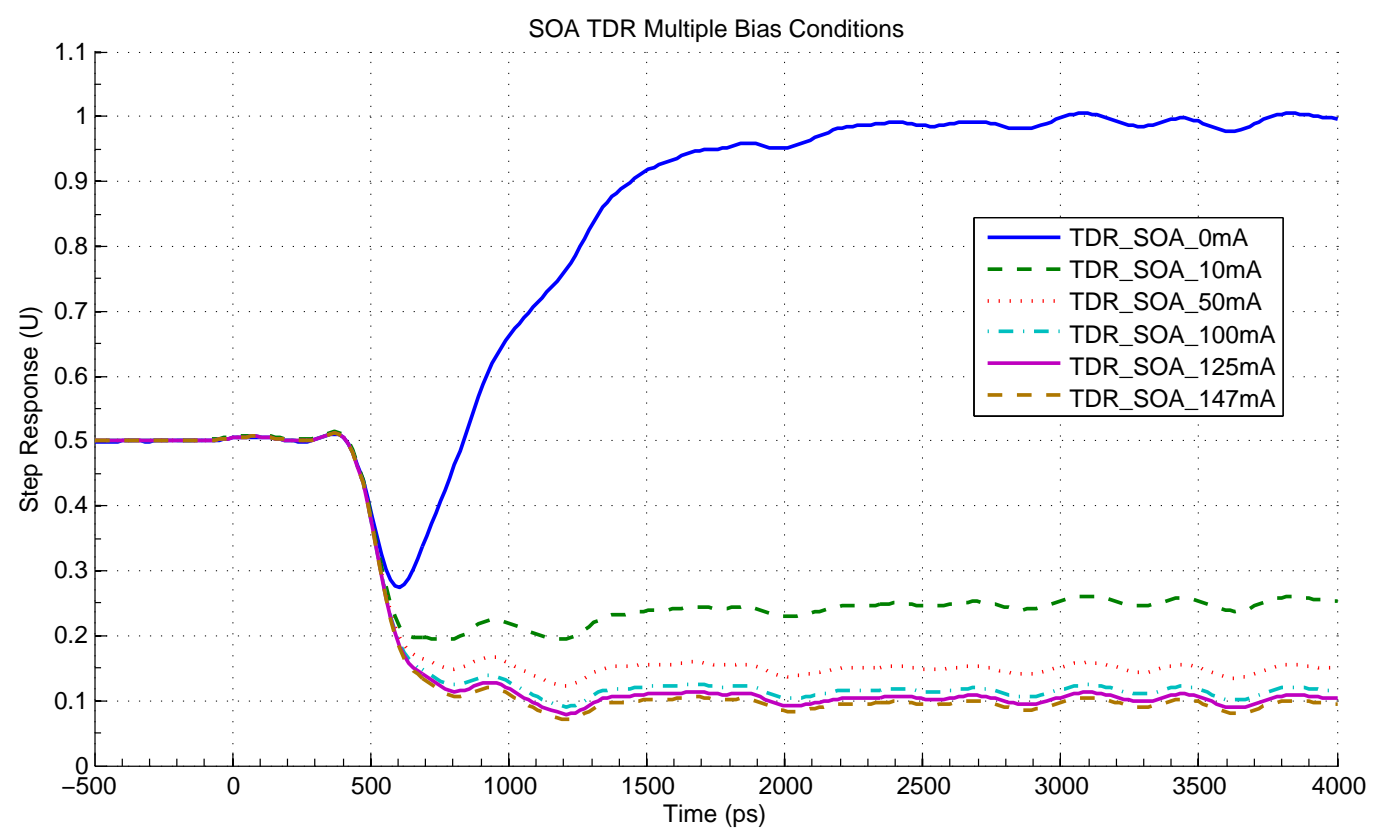

Figure 5.11: SOA Section TDR Bias. The $0 \mathrm{~mA}$ bias case, presented in solid blue on top, illustrates the initial capacitive dip at approximately $600 \mathrm{ps}$. $\mathrm{C} \approx 7 \mathrm{pF}$ for $0 \mathrm{~mA}$. Various bias current of 10, 50, 100, 125, and $147 \mathrm{~mA}$ are presented. Each behaves primarily as a resistor, decreasing in value with respect to bias current at steady state. Approximate values of $r_{d}$ are $17.1,8.8,6.5,5.8$, and $5.3 \Omega$ for above bias conditions, respectively. 
Table 5.5: SOA Section Circuit Summary

\begin{tabular}{|c||ccc|}
\hline$I_{\text {bias }}(\mathrm{mA})$ & $C_{\text {total }}(\mathrm{pF})$ & $r_{d}(\Omega)$ & Cutoff $(\mathrm{MHz})$ \\
\hline \hline 0 & 7 & 16000 & 1.4 \\
\hline 10 & 7 & 17.1 & 1329 \\
\hline 50 & 7 & 8.8 & 2583 \\
\hline 100 & 7 & 6.5 & 3497 \\
\hline 125 & 7 & 5.8 & 3920 \\
\hline 147 & 7 & 5.3 & 4289 \\
\hline
\end{tabular}

\subsection{Analysis}

In general, increasing bias current yields increased cutoff frequency according to the RC parasitic limited model. The gain and SOA sections contain smaller dynamic resistance values compared to the frequency dependent mirror sections, yielding higher predicted bandwidth. Capacitance also scales with the size and current capability of the diode segments. The larger current sections including the back, gain, and SOA sections similarly have some of the larger capacitance values. The phase section is the most delicate in terms of current, with a capacitance of $4 \mathrm{pF}$.

In nearly all of the TDR responses repetitive oscillations are present on all of the bias cases. This is likely attributed to a combination of reactive components creating an LC resonance. Since the same frequency is visible on each bias case this indicates a variable constant without dependence on bias current. Lead and package inductance are probable causes of the small oscillations on the output.

As an example, referring to figure 5.5, measuring several of the periods of oscillation for the front mirror on each bias current, and then averaging the results yields an oscillation frequency of approximately $2.60 \mathrm{GHz}$. Refer to table 5.6 for the summary of oscillation frequencies with respect to each section. Assume the additional inductance due to packaging, $L_{\text {package }}$, as series inductance to the 
existing parallel RC model. The resonant frequency of the RLC case

$$
\omega_{0}=\sqrt{\frac{1}{L C}-\frac{1}{(R C)^{2}}}
$$

Where $\omega_{0}$ is the natural frequency in radians, and $\mathrm{R}, \mathrm{C}$ are taken from the simplified equivalent circuit models. Rearranging 5.4 and solving for L yields

$$
L=\frac{R^{2} C}{\left(\omega_{0} R C\right)^{2}-1}
$$

Back to the front mirror example at $5 \mathrm{~mA}$, solving for $\mathrm{L}$ in 5.5 given $\omega_{0}=$ $2.60 \mathrm{GHz}$ produces an inductance of approximately $0.87 \mathrm{nH}$.

Table 5.6: TDR Oscillation Frequency Comparison

\begin{tabular}{|c||c|}
\hline Section & Oscillation Frequency $(\mathrm{GHz})$ \\
\hline \hline Front & 2.60 \\
\hline Back & 2.44 \\
\hline Phase & 2.53 \\
\hline Gain & 2.60 \\
\hline SOA & 3.23 \\
\hline
\end{tabular}

To verify this inductance source, the microscope picture was measured with a scale reference as shown in figure 5.12. The traces were mapped to the outside package pins to determine the correct sections. For the front mirror case, the length of the wirebond arching over the laser is approximately $1 \mathrm{~mm}$. For a typical wirebond diameter of $25 \mu \mathrm{m}$, the length of the wirebond $\ell$ in millimeters is approximately equal the inductance in nanohenries [24]. Therefore, for the 1 $\mathrm{mm}$ wirebond for the front mirror, $L \approx 1 \mathrm{nH}$. Using this value of inductance and solving for the natural frequency of equation 5.4 yields $f_{0} \approx 2 \mathrm{GHz}$. Table 5.7 summarizes the inductance $L_{\text {calcTDR }}$ from the oscillations on the TDR plots, $L_{\text {estimate }}$ from the physical length of wirebonds, and the oscillation measured from 
the TDR response as well as the calculated oscillation frequency using $L_{\text {estimate }}$.

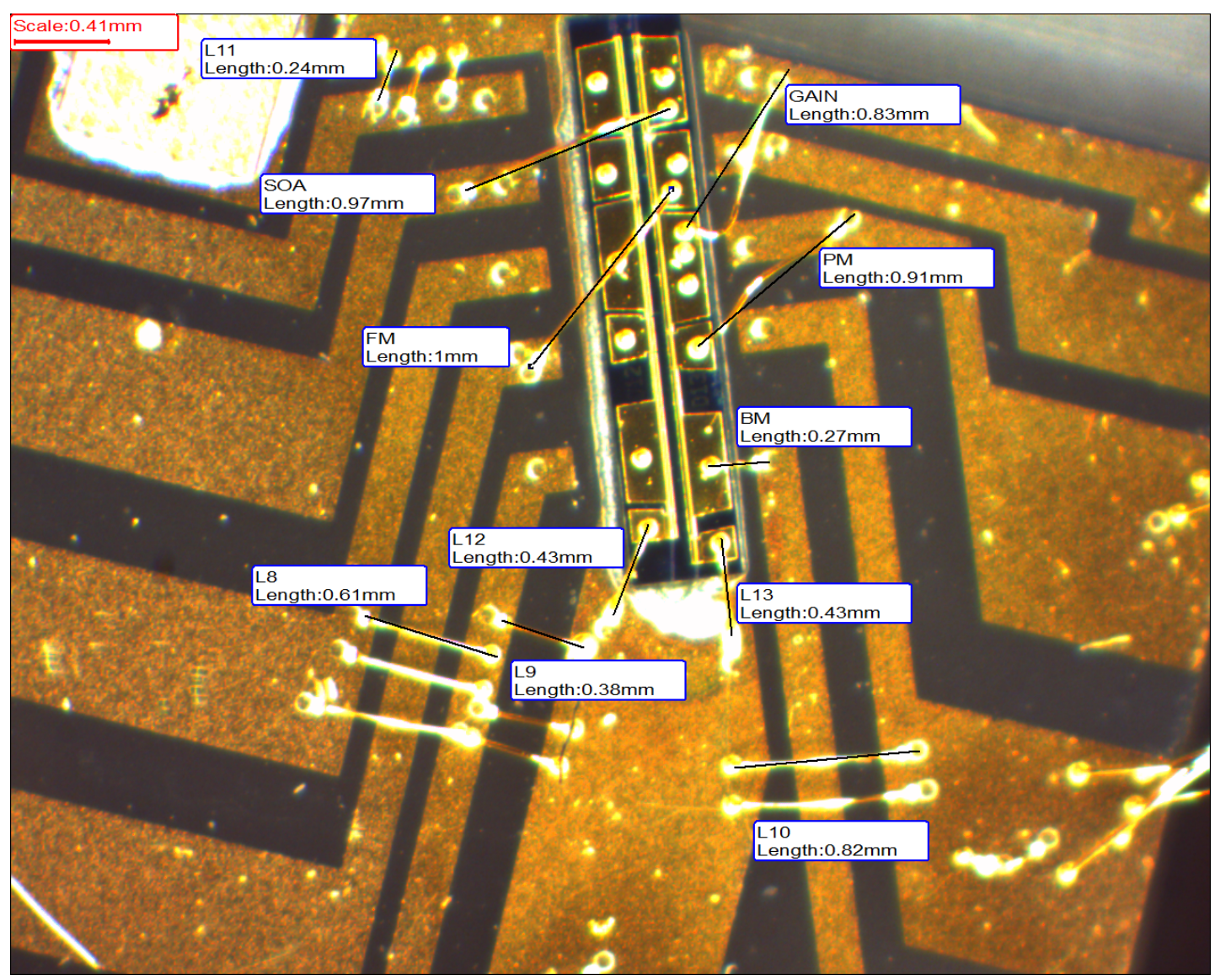

Figure 5.12: Wirebond Length Measurement. Approximate lengths of wirebonding to the laser sections. Red scale bar is $0.41 \mathrm{~mm}$.

The calculated and measured values of oscillation frequency are well within the same order of magnitude of each other, and agree reasonable well given the rough approximation for wirebond estimation. However a matching network likely exists between the laser die, the substrate, and the leads. The true total inductance is therefore larger, but is controlled via the matching network. A deeper understanding of the particular substrate used, and more accurate measurements of the wirebonds is necessary to obtain the full cause of the small oscillations present. 
Table 5.7: Inductance Induced Oscillation Comparison

\begin{tabular}{|c||c|c|c|c|}
\hline Section & $L_{\text {calcTDR }}(\mathrm{nH})$ & $L_{\text {estimate }}(\mathrm{nH})$ & TDR Meas. (GHz) & Calc. $(\mathrm{GHz})$ \\
\hline \hline Front & 0.87 & 1.00 & 2.60 & 2.02 \\
\hline Back & 0.42 & 0.27 & 2.44 & 2.30 \\
\hline Phase & 1.21 & 0.91 & 2.53 & 2.49 \\
\hline Gain & 0.61 & 0.83 & 2.60 & 1.45 \\
\hline SOA & 0.86 & 0.97 & 3.23 & 1.40 \\
\hline
\end{tabular}




\section{Chapter 6}

\section{Amplitude Modulation (AM) Bandwidth Characterization}

The wavelength is not the only element requiring tuning for sweeps. As the wavelength swept, the intensity fluctuates depending on the mode. The intensity must therefore be constantly adjusted to maintain a constant power level. Intensity, or Amplitude Modulation (AM) is one of the simplest methods for modulation of data, in this case optical output intensity from the laser. In the optical domain there are two primary approaches to modulation, which include direct modulation or external modulation. Direct modulation, as the name im-

plies, involves directly controlling the bias current to the laser thereby controlling the optical output power. The second method involves maintaining a constant optical output power of the laser and feeding the output into an external modulator, such as a Mach-Zehnder modulator, applying the intensity modulation at that point. Luckily in the case of DFB and SG-DBR lasers, direct modulation is possible due to the double heterostructure design. When a biased above threshold, output optical power will be proportional to bias current [25]. 
In the case of the SG-DBR, there are two amplitude dependent sections the gain and the SOA. Varying the bias current into each of these sections under modulation will result in shifting of the maximum modulation frequency, as will be demonstrated later on. Additionally, the conversion efficiency, also known and the slope responsivity $R_{S}$, relates the ratio of modulating current to the laser's output optical modulation. Essentially this describes how a change in input current yields a particular change in output optical power. Of course the responsivity is a function of modulation frequency. With an increase in frequency, the conversion efficiency will worsen. The standard metric of $-3 \mathrm{~dB}$ quantifies the laser's modulation bandwidth [25].

\subsection{Test Setup}

Figure 6.1 below provides a high level overview of the experimental testing setup. The laser is configured with fixed bias to the front, back, and phase sections provided by DC offset mode of the Agilent 33220A waveform generators with current limiting resistors and protection diodes in place on the output terminals. For biasing the gain section a simple linear power supply is used, in an attempt to reduce noise being transferred onto the gain port. The SOA section is also powered by the DC mode of the waveform generators for simplicity. In general, the SOA bias is fixed for every every experiment at $4.00 \mathrm{~V}$, or roughly $98 \mathrm{~mA}$, and rarely is adjusted besides the amplitude modulation tests herein. Depending upon the bias test (gain or SOA), the bias supply is switched to the external bias port of the VNA. A photograph of the testing arrangement is presented in figure 6.2 .

Out of the laser's single mode fiber optic ST connector, an isolator is inserted 
to reduce reflections from traveling back into the source. It works on the principle of a $45^{\circ}$ Faraday rotator. The input wave is first vertically polarized coming into the box, and then rotated $45^{\circ}$ to the output. Any waves traveling in from the output port will be rotated $45^{\circ}$ in the same direction, and therefore be $90^{\circ}$ total. This opposite traveling wave is horizontally polarized and attempts to pass through the initial vertical polarizer, and is eliminated.

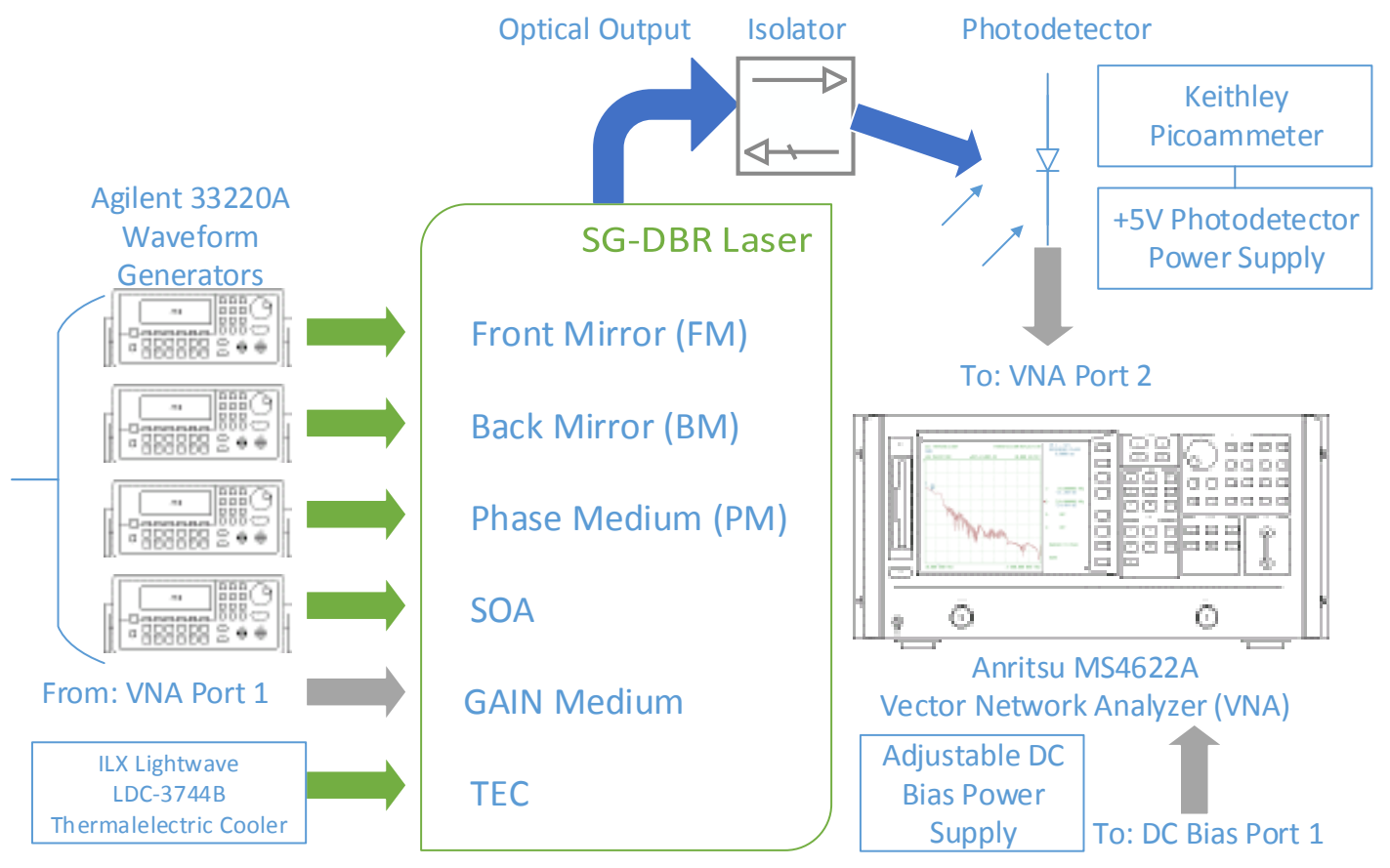

Figure 6.1: Amplitude Modulation (AM) Experimental Test Setup. DC electrical lines are denoted with green arrows, SMA based coaxial connections with gray arrows, and fiber optical links with blue arrows. The VNA modulates the gain or SOA ports with swept sinusoids. A $10+\mathrm{GHz}$ bandwidth photodetector receives the modulated light, converting it into electrical, back into port 2 of the VNA. $S_{21}$ plot is displayed on the VNA. External DC bias is supplied via rear port 1 of the VNA. All function generators are adjusted accordingly to achieve a stable mode. A Keithly 485 Picoammeter records the photodiode current.

The fiber output of the isolator now interfaces with a pin photodiode. Reverse bias of $5 \mathrm{~V}$ is applied to the device, with a Keithley picoammeter connected in 


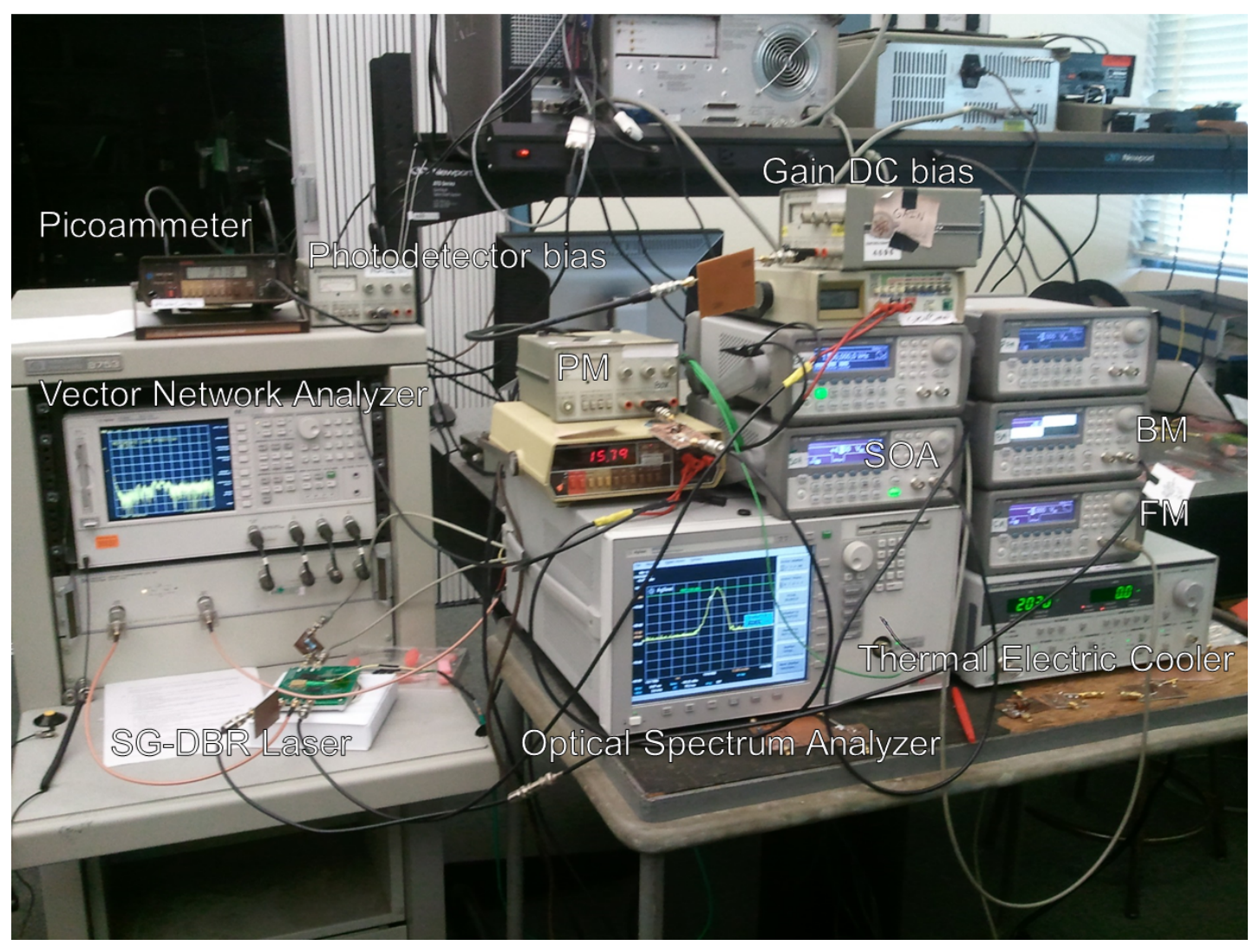

Figure 6.2: Amplitude Modulation (AM) Experimental Test Setup Photograph.

series to measure the photodiode current. Coaxial cables are used for all bias and ammeter connections to mitigate any noise induced error on the photodiode readings. The chassis of the detector is grounded to the bias supply. An SMA cable is connected from the output of the detector into port 2 of the particular VNA used (Anritsu MS4622A). The VNA is configured to provide a $0 \mathrm{dBm}$ stimulus from $10 \mathrm{MHz}-3 \mathrm{GHz}$ on port 1. This sinusoidal sweep of constant power provides the necessary amplitude modulation for the gain and SOA sections. The overall premise is to electrically modulate the laser from port 1, produce optical power fluctuations from the laser, receive and convert the optical to electrical using the photodiode, and bring the electrical signal back into port 2 of the VNA. 
Selecting a $S_{21}$ magnitude plot on the VNA provides the modulation transfer function of the laser at a particular bias current.

If available, a lightwave component analyzer could also be used to obtain the modulation response of the laser. It works similarly to a VNA, except it includes calibrated optical sources and receivers to perform s-parameter like measurements, and is more suited for optical measurements. However for this simple test, a VNA and an external photodetector are the only elements required to obtain the conversion efficiency of the laser with respect to modulation frequency as detailed in figure 6.1. By investigating several bias currents for the gain and SOA sections the optical bias conditions for maximum frequency response can be determined.

\subsection{AM Response on GAIN Section}

The gain section is the primary source of the laser's optical output, physically located between the two primary mirrors of the cavity. Several cases of DC biasing ranging from $35.82-140 \mathrm{~mA}$ are injected into the DC biasing port of the VNA to observe the efficiency. At low biasing currents, such as $35.82 \mathrm{~mA}$ in figure 6.3, the laser has not sufficiently breached the threshold level. Recall that when below threshold the spontaneous emission dominates over stimulated emission, indicating a relatively poor frequency response due to domination by minority carrier lifetimes for $35.82 \mathrm{~mA}$. For all cases of gain bias testing the SOA is fixed at $98 \mathrm{~mA}$, with a temperature lock of $20^{\circ} \mathrm{C}$ for the TEC.

The threshold current of the laser is experimentally determined to be approximately $58 \mathrm{~mA}$ in figure 6.4. Notice for this case above threshold the rolloff is visible in frequency response, characteristic of moderate biasing currents above 


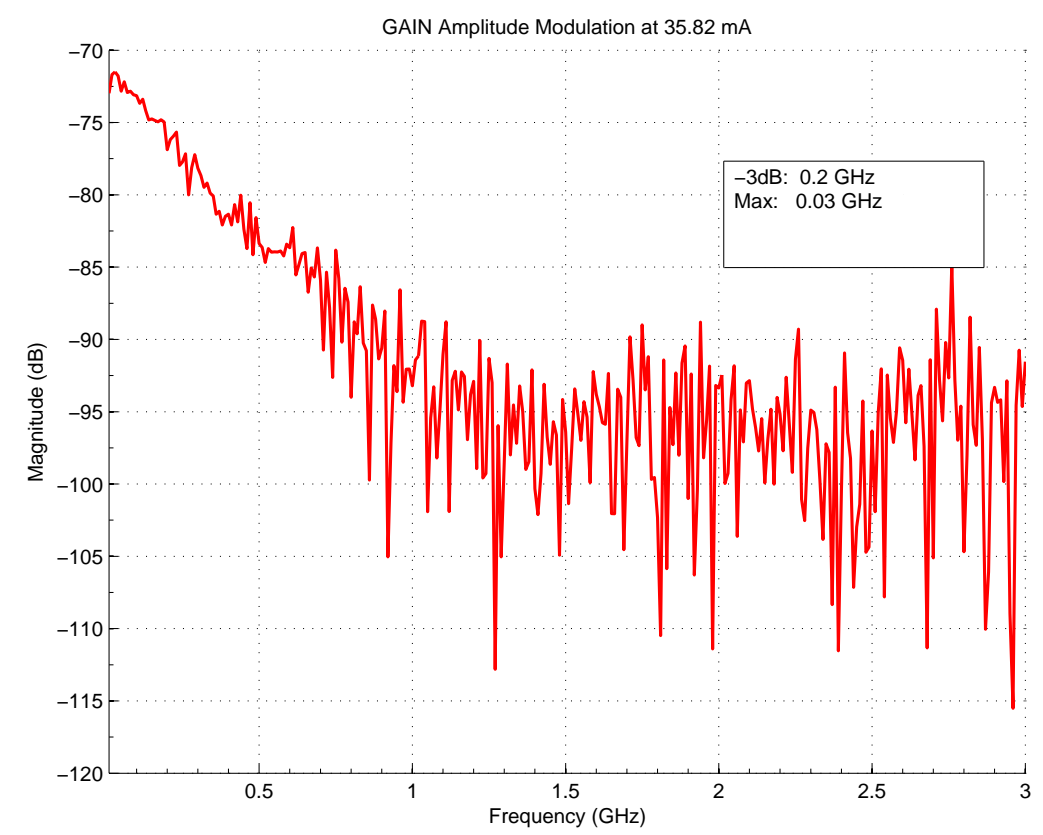

Figure 6.3: Gain Section AM Response at $35.82 \mathrm{~mA}$ Bias. Laser is biased below threshold. Modulation bandwidth of approximately 200 $\mathrm{MHz}$.

threshold. Calculating the cutoff frequency, $-3 \mathrm{~dB}$, for this current yields a modulation bandwidth of $930 \mathrm{MHz}$. Comparing to the previous bias current recorded at $57.10 \mathrm{~mA}$ produced only $340 \mathrm{MHz}$, indicating the threshold is likely between both currents.

With further increases in the biasing current the effective bandwidth generally increases. The relaxation oscillation, which is the natural resonance of the laser, begins to shift to higher frequencies with additional bias [25]. This oscillation resonance is clearly visible in bias cases starting at $61.9 \mathrm{~mA}$ in figure 6.5. Notice the global maximum appearing at $1.19 \mathrm{GHz}$, indicating the resonance oscillation peak. Increasing the current to $66.5 \mathrm{~mA}$ (figure E.1m) further expands the peak to $1.70 \mathrm{GHz}$, and even further so at $1.97 \mathrm{GHz}$ with $68.7 \mathrm{~mA}$ (figure E.1n). A full summary of all bias conditions tested is presented in appendix E. Typically with 


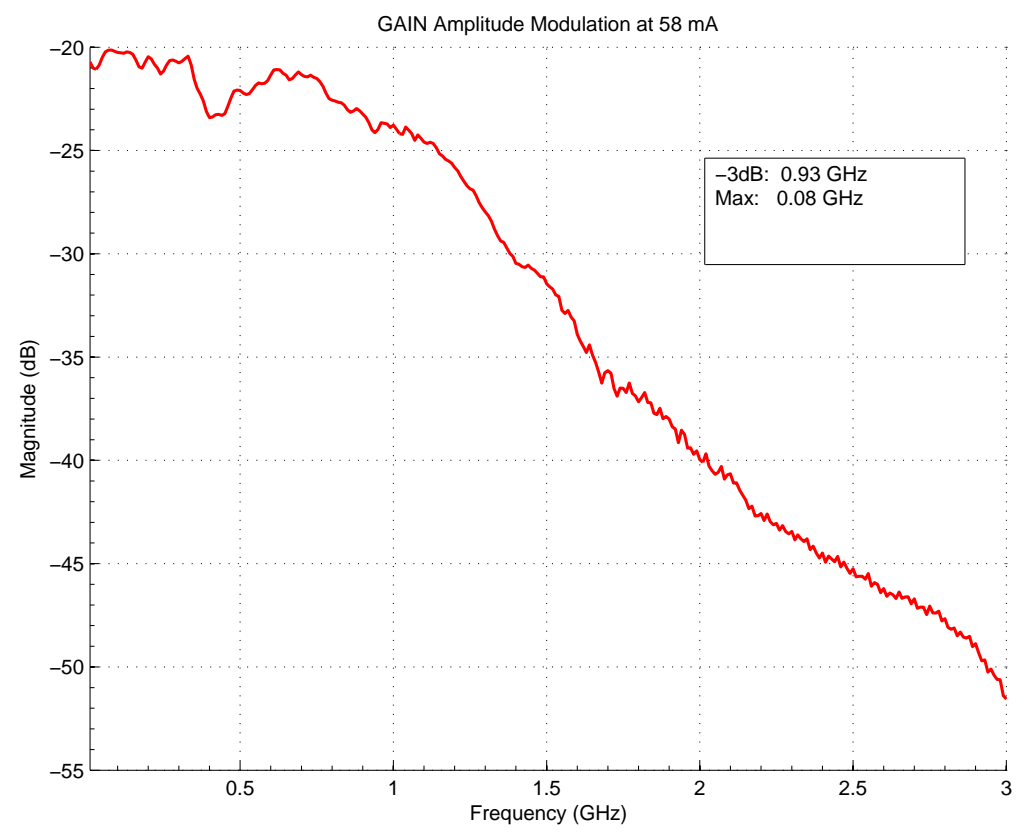

Figure 6.4: Gain Section AM Response at $58.00 \mathrm{~mA}$ Bias. Laser is biased above threshold. Modulation bandwidth of approximately 930 MHz.

DFB or DBR based lasers, as the bias is increased not only will the bandwidth increase, but the responsivity as well until a particular point where beyond this high frequency response degrades.

Figure 6.6 details a bar graph plotting the $-3 \mathrm{~dB}$ modulation frequency with respect to bias current. According to the plot, the maximum bandwidth of 2.69 GHz occurs at approximately $95 \mathrm{~mA}$. Bias currents of 80-100 mA provide optimal bandwidths at approximately $2 \mathrm{GHz}$.

The intensity modulation response also peaks at the relaxation oscillation frequency, known as $f_{p}[26] . f_{p}$ is dependent on the resonance between electrons and photons in the active region as well as the damping frequency. According to theory [26], the relaxation oscillation frequency increases proportionally to the square of the input bias current. After the oscillation peaks, as in figure 6.5 


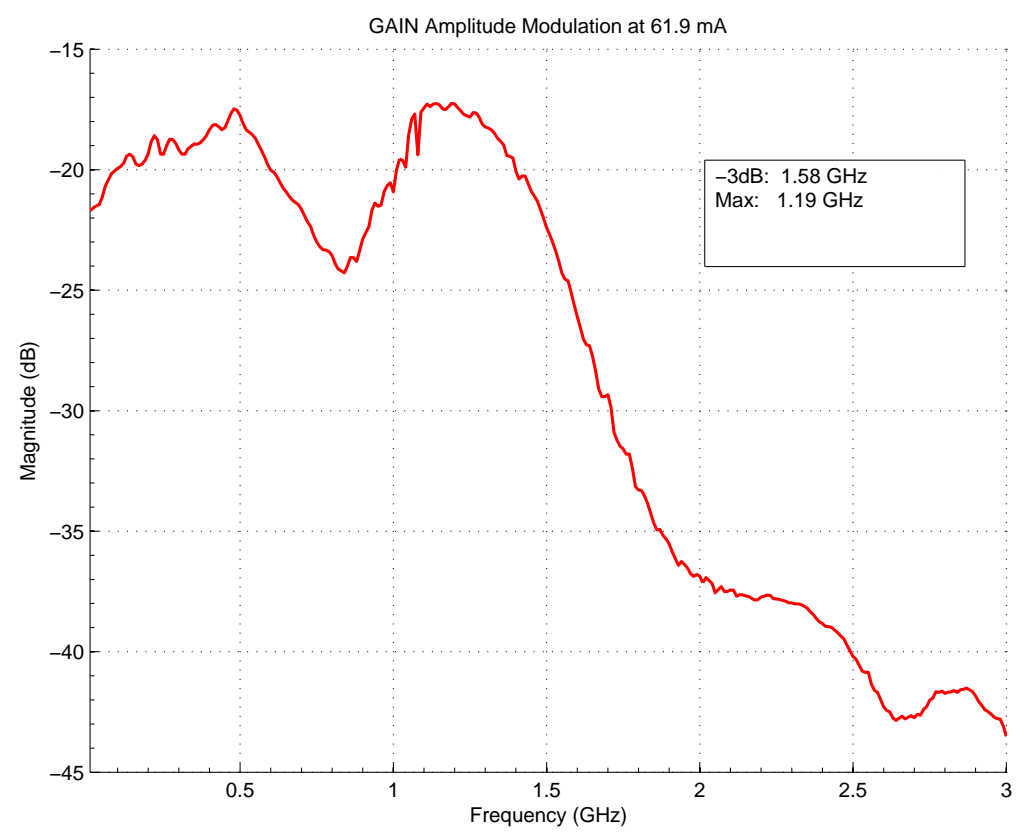

Figure 6.5: Gain Section AM Response at $61.90 \mathrm{~mA}$ Bias. Relaxation oscillation is present, as indicated by the peaking at $1.19 \mathrm{GHz}$. Modulation bandwidth of approximately $800 \mathrm{MHz}$.

a steep roll off is present, at approximately $12 \mathrm{~dB}$ per octave, consistent with theory.

Plotting the maximum peak of each bias case, as in figure 6.7 provides insight to the resonance of the laser cavity with respect to bias injection current. From $80-110 \mathrm{~mA}$, a generally increasing trend is observed by the shift of the global maximum. In these cases the peak is evident of the relaxation oscillation frequency.

It is important to note that the relaxation oscillation frequency is not the only limiting factor for the modulation bandwidth. The electrical parasitics of the device, including the previously mentioned wirebonds, junction capacitance of the diodes, and ohmic resistance of the leads all contribute to limiting the bandwidth. In general, it is evident that lower biasing currents the modulation 


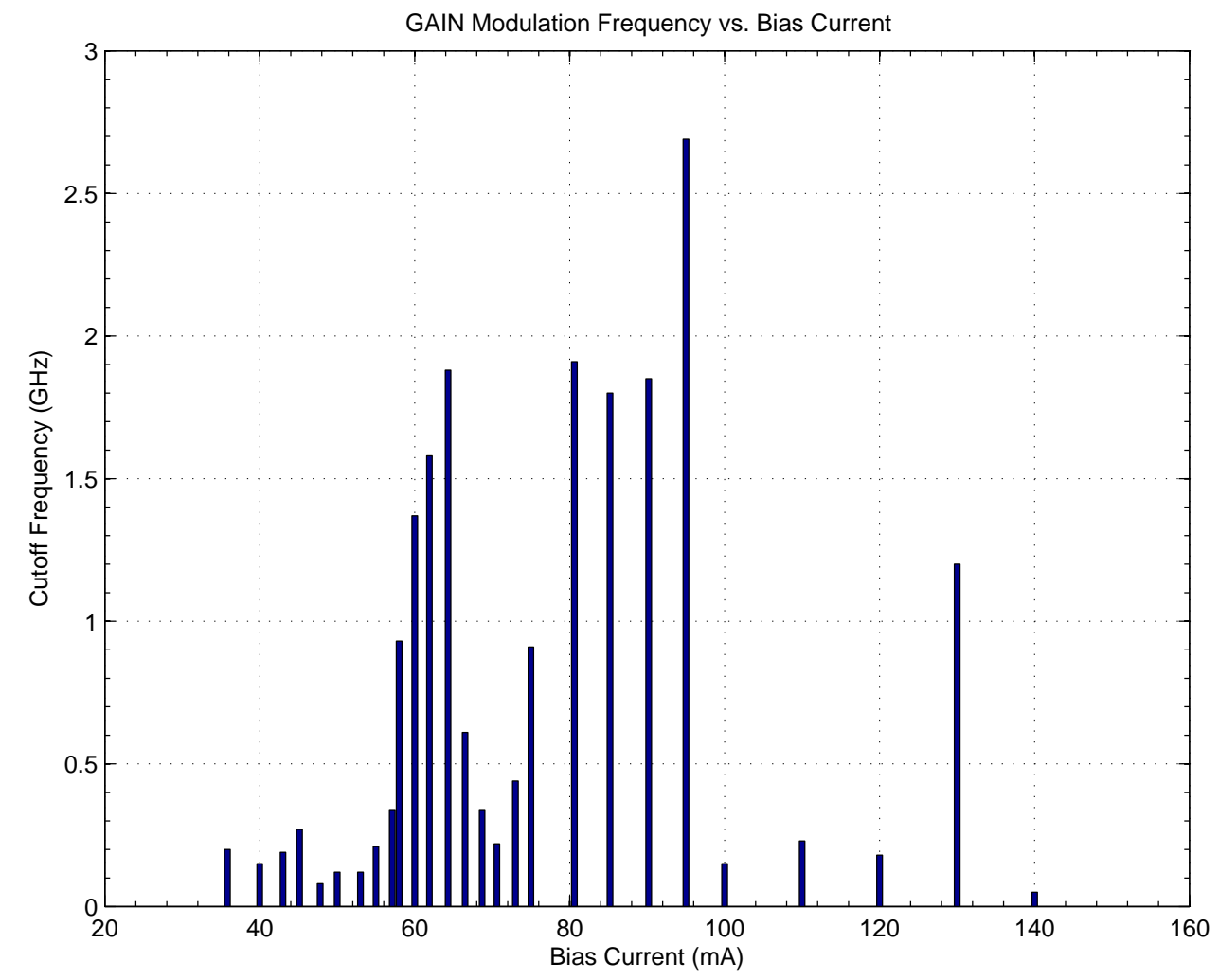

Figure 6.6: Gain Section AM Response Summary. The modulation frequency plotted with respect to Gain bias current. Fixed bias for all ports with SOA $=98 \mathrm{~mA}$. Optimal bandwidth is achieved with bias of 80-100 mA, providing greater than $2 \mathrm{GHz}$ bandwidth. Notice the dip occurring at approximately $70 \mathrm{~mA}$, this is likely evident of the RC limitation transitioning over to relaxation limitations on the bandwidth for larger currents.

bandwidth is restricted by the device parasitics, however for higher bias currents the relaxation oscillations become more prominent as the limiting factor. 


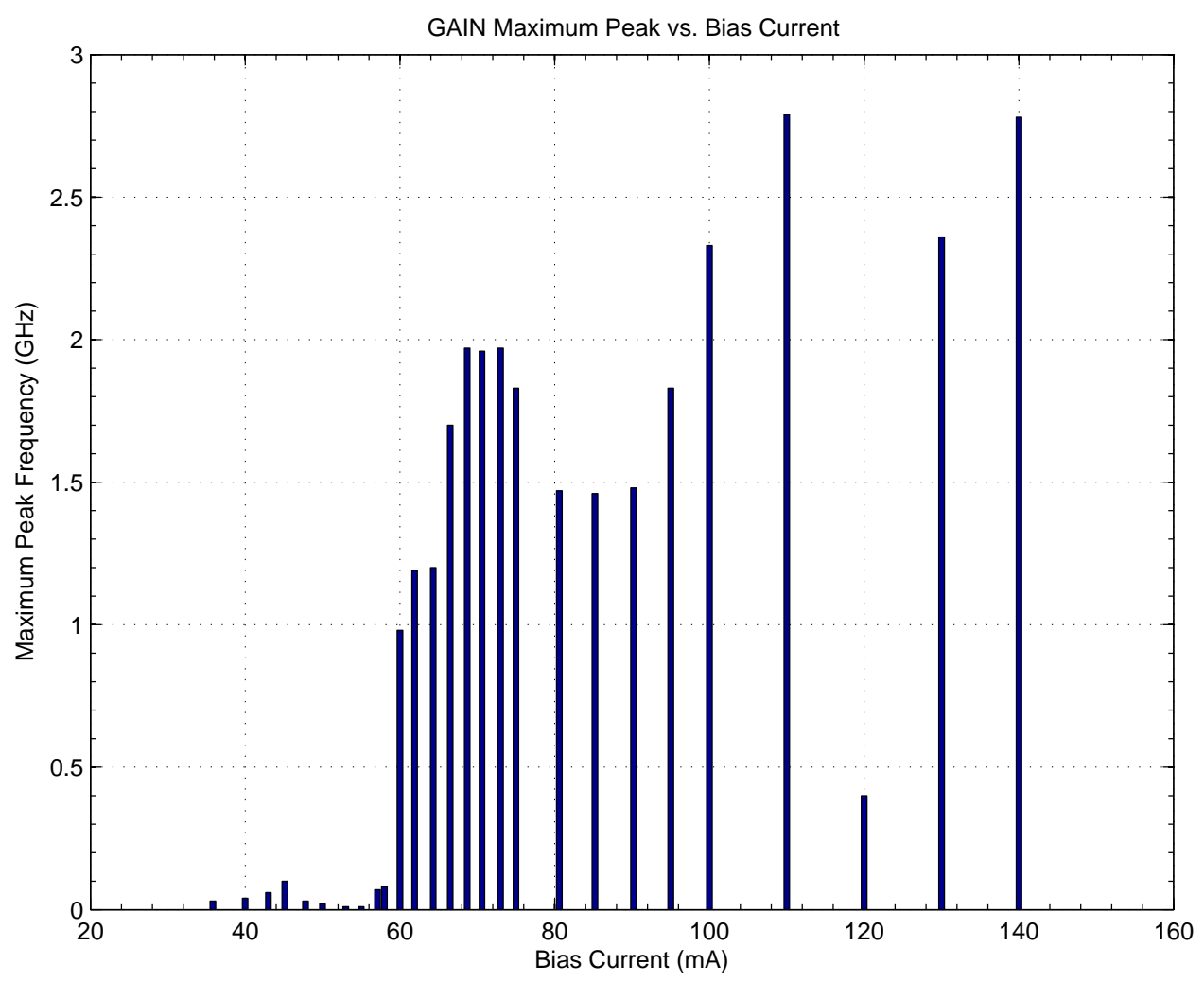

Figure 6.7: Gain Section AM Response Relaxation Oscillation Summary. The maximum peak frequency, generally corresponding to the relaxation oscillation frequency, with respect to Gain bias current. Fixed bias for all ports with SOA $=98 \mathrm{~mA}$. The relaxation peak is consistent with the square law, proportionally increasing with the square of the bias current. 


\subsection{AM Response on SOA Section}

The SOA, unlike the Gain section, is located outside of the main laser cavity. It's primary purpose is to act as an additional inline amplifier for the optical output. Essentially the SOA is a mirrorless laser, with the mirrors replaced with anti-reflective coatings [27]. As performed previously with the gain section, the SOA is DC biased and amplitude modulated with the gain fixed at $100 \mathrm{~mA}$. FM, $\mathrm{BM}$, and PM are set to zero bias for all tests.

Due to the fact that the laser with the gain section biased above threshold is already lasing, biasing the SOA section does not carry as great of a dependence to modulation frequency as before with the gain section. Even at low bias values of $10 \mathrm{~mA}$ in figure 6.8, a relatively high bandwidth of $280 \mathrm{MHz}$ is achieved. Further increasing bias current extends the operating bandwidth until a plateau is reached of approximately $500 \mathrm{MHz}$ in the range of $50.50-150.0 \mathrm{~mA}$ bias. The particular bias chosen for most of the experiments is $98 \mathrm{~mA}$, yielding a SOA modulation response resembling the $100 \mathrm{~mA}$ case of figure 6.9, with an effective bandwidth of $510 \mathrm{MHz}$.

A full summary, similar to the figure in the gain section, is presented in figure 6.10 detailing the measured modulation bandwidth with respect to SOA bias current. Again, notice the maximum effective modulation frequency of 450$500 \mathrm{MHz}$ for bias greater than $50 \mathrm{~mA}$. Figure E.2 in appendix E presents several $S_{21}$ plots for bias cases from $0-168 \mathrm{~mA}$ in a similar manner to the gain sections. 


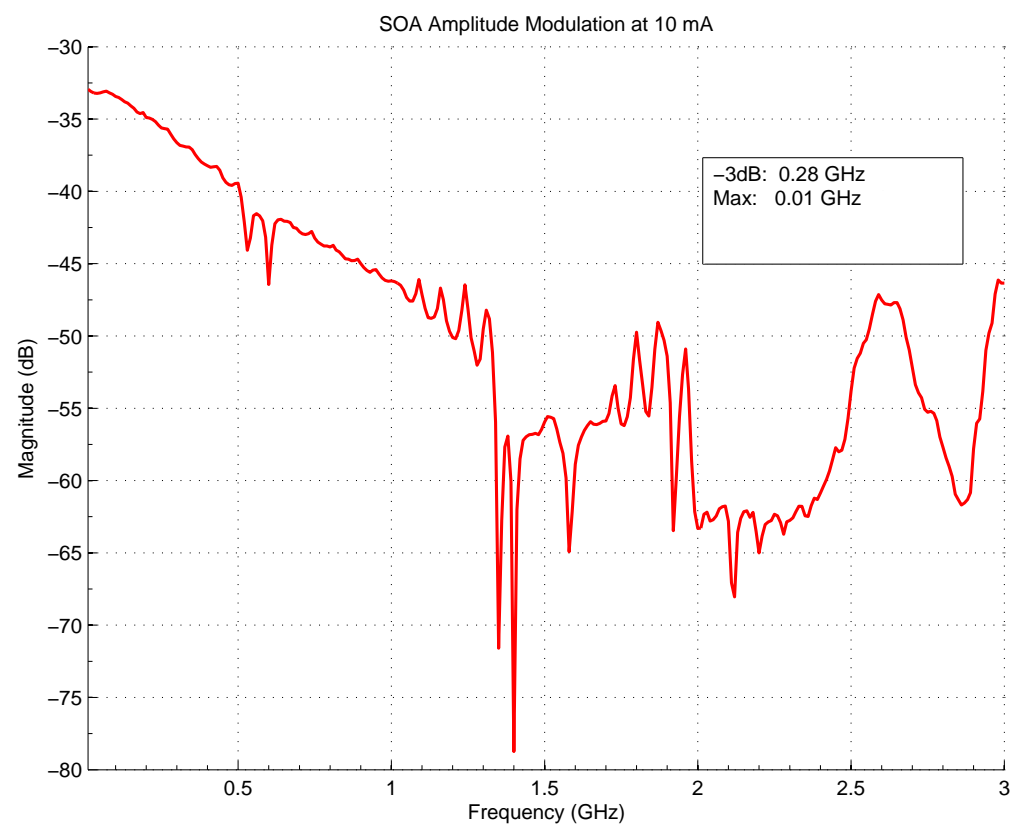

Figure 6.8: SOA AM at $10.00 \mathrm{~mA}$.



Figure 6.9: SOA Section AM Response at $100.00 \mathrm{~mA}$ Bias. $I_{F M}=I_{B M}$ $=I_{P M}=0 \mathrm{~mA}, I_{\text {Gain }}=100 \mathrm{~mA}$. Modulation bandwidth of approximately $510 \mathrm{MHz}$. 


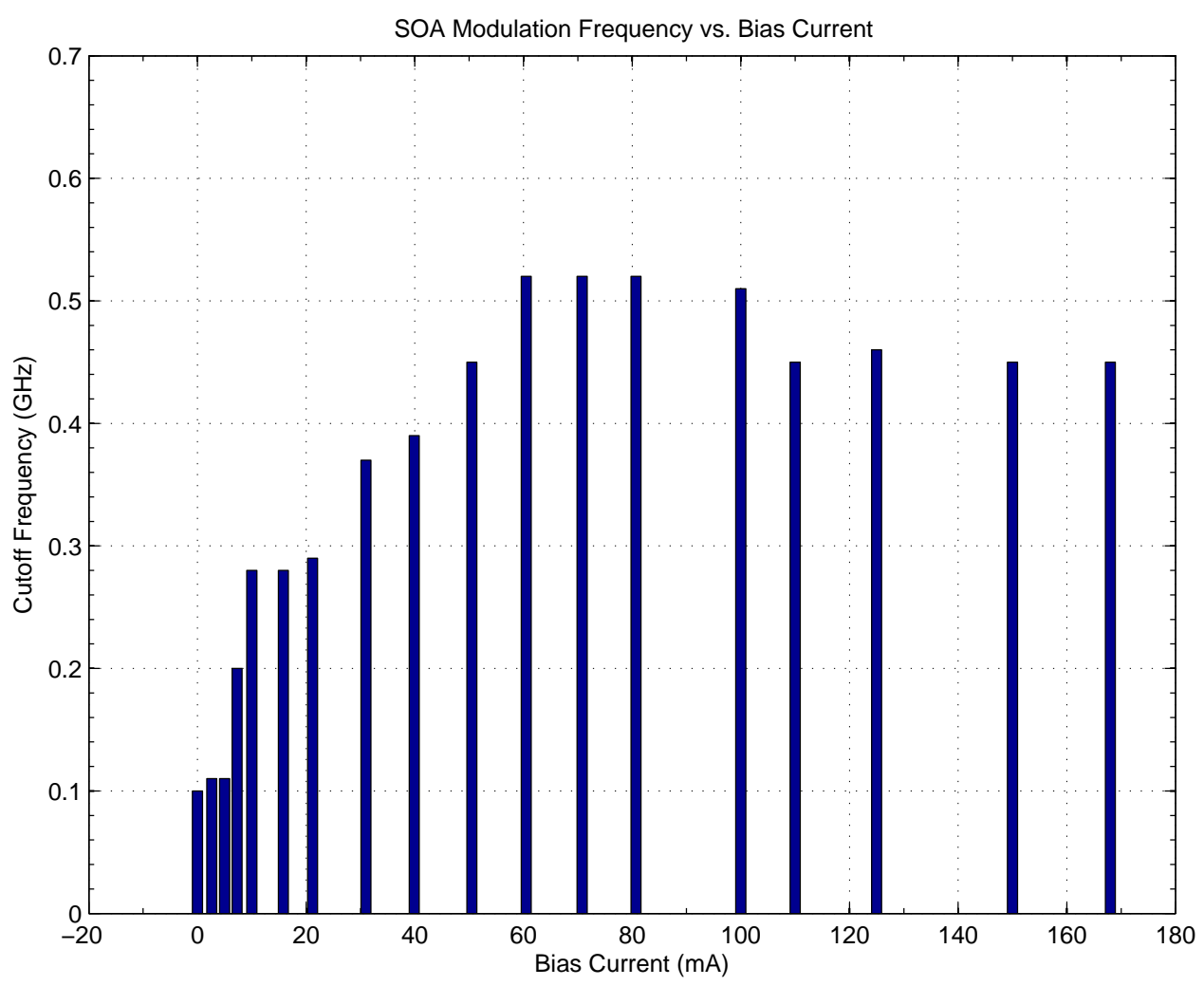

Figure 6.10: SOA Section AM Response Summary. The modulation frequency with respect to varying SOA bias current only is plotted. $I_{F M}=I_{B M}=I_{P M}=0 \mathrm{~mA}, I_{\text {Gain }}=100 \mathrm{~mA}$. VNA output power $=0$ $\mathrm{dBm}$. Optimal bias on SOA section to achieve greater than $500 \mathrm{MHz}$ modulation bandwidth is $50-150 \mathrm{~mA}$. 


\section{Chapter 7}

\section{Frequency Modulation (FM) Bandwidth Characterization}

In the previous chapter the amplitude modulation response for the amplifier sections was characterized to determine the maximum frequency at which the intensity can be modulated. As mentioned previously for wavelength tuning, the front, back, and phase sections control the cavity size and therefore resonant frequency. Modulating these three sections in a precise and controlled manner permits the linear frequency ramp required for SS-OCT. The sweep repetition rate in $\mathrm{OCT}$, thereby the imaging acquisition time, is limited by the maximum frequency modulation rate of the SG-DBR mirror sections. Characterization of the performance is critical to determine the behavior and limits of operation.

\subsection{Test Setup}

Intensity measurements are relativity easy, requiring a simple photodetector as the receiver and the VNA to provide the input modulation, detailed in 
chapter 6. However to appreciate the frequency dynamics of the SG-DBR, a more involved measurement process is required. Only simple power or intensity fluctuations are observable on a typical photodetector. In order to quantify the modulated frequency and phase, optical interferometric techniques must be employed [25].

An introduction to the basic theory behind interferometers and the laser spectrum is necessary before delving into the experimental setup. For all of the frequency and spectrum based tests the laser must be operating in a singlelongitudinal mode (SLM). Recall back to figure 3.7 with the single mirror tuning, in part $\mathrm{f}$ exists the non-ideal case of multiple modes resonating and therefore is not SLM. When manually tuning the injection currents it is critical for there to exist a SLM with the greatest possible side mode suppression ratio (SMSR) to allow for the highest signal integrity.

A laser's output spectrum consists of the primary SLM, containing the majority of the power, in a narrow Lorentzian shaped peak as illustrated in figure 7.1 [6]. Small sidebands are present displaced approximately $10 \mathrm{GHz}$ on either side of the primary peak, caused by relaxation oscillations. Further away in the spectrum exists the secondary sidemodes, also known as mirror modes. They are located approximately $150 \mathrm{GHz}$ from the central frequency, $\nu$. Finally the floor of the figure is primarily consisted of spontaneous emission, the precursor to lasing.

Interferometric type measurements, such as the methods employed to study frequency response, operate on the principle of superposition. Consider when two waves of similar frequency combine together, only differing by their phases. The output consists of constructive interference when in phase, and destructive interference when out of phase. Two similar categories exist for interference measurements - heterodyne and homodyne. In the heterodyne case, a local 


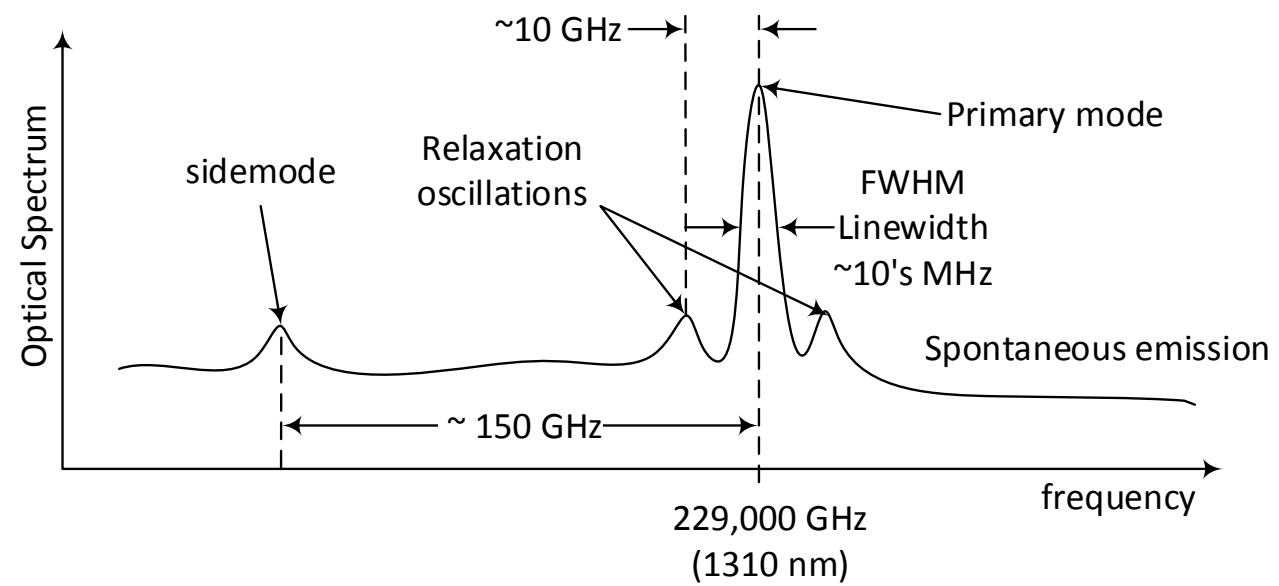

Figure 7.1: Laser Spectrum. The primary peak at $1310 \mathrm{~nm}(229,000$ $\mathrm{GHz}$ ) for this example contains small relaxation oscillations on either side. Secondary modes caused by mirrors are offset $\sim 150 \mathrm{GHz}$ from the central peak. The floor is consistent of amplified spontaneous emission. Figure modeled after Ref. [25]

oscillator (LO) is utilized as a reference source to observe the source laser under test (SLUT). Both signals combine through the use of an optical coupler with the resulting pattern observed on a spectrum analyzer. From the photodetector's point of view, if each signal were viewed separately, only the intensity would be observed, with no phase information carried over. This is due to the fact that electronic equipment cannot respond fast enough to the several hundred terahertz frequencies of light, and why direct detection of frequency is not possible, necessitating the use of interferometric methods. The LO is set to a slightly lower frequency than the SLUT. The spectrum consists of the sum and the difference of the two sources, each known as heterodynes. Unfortunately with the heterodyne case, the requirement of a second LO laser adds increased cost and complexity to the measurement.

The second case, and the method utilized in this paper, is optical homodyne 
detection. A Mach-Zehnder implementation of this measurement consists a single laser as the input source feeding into an optical power splitter, shown in figure 7.2. The $3 \mathrm{~dB}$ splitter launches into two separate fiber paths. One is a reference path, and the other longer path causes an inherent differential time delay, $\tau_{0}$, of the input signal. Consider the input field $E_{s}$ from the source laser traversing down both arms of the interferometer. The power of the source $P(t)=\left|E_{s}(t)\right|^{2}$ is split into each arm. The resulting power delivered from each arm to the photodetector, $P_{1}(t)$ and $P_{2}(t)$, generates a photocurrent

$$
i(t)=\mathcal{R}\left[P_{1}(t)+P_{2}(t)+2 \sqrt{P_{1} P_{2}} \cos \left(2 \pi \nu_{0} \tau_{0}+\Delta \phi(t)\right)\right]
$$

with the average phase of the interferometer given by $2 \pi \nu_{0} \tau_{0}$, where $\nu_{0}$ is the average frequency, $\tau_{0}$ is the delay, and $\mathcal{R}$ is the detector responsivity. The resulting phase difference is represented by $\Delta \phi\left(t, \tau_{0}\right)=\phi(t)-\phi\left(t-\tau_{0}\right)$, from the frequency modulation of the input signal. Figure 7.3 shows the output photocurrent with respect to frequency. Notice the period of the beating frequency is the interferometer delay $\tau_{0}$, which is the reciprocal of the free-spectral range (FSR). The FSR related by the difference in frequency such that a phase shift of $2 \pi$ exists between the combination of the two fields from the interferometer arms. The quadrature point in figure 7.3 occurs at $\pi / 2$ multiples.

The quadrature point allows for small phase modulation to be linearly converted into intensity changes. This is the essence of the optical FM to AM converter, also known as a frequency discriminator. For the discriminator to be valid it requires $\Delta \phi\left(t, \tau_{0}\right)$ to be small. According to the small angle approximation $\left(\sin \left(\Delta \phi\left(t, \tau_{0}\right)\right) \approx \Delta \phi\left(t, \tau_{0}\right)\right.$, the interferometer causes linear conversion of frequency modulation into power fluctuations in quadrature bias. Modifying 
equation 7.1, taking into account the approximation, yields

$$
i(t)=\mathcal{R}\left[P_{1}(t)+P_{2}(t)+2 \sqrt{P_{1} P_{2}} 2 \pi \nu_{0} \tau_{0}+\Delta \phi(t)\right]
$$

The $2 \pi \nu_{0} \tau_{0}$ from equation 7.1 is removed due to the quadrature requirement $(\pi / 2)$ modifying the cosine term into the sine term, and the approximation eliminating the sine operation. The powers $P_{1}(t)$ and $P_{2}(t)$ are the only components detectable via direct detection, with the final term containing the interference signal used in FM response measurements of this chapter.

The FSR of the interferometer determines the frequency of the spacing, and thus the precision required for obtaining a quadrature biased lock. Recall the relation for the frequency $f$ and wavelength $\lambda$ to the speed of light $c$

$$
c=f \lambda
$$

Rearranging, and taking the $\Delta$ of each side yields equation 7.4. Continuing minor algebraic manipulation in equations $7.4-7.7$ yields the form relating the interferometer wavelength difference $\Delta \lambda$ to the frequency difference $\Delta f$, known as the FSR in figure 7.3. With the assumption $\lambda_{1} \approx \lambda_{2}$ for the center wavelength, equation 7.7 is simplified to 7.8 [25].

$$
\begin{gathered}
\Delta f=\Delta\left(\frac{c}{\lambda}\right) \\
\Delta f=\frac{c}{\lambda_{2}}-\frac{c}{\lambda_{1}}
\end{gathered}
$$




$$
\begin{gathered}
\Delta f=\frac{c \lambda_{1}-c \lambda_{2}}{\lambda_{2} \lambda_{1}} \\
\Delta f=\Delta \lambda \frac{-c}{\lambda_{2} \lambda_{1}} \\
\Delta f=\Delta \lambda \frac{-c}{\lambda^{2}}
\end{gathered}
$$

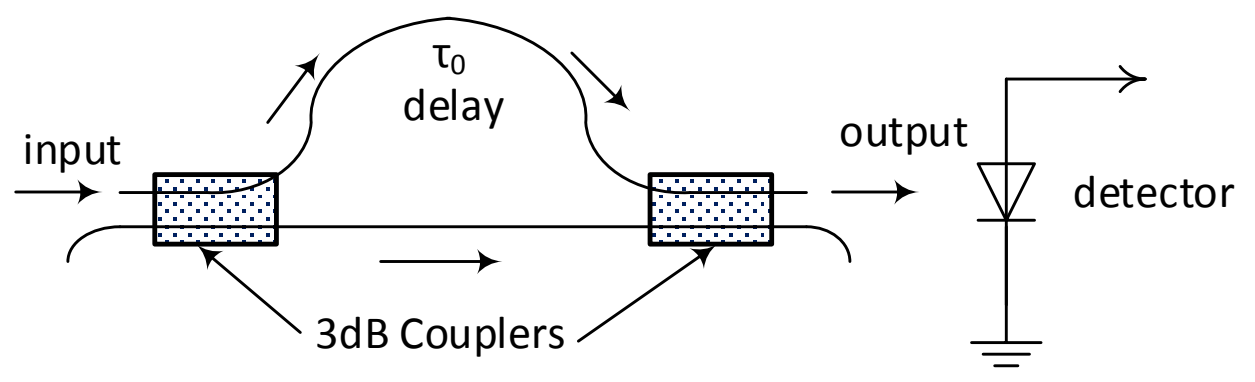

Figure 7.2: Mach-Zehnder Interferometer Setup. Input power is split via coupler into two paths - one with time delay $\tau_{0}$ and reference. The signals recombine to produce a photocurrent at the output on a photodetector to monitor on an ESA or VNA.

For a Mach-Zehnder Interferometer (MZI) operating at $1310 \mathrm{~nm}$ with $\Delta \lambda=$ $100 \mathrm{pm}$, the FSR $\Delta f=17.5 \mathrm{GHz}$. All measurements for the phase, front, and back sections utilize the 100 pm MZI for the discriminator.

The experimental testing arrangement is shown in figure 7.4. The FM tests are performed on each of the frequency controlled sections in separate tests. The optical output of the SG-DBR is once again fed through an optical isolator into an interferometer. There are two outputs from the interferometer, each out of phase $180^{\circ}$ to each other. One port is fed directly into the biased photodetector, and into the port 2 of the VNA. The other output is fed into the OSA. Primarily 


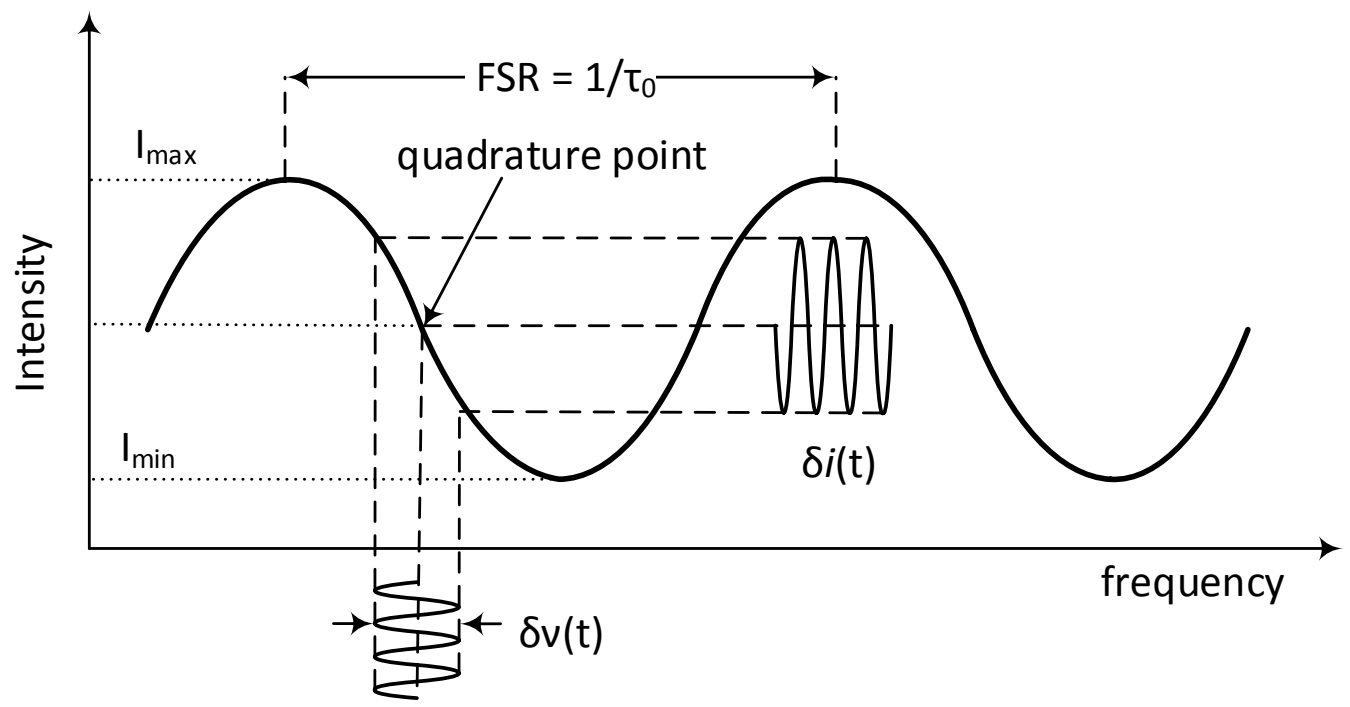

Figure 7.3: Mach-Zehnder Interferometer Output. When biased in quadrature, an input $\delta \nu(t)$ of small frequency linearly converts into amplitude changes $\delta i(t)$, observable on the photodetector. The quadrature bias point is located midway between the maximum and minimum intensity values. Free Spectral Range (FSR) is based upon the differential time delay of the interferometer.

the OSA is used to simultaneously monitor the output spectrum to validate a SLM exists during manual tuning.

The VNA is DC biased, with current limiting protection, into port 1 for the section under test with a stable linear power supply to mitigate injected noise. Port 1 is directly connected to the particular section. The VNA is configured to display a $S_{21}$ magnitude plot relating the input to output. A high level overview consists of injecting constant power swept sinusoid stimulus into the frequency dependent sections (front, back, and phase), in turn frequency modulating the laser's optical output. Finally the use of an FM discriminator converts frequency to amplitude modulation for observation on a photodetector, converting into 
electrical signals back into the return port of the VNA, thus plotting the transfer function of the port under test.

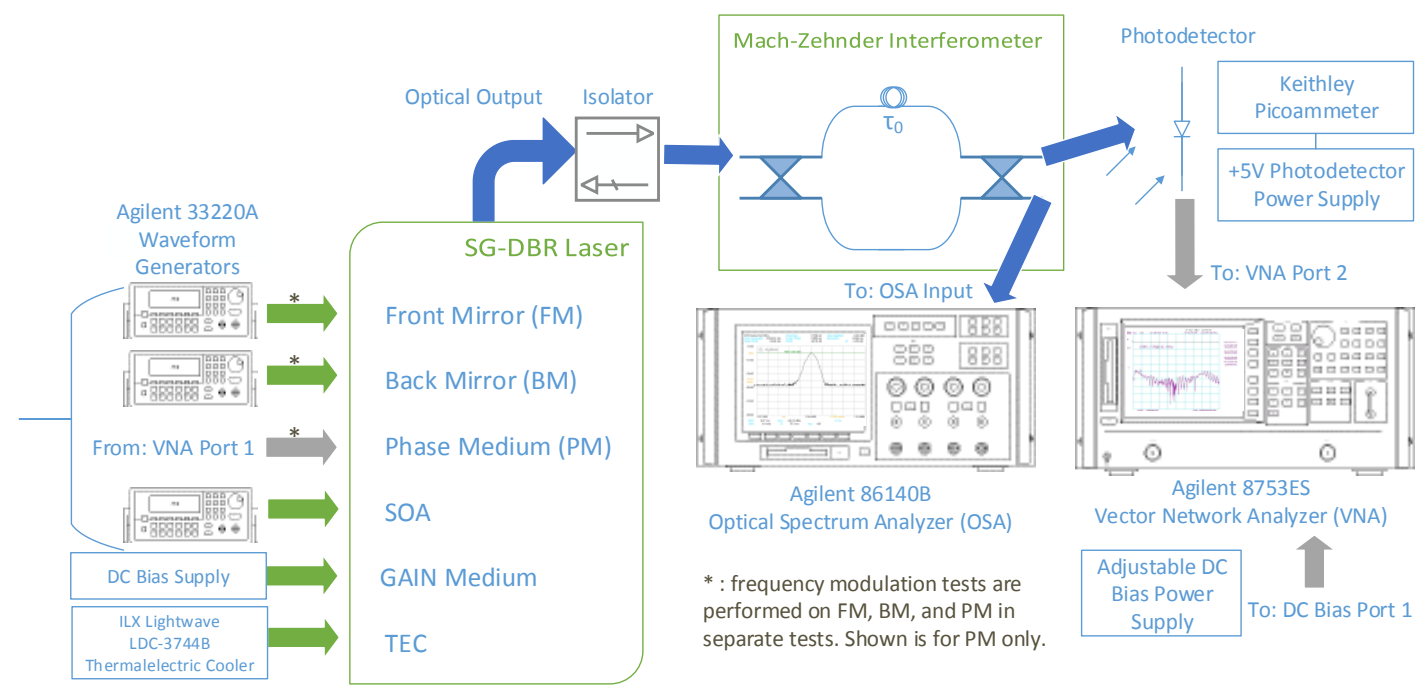

Figure 7.4: Swept Sinusoid Frequency Modulation (FM) Experimental Test Setup. DC electrical lines are denoted with green arrows, SMA based coaxial connections with gray arrows, and fiber optical links with blue arrows. Interferometer FSR $=17.5 \mathrm{GHz}(100 \mathrm{pm}$ at $1310 \mathrm{~nm})$.

The measurement characterizes both AM and FM responses simultaneously. Unfortunately AM signals still exist even when perfectly biased in quadrature and can distort the FM measurements. Two methods can be employed to differentiate the response types. The first method requires an active quadrature locking and control circuit. This maintains quadrature lock on the positive and negative slopes of the interferogram. Recording the complex amplitude and phase at both quadrature polarities is necessary. AM signals are in phase for both quadrature sets, while the FM signals are $180^{\circ}$ out of phase. Therefore only the AM response is obtained by adding both sets, and the FM response by subtracting the negative from positive lock [28].

However through a careful understanding of the signal and expectations, a 
decent approximation for the FM response can be extracted with a reduced complexity setup. The second method, utilized by this measurement, involves manually tuning the frequency of the laser to align it at quadrature and several key points as shown in figure 7.5 and described in detail in section 7.2. 


\subsection{FM Response on Phase Section}

The phase section is utilized for fine incremental wavelength tuning, and thus is one of the more sensitive to frequency modulation. Obtaining the maximum modulation frequency is necessary to define limits for the sweep rate. The interferogram in figure 7.5 shows several essential points overlaid on the output of the MZI. As the bias to the phase section is changed it results in a corresponding change in frequency of the laser (tuning). Tuning the laser causes the input stimulus from the VNA to be applied at various locations on the MZI curve. Recall that for the FM to AM conversion to be applicable the linear portion must be utilized, known as the quadrature point. By sweeping through various points in the curve a detailed characterization of the AM and FM response can be obtained.

Point A of figure 7.5 is present on the peak of the curve, indicating maximum photodetector current and therefore also maximum AM response, while containing low FM response levels [29]. Moving down the curve, the AM level will decrease as will photocurrent, however FM response will increase. FM is at maximum when in quadrature, also photodetector current is ideally half of

the maximum obtained from point A. Further traversing the curve to point $\mathrm{C}$ reaches a minimum for photocurrent, with minimums for both AM and FM response. Point D rises in FM response, with AM still present, at the second quadrature lock. The full period of the MZI curve completes back to another photocurrent and AM maximum at point E. Using this criterion a simple decision matrix is established to verify the location on the curve for various bias captures, summarized in table 7.1.

Manually tuning throughout the MZI curve is a highly iterative process. It begins with viewing the OSA for a stable SLM, with even sideband distribution 
and a high SMSR. Adjustment of the front and back mirrors are used to achieve a stable SLM, with the phase to fine tune. Once a basic SLM is achieved, the OSA display is zoomed in at $0.2 \mathrm{~nm}$ per division to allow for tracking the peak wavelength more accurately. With the particular $17.5 \mathrm{GHz}(100 \mathrm{pm})$ interferometer $0.1 \mathrm{~nm}$ of contiguous tuning is required before mode hopping occurs between the peaks. Adjusting the phase bias injection current causes fine tuning of the peak. Increments of approximately $200 \mu A$ are adjusting manually for the phase, while constantly observing the OSA display for mode hops or unstable modes. For each data set the current for every section, photodetector current minimum and maximum, primary wavelength peak, and VNA $S_{21}$ characteristics are recorded. A combination of bias currents are adjusted to maintain the continuous frequency sweep and thereby track throughout the different key points in the MZI curve.

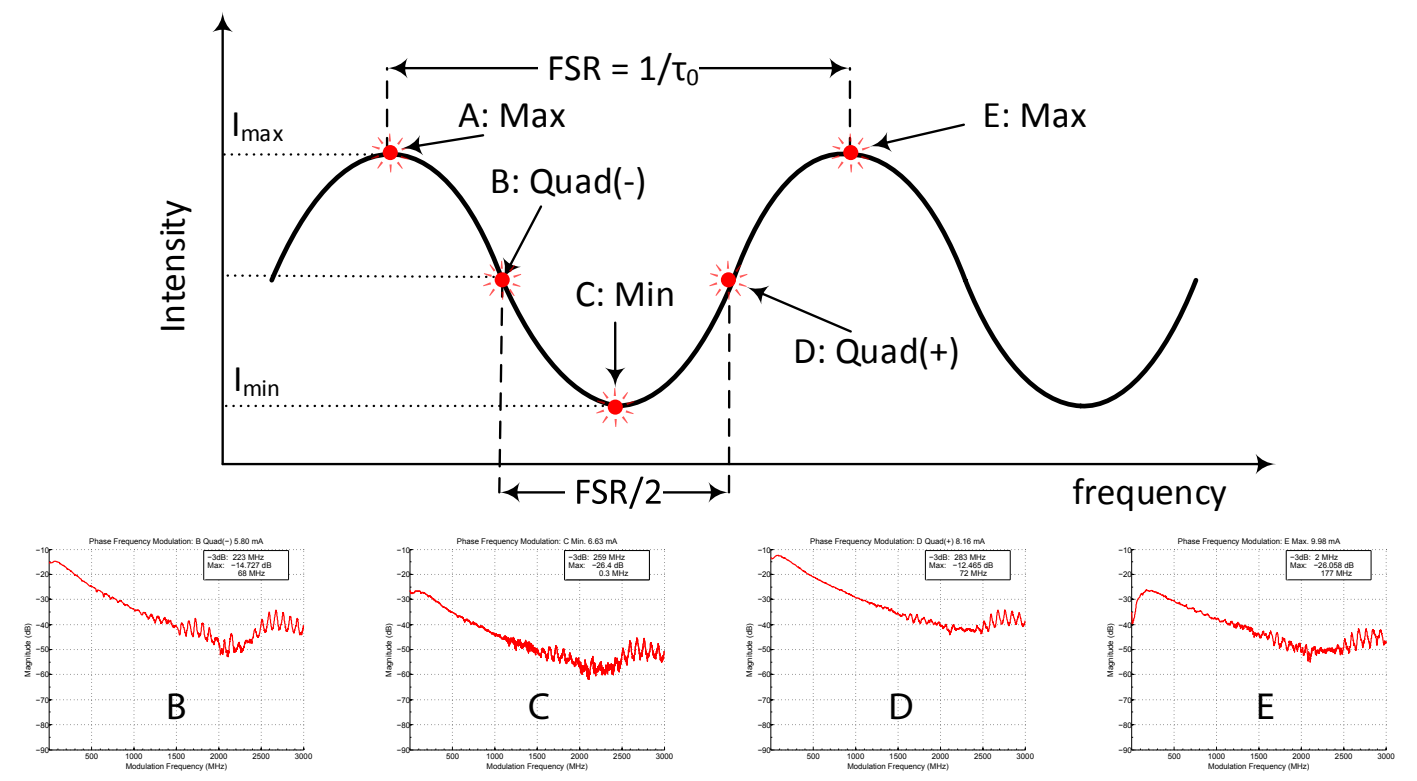

Figure 7.5: Phase Frequency Modulation Data. Critical points on the interferometer output: (B) Negative quadrature lock; (C) Minimum average amplitude; (D) Positive quadrature lock; (A) \& (E) Maximum average amplitude. 
Table 7.1: Quadrature Decision Matrix. Quadrature is defined in points $\mathrm{B}$ and $\mathrm{D}$ where photocurrent $I_{P D}$ is half, $\mathrm{AM}$ signal is reduced, and FM signal is at maximum. Refer to figure 7.5 for point locations on the MZI output example.

\begin{tabular}{|r||r|r|r|r|}
\hline Case & Point A(E) & Point B & Point C & Point D \\
\hline \hline$I_{P D}$ & $\max$ & $1 / 2$ & $\min$ & $1 / 2$ \\
\hline $\mathrm{AM}$ & $\max$ & $1 / 2$ & $\min$ & $1 / 2$ \\
\hline $\mathrm{FM}$ & $\min$ & $\max$ & $\min$ & $\max$ \\
\hline
\end{tabular}

Magnified plots from each case represented in figure 7.5 are presented in the following figures 7.6-7.9. Since points A and E are separated by the FSR period, responses at each point are nearly identical, and only point $\mathrm{E}$ in figure 7.6 is shown. The picoammeter connected to the photodetector monitors the current range, which is at a maximum of approximately $0.89-1.71 \mathrm{~mA}$, the the phase biased at $9.98 \mathrm{~mA}$. The magnitude of the FM response is approximately $-35 \mathrm{~dB}$ at $300 \mathrm{KHz}$, towards the lower range of responses, agreeing with the assumptions for minimal FM at the AM peaks.

The negative quadrature lock is observed in figure 7.7. Bias set to $5.80 \mathrm{~mA}$ for the phase section, with front and back biased to $0 \mathrm{~mA}$. The gain is constant at $100 \mathrm{~mA}$, with SOA set to $98 \mathrm{~mA}$. Midrange photocurrent of $0.44-0.82 \mathrm{~mA}$ indicating operation near the linear portion of the quadrature slope. Quadrature operation is validated, as evident by the increased magnitude on the $S_{21}$ and photocurrent levels.

Point C, the trough of the MZI curve is presented in figure 7.8. This point typically contains reduced photocurrent values, as well as reduced amplitude modulation as well as frequency modulation responses. A reduction in the $S_{21}$ indicates reduced frequency modulation response present. Overall photocurrent range is reduced significantly to $0.02-0.56 \mathrm{~mA}$. This point, while not generally used in determining the response of the system, is necessary as a key point in 


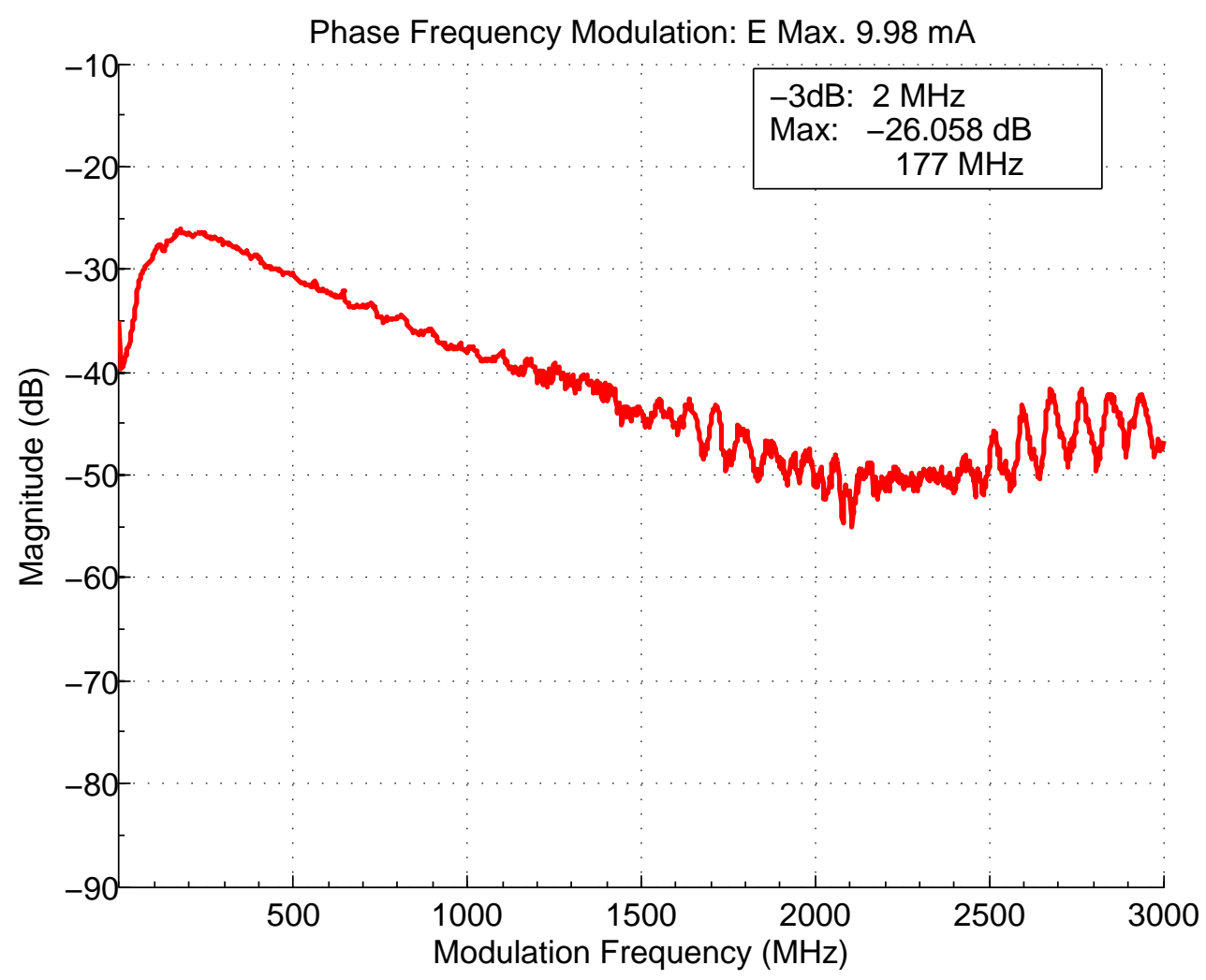

Figure 7.6: Phase Section FM Response at Maximum Photocurrent Intensity, represented by point $\mathrm{E}$ on figure 7.5. Primarily consistent of AM signal with maximum photocurrent, and minimal FM response. Bias conditions of $I_{P M}=9.98 \mathrm{~mA}, I_{B M}=I_{F M}=0 \mathrm{~mA}, I_{\text {Gain }}=100$ $\mathrm{mA}, I_{S O A}=98 \mathrm{~mA}$; Peak wavelength $\lambda=1318.540 \mathrm{~nm}$; Photocurrent range of $I_{P D}=[0.83-1.71 \mathrm{~mA}]$

determining and verifying the quadrature points.

Point D in figure 7.9 indicates the second quadrature point, however with a positive slope. Again, photocurrent observed is midrange from the peak and trough values. The AM signal is reduced, and the FM response is at a maximum observed by the $S_{21}$. The spacing between quadrature polarities is half the FSR of the interferometer. Given FSR of $100 \mathrm{pm}$, indicates each quadrature point is ideally 50 pm separated. Validation between successive quadrature regions can 
be verified is there exists $50 \mathrm{pm}$ different in wavelength between the response peaks. From the negative quadrature in figure 7.7 with $\lambda=1318.540$, to the newly proposed positive quadrature location with $\lambda=1318.580$ yields $\Delta \lambda \approx 40$ $\mathrm{pm}$. The deviance from the ideal $50 \mathrm{pm}$ is due to approximate quadrature locking from manual tuning, however is still predicted to be within the linear operating region.

Observing the $-3 \mathrm{~dB}$ bandwidth from the quadrature biased cases, and averaging between both polarities, indicates the effective frequency modulation limit $f_{\text {phase, } \max } \approx 250 \mathrm{MHz}$ for the phase section. An additional data set, presented in figure 7.10 performed with different bias currents yields an approximate modulation limit of $240 \mathrm{MHz}$, agreeing with the previously observed trial run.

The $\mathrm{RC}$ electrical parasitic limitations in chapter 5.4, with a similar phase section bias current of $5 \mathrm{~mA}$, yields a cutoff of $848 \mathrm{MHz}$. For the range of bias currents on the phase section, all practical bias conditions when frequency modulated have a significantly higher RC limitations frequency, and are likely relaxation limited. Therefore when frequency modulating the phase section, the experimentally determined limitations of $240 \mathrm{MHz}$ as an absolute maximum should be observed. 


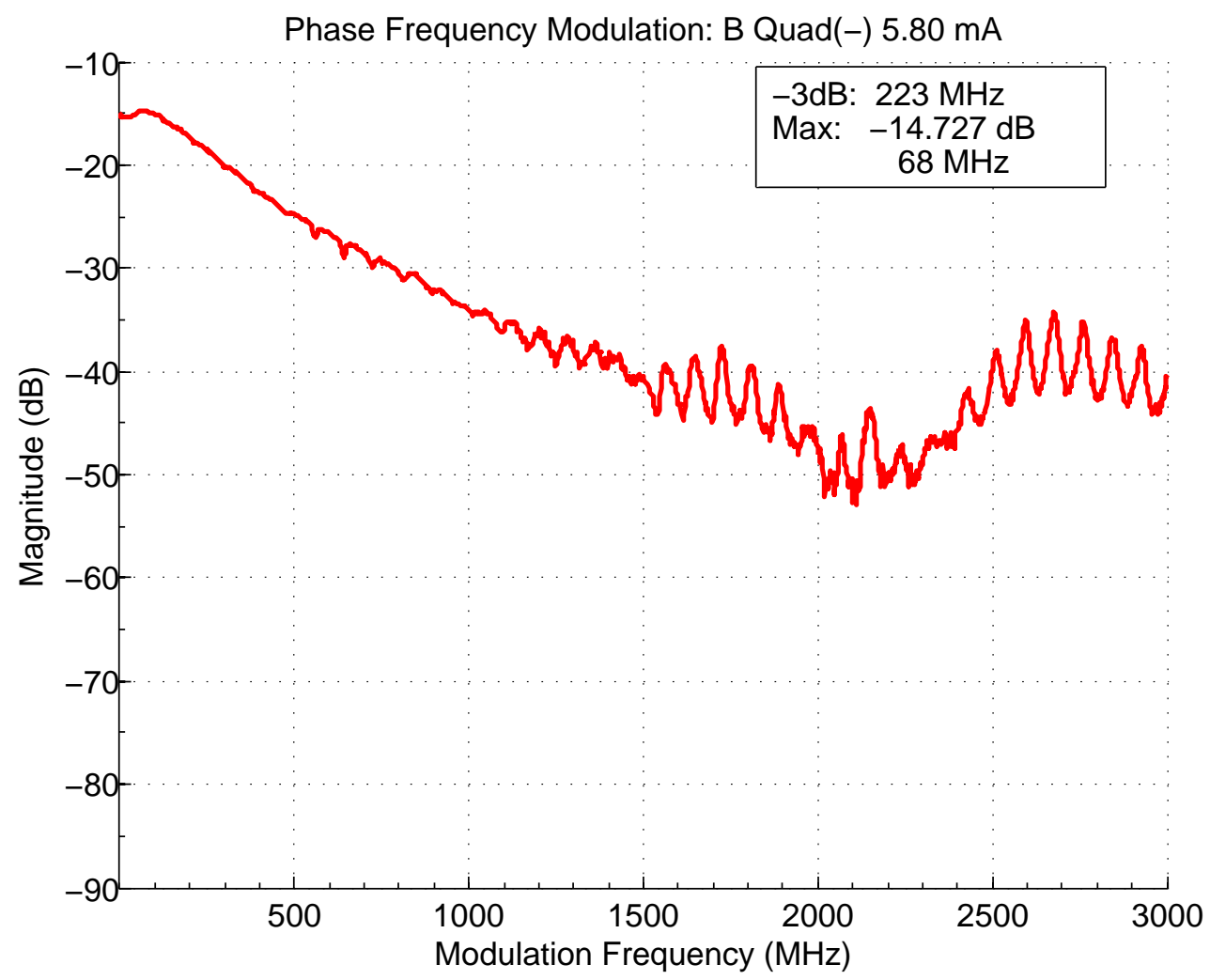

Figure 7.7: Phase Section FM Response at Quadrature(-), represented by point $\mathrm{E}$ on figure 7.5. Plot consists of reduced AM signal, midrange photocurrent, and maximal FM response observed. Bias conditions of $I_{P M}=5.80 \mathrm{~mA}, I_{B M}=I_{F M}=0 \mathrm{~mA}, I_{\text {Gain }}=100 \mathrm{~mA}, I_{S O A}=98 \mathrm{~mA}$; Peak wavelength $\lambda=1318.592 \mathrm{~nm}$; Photocurrent range of $I_{P D}=[0.44-$ $0.82 \mathrm{~mA}$ ]. Phase section maximum modulation is approximately 223 $\mathrm{MHz}$. 


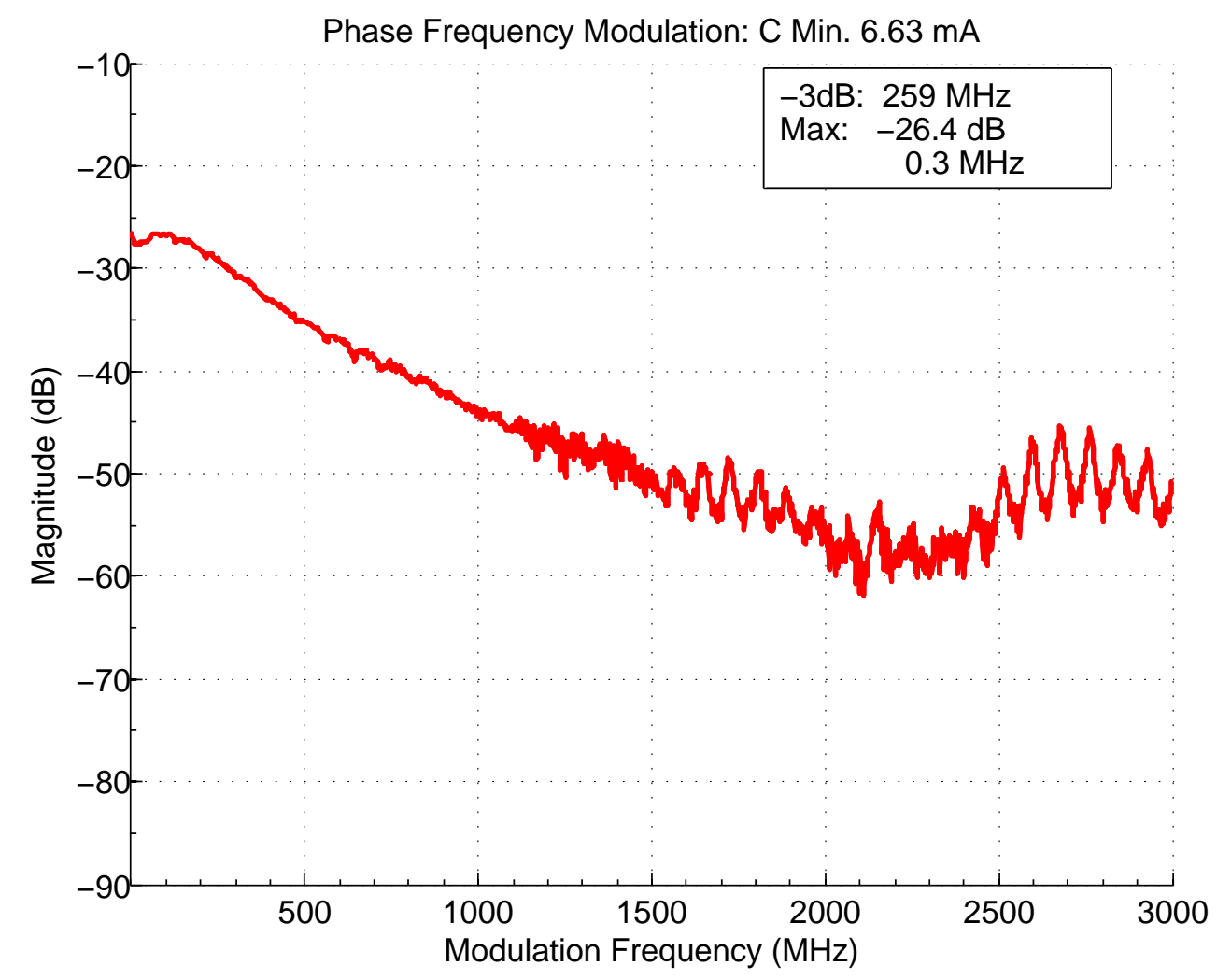

Figure 7.8: Phase Section FM Response at Minimum, represented by point $\mathrm{C}$ on figure 7.5. Plot consists of reduced AM signal, reduced photocurrent, and reduced FM response. Bias conditions of $I_{P M}=$ $6.63 \mathrm{~mA}, I_{B M}=I_{F M}=0 \mathrm{~mA}, I_{G a i n}=100 \mathrm{~mA}, I_{S O A}=98 \mathrm{~mA}$; Peak wavelength $\lambda=1318.580 \mathrm{~nm}$; Photocurrent range of $I_{P D}=[0.02-0.56$ $\mathbf{m A}]$ 


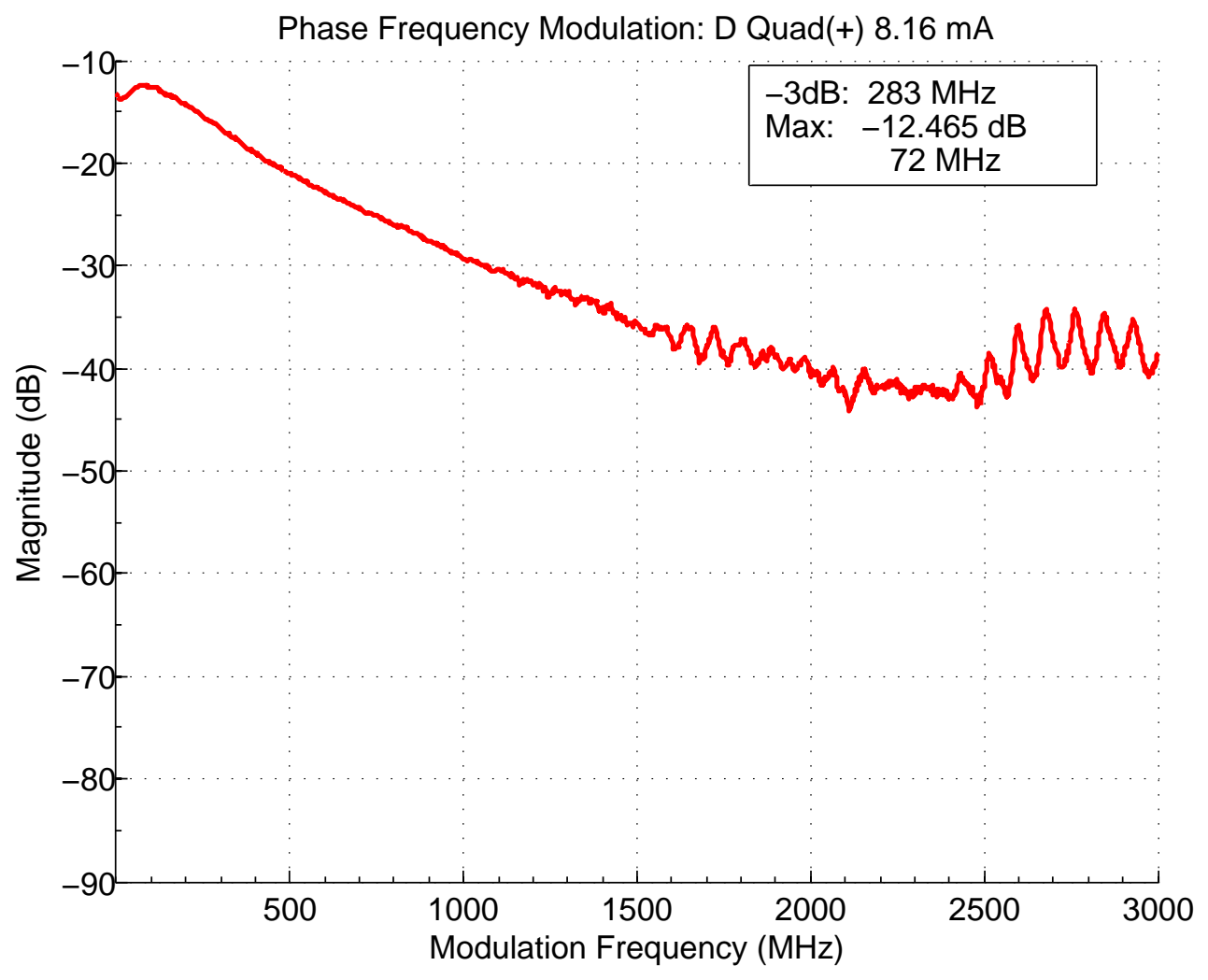

Figure 7.9: Phase Section FM Response at Quadrature $(+)$, represented by point D on figure 7.5. Plot consists of reduced AM signal, midrange photocurrent, and maximal FM response observed. Bias conditions of $I_{P M}=8.16 \mathrm{~mA}, I_{B M}=I_{F M}=0 \mathrm{~mA}, I_{\text {Gain }}=100 \mathrm{~mA}$, $I_{S O A}=98 \mathrm{~mA}$; Peak wavelength $\lambda=1318.562 \mathrm{~nm}$; Photocurrent range of $I_{P D}=[0.56-0.82 \mathrm{~mA}]$. Phase section maximum modulation is approximately $283 \mathrm{MHz}$. 




(a) Phase FM Point A: max. intensity,

$I_{P M}=4.00 \mathrm{~mA} ; \lambda=1318.610 \mathrm{~nm} ; I_{P D}$ $=[1.38-1.56 \mathrm{~mA}]$



(c) Phase FM Point C: min. intensity, $I_{P M}$

$=6.55 \mathrm{~mA} ; \lambda=1318.580 \mathrm{~nm} ; I_{P D}=[0.28-$

$0.62 \mathrm{~mA}]$

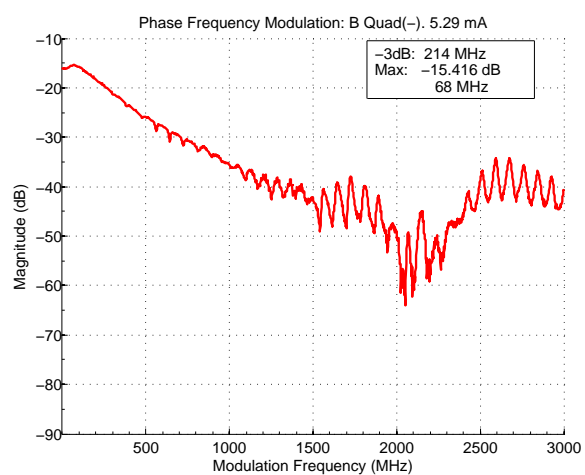

(b) Phase FM Point B: Quad(-), $I_{P M}=$ $5.29 \mathrm{~mA} ; \lambda=1318.592 \mathrm{~nm} ; I_{P D}=[0.83-$ $0.89 \mathrm{~mA}]$

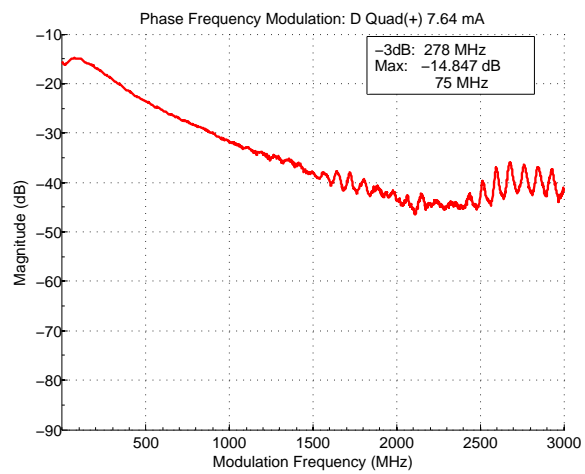

(d) Phase FM Point D: Quad(+), $I_{P M}=$ $7.64 \mathrm{~mA} ; \lambda=1318.558 \mathrm{~nm} ; I_{P D}=[0.88-$ $0.93 \mathrm{~mA}]$

Figure 7.10: Phase Section FM Response: Additional Data. $I_{P D}$ indicates photocurrent range, $\lambda$ indicates the peak wavelength observed, $I_{P M}$ is the phase bias current. Bias conditions of $I_{B M}=I_{F M}=0 \mathbf{~ m A}$, $I_{S O A}=98 \mathrm{~mA}$, and $I_{G A I N}=100 \mathrm{~mA}$ used for all tests; Quadrature bias is present in part (b) and (d). (a) represents maximum photocurrent case, while subfigure (c) is the minimal trough case of photocurrent. Results yield maximum modulation response on phase section of $f_{\text {phase } \text { max }} \approx 240 \mathrm{MHz}$. 


\subsection{FM Response on Front Mirror Section}

Following the theory and procedure from the phase modulation section, the front mirror is stimulated now. The same $17.5 \mathrm{GHz}(100 \mathrm{pm})$ interferometer is utilized for testing. The output power of the VNA, originally $0 \mathrm{dBm}$ for the phase tests, is lowered to $-10 \mathrm{dBm}$ to limit the frequency deviations within the FSR of the interferometer.

For testing, a stable SLM was determined via iterative tuning with bias currents of $I_{F M}=12.31 \mathrm{~mA}, I_{B M}=37.09 \mathrm{~mA}, I_{S O A}=98 \mathrm{~mA}, I_{G A I N}=100 \mathrm{~mA}$. The injection current for the phase was varied from $7.05-11.95 \mathrm{~mA}$ to achieve fine frequency tuning within the FSR of the interferometer. Figure 7.11, illustrates the four essential bias points on the MZI curve.

Point A is enlarged in figure 7.12. The phase current is biased at $7.05 \mathrm{~mA}$, with the same fixed front and back currents of 12.31 and $37.09 \mathrm{~mA}$ respectively. The gain bias is $100 \mathrm{~mA}$, with the SOA set to $98 \mathrm{~mA}$, a per the remainder of the modulation tests. Peak wavelength of $1316.901 \mathrm{~nm}$ with a photocurrent range of 0.93-1.05 mA indicates the top of the MZI curve with maximum amplitude and minimal FM response present.

Figure 7.13 indicates the negative quadrature bias for the front mirror section. The $S_{21}$ magnitude at maximum combined with midrange photocurrent indicate quadrature. The cutoff frequency, as measured from the $-3 \mathrm{~dB}$ point is approximately $190 \mathrm{MHz}$.

The minimum amplitude point is presented in figure 7.14. The photocurrent range is not nearly as distinct in terms of dynamic range for the front mirror as compared to the phase section. At it's lowest the front mirror photocurrent ranges from $0.57-0.71 \mathrm{~mA}$ (when the maximum in point A had $0.93-1.05 \mathrm{~mA}$ ), 

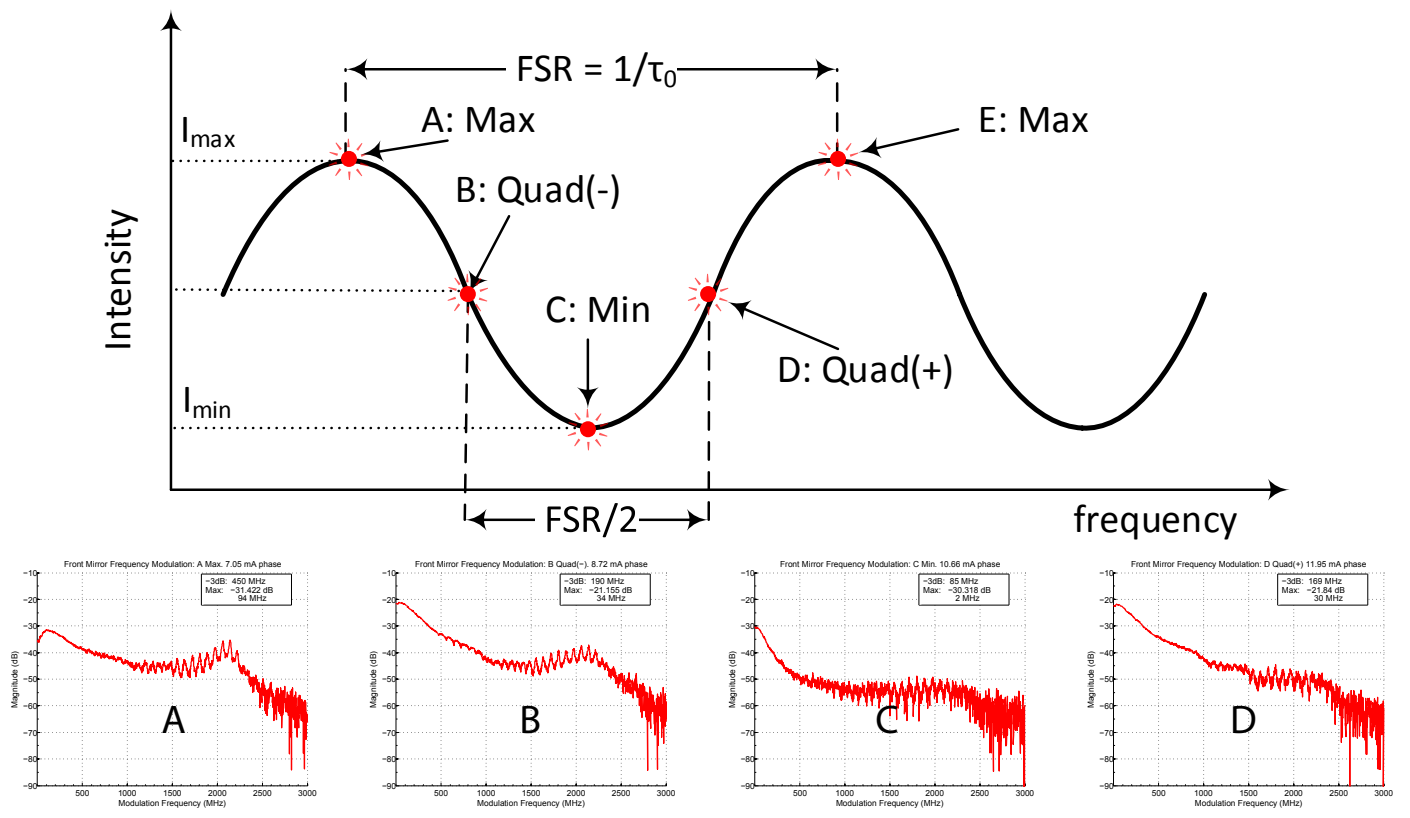

Figure 7.11: Front Mirror Frequency Modulation Data. Critical points on the interferometer output: (A) Maximum average amplitude; (B) Negative quadrature lock; (C) Minimum average amplitude; (D) Positive quadrature lock; $I_{F M}=12.31 \mathrm{~mA} ; I_{B M}=37.09 \mathrm{~mA} ; I_{S O A}=98$ $\mathrm{mA} ; I_{G A I N}=100 \mathrm{~mA}$; Varying phase current for tuning.

indicating greater amplitude influences from frequency modulation. FM response is also minimal at the point in the MZI curve.

The second quadrature point is presented in 7.15. All mirror bias currents remain constant from the test, with the phase current changed to $11.95 \mathrm{~mA}$. The cutoff frequency for the positive quadrature lock is $170 \mathrm{MHz}$.

Averaging the two cutoff frequencies from each quadrature point yields a maximum front mirror modulation $f_{\text {front, } \max } \approx 180 \mathrm{MHz}$. Comparing to the $\mathrm{RC}$ electrical parasitic limitations from before, with a similar front mirror bias current of $10 \mathrm{~mA}$, yields a cutoff of $1675 \mathrm{MHz}$. Even with small bias currents of 5 $\mathrm{mA}$, the $\mathrm{RC}$ limited frequency is $985 \mathrm{MHz}$. It is evident that for medium to large bias currents the parasitic limitations do not limit the modulation frequency. 


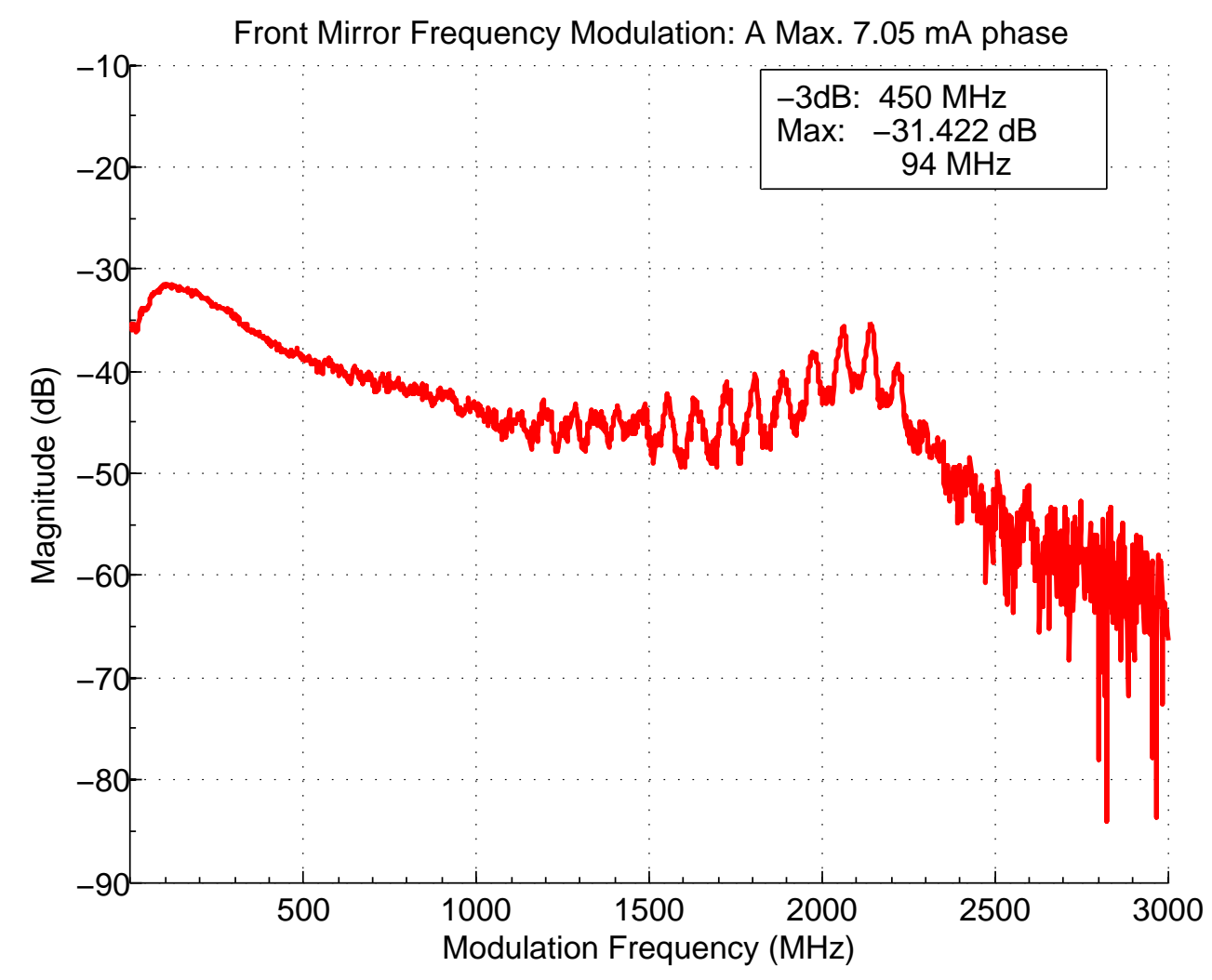

Figure 7.12: Front Mirror FM Response at Maximum, represented by point A on 7.11. Primarily consistent of AM signal with maximum photocurrent, and minimal FM response. Bias conditions of $I_{F M}=$ $12.31 \mathrm{~mA}, I_{B M}=37.09 \mathrm{~mA} ; I_{S O A}=98 \mathrm{~mA}, I_{G A I N}=100 \mathrm{~mA}$, and $I_{P M}$ $=7.05 \mathrm{~mA}$; Peak wavelength $\lambda=1316.901 \mathrm{~nm}$; Photocurrent range of $I_{P D}=[0.93-1.05 \mathrm{~mA}]$. 




Figure 7.13: Front Mirror FM Response at Quadrature(-), represented by point $\mathrm{B}$ on 7.11. Reduced AM signal, midrange photocurrent, and maximal FM response. Bias conditions of $I_{F M}=12.31 \mathrm{~mA}, I_{B M}=$ $37.09 \mathrm{~mA} ; I_{S O A}=98 \mathrm{~mA}, I_{G A I N}=100 \mathrm{~mA}$, and $I_{P M}=8.72 \mathrm{~mA}$; Peak wavelength $\lambda=1316.885 \mathrm{~nm}$; Photocurrent range of $I_{P D}=[0.89-0.91$ $\mathrm{mA}$ ]. Front mirror maximum modulation is approximately $190 \mathrm{MHz}$. 


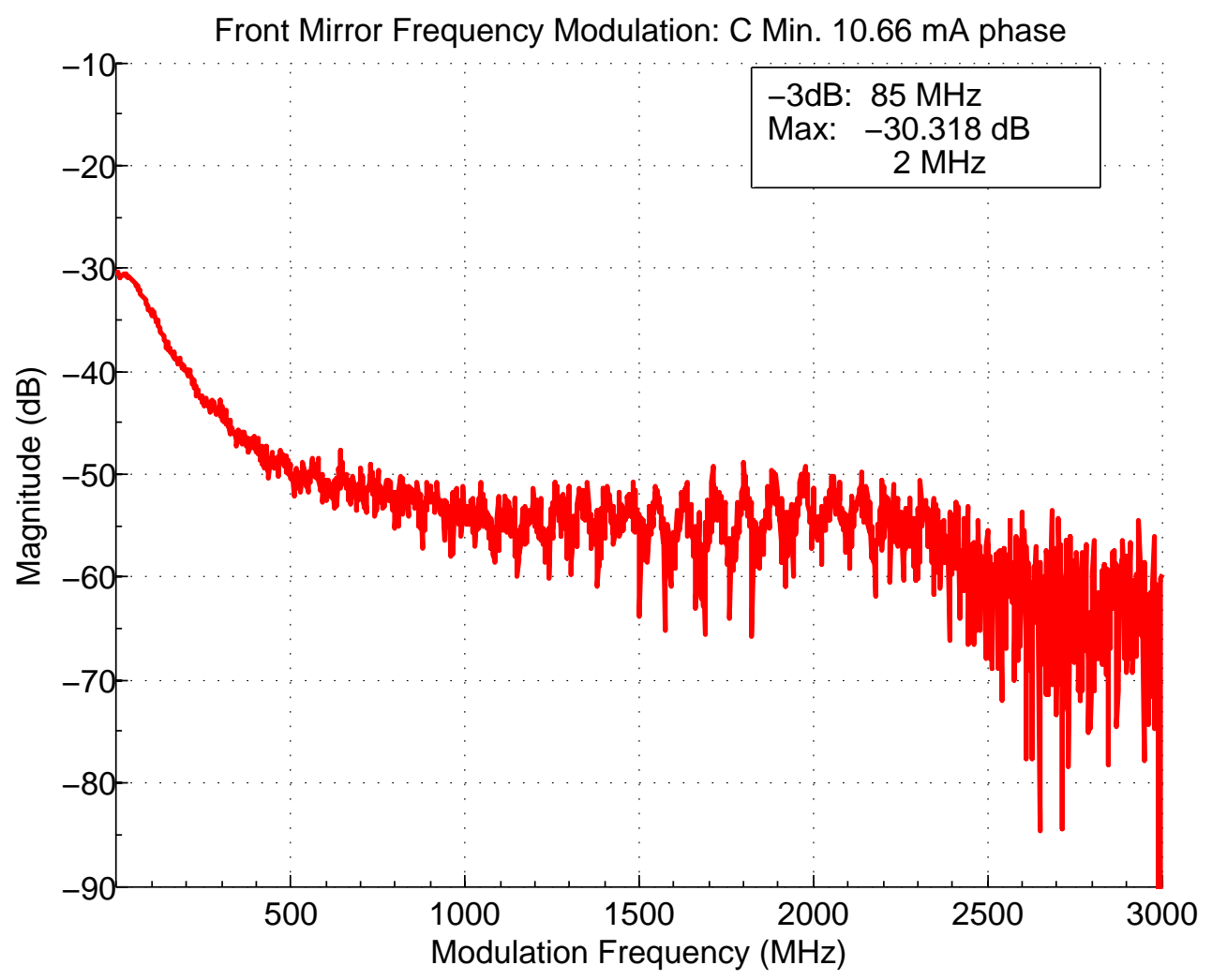

Figure 7.14: Front Mirror FM Response, represented by point $\mathrm{C}$ on 7.11. Reduced AM signal, reduced photocurrent, reduced FM response. Bias conditions of $I_{F M}=12.31 \mathrm{~mA}, I_{B M}=37.09 \mathrm{~mA} ; I_{S O A}$ $=98 \mathrm{~mA}, I_{G A I N}=100 \mathrm{~mA}$, and $I_{P M}=10.66 \mathrm{~mA}$; Peak wavelength $\lambda$ $=1316.861 \mathrm{~nm} ;$ Photocurrent range of $I_{P D}=[0.57-0.71 \mathrm{~mA}]$ 


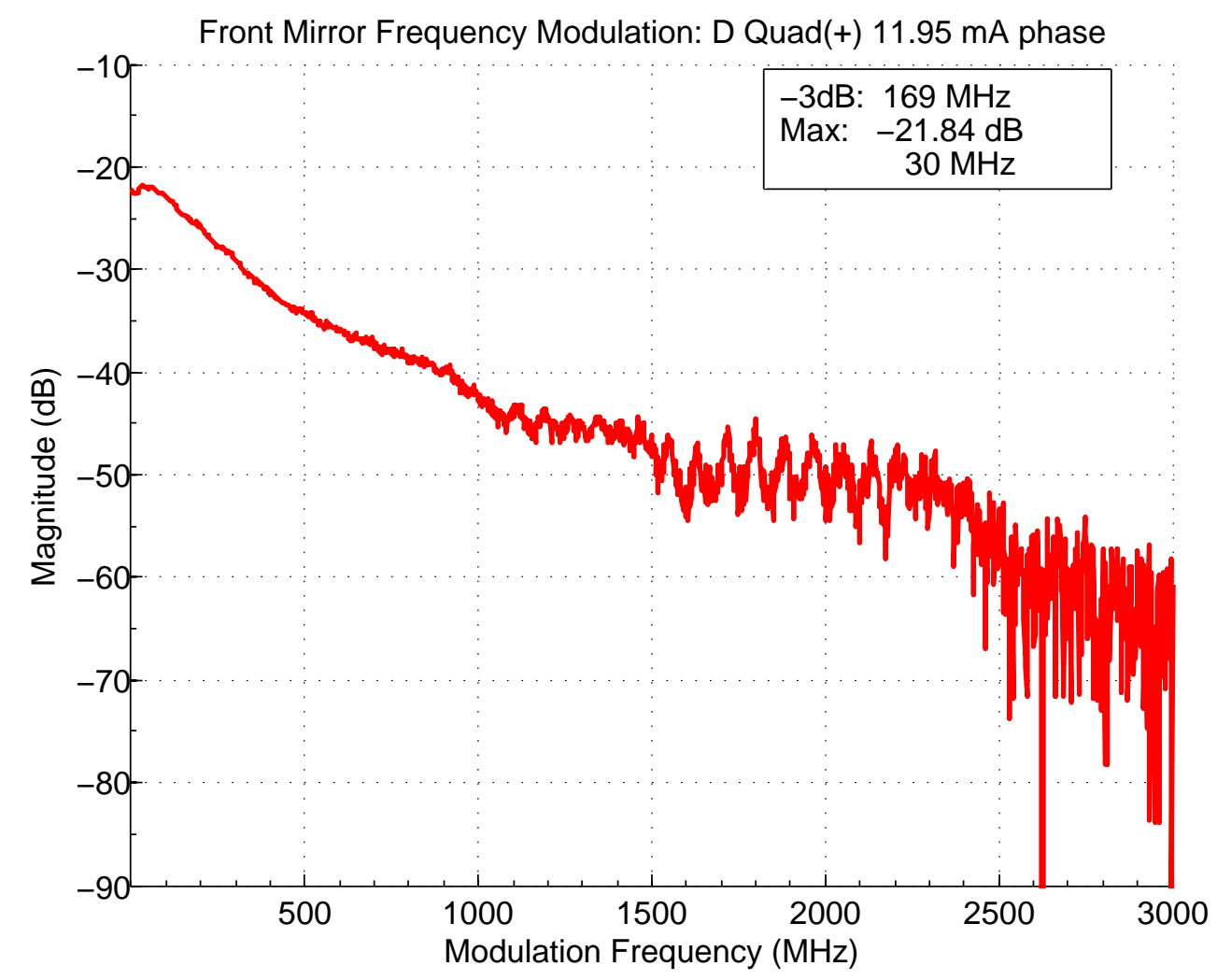

Figure 7.15: Front Mirror FM Response at Quadrature(+), represented by point $\mathrm{D}$ on 7.11. Reduced AM signal, midrange photocurrent, and maximal FM response. Bias conditions of $I_{F M}=12.31 \mathrm{~mA}$, $I_{B M}=37.09 \mathrm{~mA} ; I_{S O A}=98 \mathrm{~mA}, I_{G A I N}=100 \mathrm{~mA}$, and $I_{P M}=11.95$ $\mathrm{mA}$; Peak wavelength $\lambda=1316.847 \mathrm{~nm}$; Photocurrent range of $I_{P D}=$ [0.68-0.76 mA]. Front mirror maximum modulation is approximately $170 \mathrm{MHz}$ 


\subsection{FM Response on Back Mirror Section}

The final portion of the frequency control sections is performed on the back mirror. For these tests, as per the phase and front sections only the Back Mirror is excited with frequency sweeps. Through iterative tuning, fixed bias points of $I_{F M}=17.91 \mathrm{~mA}, I_{B M}=27.31 \mathrm{~mA}, I_{S O A}=98 \mathrm{~mA}$, and $I_{G A I N}=100 \mathrm{~mA}$ were obtained. For all of the cases the phase section biased was used to tune the frequency throughout the FSR of the interferometer. The VNA power level is set to $-10 \mathrm{dBm}$, as for the front mirror case. Figure 7.16 is a summary of the major key points on the MZI curve, presented in the same manner as for previous sections.



Figure 7.16: Back Mirror Frequency Modulation Data. Critical points on the interferometer output: (A) Maximum average amplitude; (B) Negative quadrature lock; (C) Minimum average amplitude; (D) Positive quadrature lock; $I_{F M}=17.91 \mathrm{~mA} ; I_{B M}=27.31 \mathrm{~mA} ; I_{S O A}=98$ $\mathrm{mA} ; I_{G A I N}=100 \mathrm{~mA}$; Varying phase current for tuning.

Figure 7.17 represents the maximum amplitude point A on the graph. Recall 
that the maximum amplitude is on the peak of the MZI curve, indicating high photocurrent values, high AM response, and minimal FM response as indicated by the reduced $S_{21}$ magnitude.



Figure 7.17: Back Mirror FM Response at Maximum Intensity, represented by point $\mathrm{A}$ on 7.16. Primarily consistent of AM signal with maximum photocurrent, and minimal FM. Bias conditions of $I_{F M}=$ $17.91 \mathrm{~mA}, I_{B M}=27.31 \mathrm{~mA}, I_{S O A}=98 \mathrm{~mA}, I_{G A I N}=100 \mathrm{~mA}$, and $I_{P M}$ $=8.70 \mathrm{~mA}$; Peak wavelength $\lambda=1320.769 \mathrm{~nm}$; Photocurrent range of $I_{P D}=[0.96-1.01 \mathrm{~mA}]$

Figure 7.18 is the first quadrature negative slope on point B. This point exhibits high FM response, with reduced AM response, and midrange photocurrent values. The $-3 \mathrm{~dB}$ cutoff is determined to be approximately $124 \mathrm{MHz}$.

The minimum intensity point $\mathrm{C}$ is represented in figure 7.19. Again, reduced 


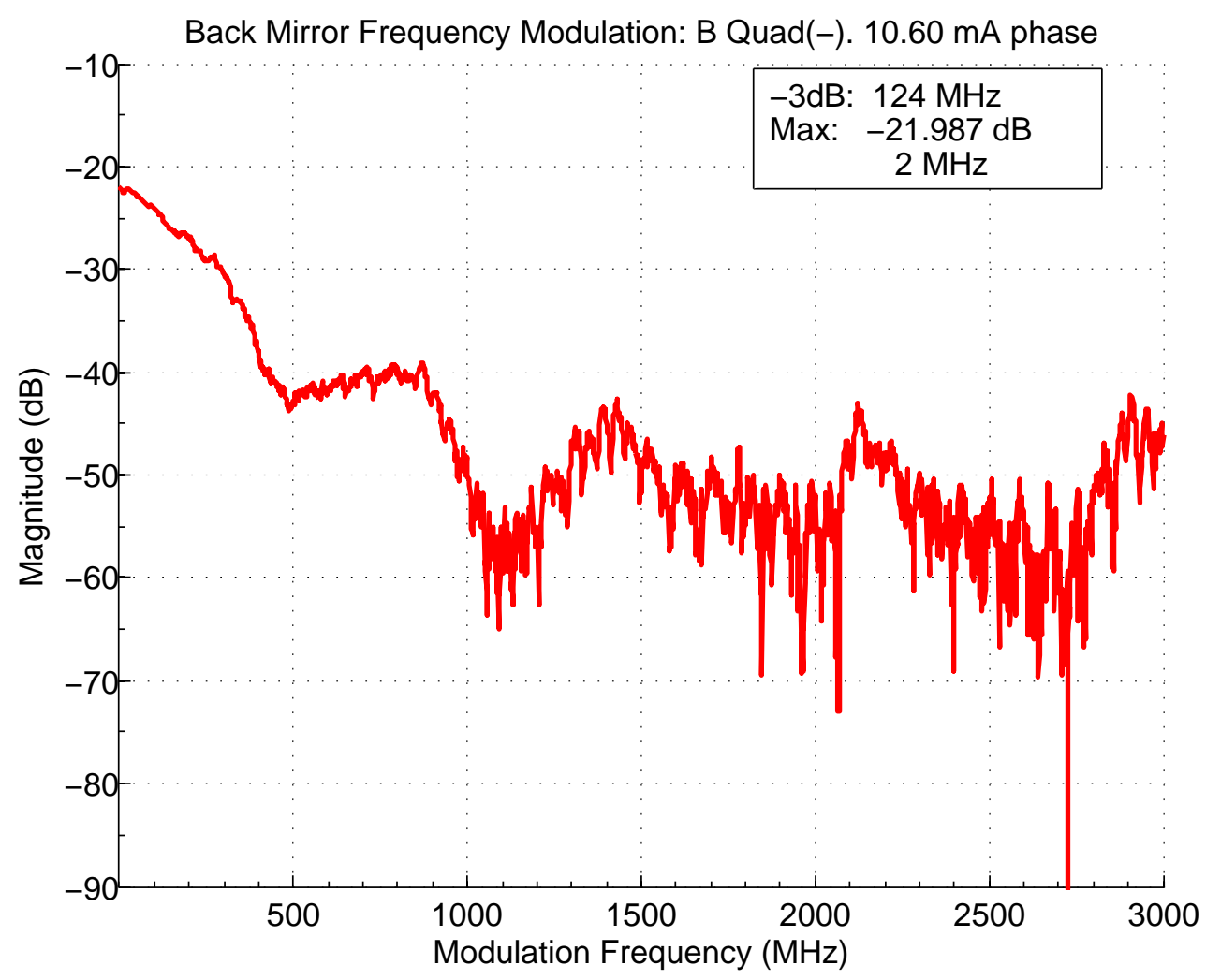

Figure 7.18: Back Mirror FM Response at Quadrature(-), represented by point $\mathrm{B}$ on 7.16. Reduced AM signal, midrange photocurrent, and maximal FM response. Bias conditions of $I_{F M}=17.91 \mathrm{~mA}, I_{B M}=$ $27.31 \mathrm{~mA}, I_{S O A}=98 \mathrm{~mA}, I_{G A I N}=100 \mathrm{~mA}$, and $I_{P M}=10.60 \mathrm{~mA}$; Peak wavelength $\lambda=1320.753 \mathrm{~nm}$; Photocurrent range of $I_{P D}=[0.70-0.75$ $\mathrm{mA}$ ]. Back mirror maximum modulation is approximately $124 \mathrm{MHz}$.

photocurrent, AM and FM response are found in the trough region of the MZI curve.

Finally figure 7.20 is the last quadrature point captured within the FSR range. The modulation frequency was measured to be approximately $98 \mathrm{MHz}$.

Taking the both polarities of quadrature bias, and their experimentally determined cutoff frequencies yields and average of $f_{\text {back,max }} \approx 110 \mathrm{MHz}$. This indicates the approximate maximum modulation frequency at which the back mirror can 




Figure 7.19: Back Mirror FM Response at Minimum Intensity, represented by point $\mathrm{C}$ on 7.16. Reduced $\mathrm{AM}$ signal, reduced photocurrent, reduced FM response. Bias conditions of $I_{F M}=17.91 \mathrm{~mA}, I_{B M}=27.31$ $\mathrm{mA}, I_{S O A}=98 \mathrm{~mA}, I_{G A I N}=100 \mathrm{~mA}$, and $I_{P M}=12.06 \mathrm{~mA}$; Peak wavelength $\lambda=1320.741 \mathrm{~nm}$; Photocurrent range of $I_{P D}=[0.39-0.53 \mathrm{~mA}]$

be changed. The back mirror bias currents of approximately $27 \mathrm{~mA}$ in these tests are compared to the electrical RC parasitics from chapter 5.3. For the case of 20 $\mathrm{mA}$, the electrical estimate for the cutoff frequency was $2037 \mathrm{MHz}$. Increasing biasing current only improves the RC cutoff estimate. Even for the lowest bias of $5 \mathrm{~mA}$ for the front mirror, the $\mathrm{RC}$ limit is $585 \mathrm{MHz}$ - several times greater than experimentally determined frequency modulation cutoff measurements. 


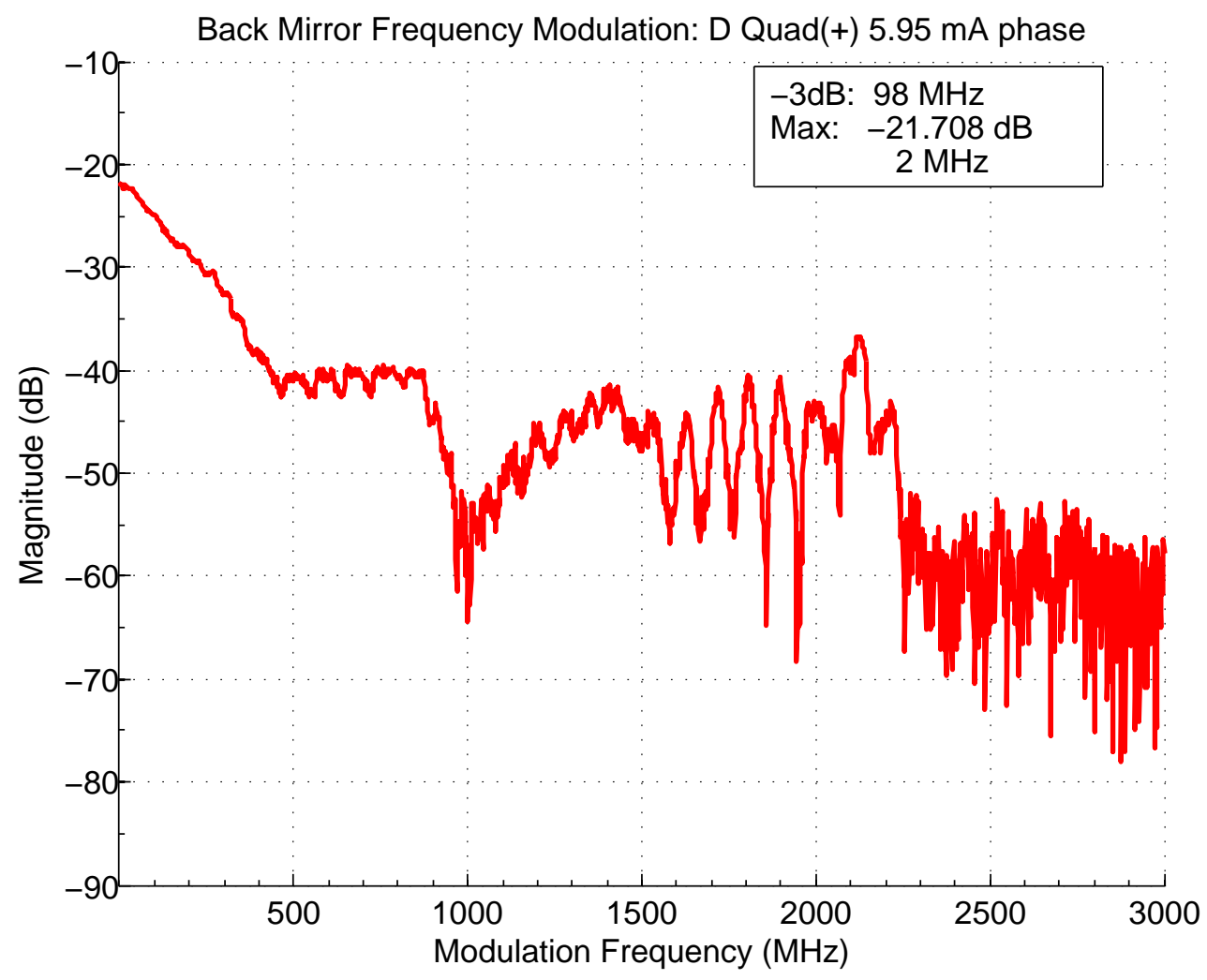

Figure 7.20: Back Mirror FM Response at Quadrature(+), represented by point $\mathrm{D}$ on 7.16. Reduced $\mathrm{AM}$ signal, midrange photocurrent, and maximal FM response. Bias conditions of $I_{F M}=17.91 \mathrm{~mA}, I_{B M}=$ $27.31 \mathrm{~mA}, I_{S O A}=98 \mathrm{~mA}, I_{G A I N}=100 \mathrm{~mA}$, and $I_{P M}=5.95 \mathrm{~mA}$; Peak wavelength $\lambda=1320.797 \mathrm{~nm}$; Photocurrent range of $I_{P D}=[0.59-0.66$ $\mathrm{mA}$ ]. Back mirror maximum modulation is approximately $98 \mathrm{MHz}$. 


\subsection{Summary of FM Responses}

The frequency modulation response measurements of the Phase, Front Mirror, and Back Mirror sections provide details useful for determining how fast the frequency can be tuned on the laser. From chapter 5 detailing the equivalent circuit models, this yielded approximate limits based on the RC parasitics of each port. However, the parasitics are not the only element in play which limits the maximum modulation rate. In this chapter frequency modulation tests were performed on each of the tuning sections to determine the maximum rates. Table 7.2 summaries results from the RC parasitic limited data from before, and the frequency modulation limited bandwidths from this chapter. For the case of the FM tests, only discrete bias currents were chosen, therefore for comparison purposes all of the RC parasitic bias cases are shown. It is evident that even for the smallest of bias currents, the maximum modulation for the tuning elements is limited by phenomenon in the laser, likely due to relaxation resonance, and is not the RC limited.

Table 7.2: RC Parasitic and FM Response Limited Bandwidth Comparison. Each section of the laser presents the bias currents for testing from the RC parasitics (chapter 5), and the corresponding bandwidths. The Frequency Modulation (FM) tests from this chapter, and the bias current under test, are compared. For all cases it is evident that the modulation bandwidth is limited by relaxation oscillations and not the RC parasitic limited bandwidth.

\begin{tabular}{|c||ccccc|c|c|}
\hline Section & \multicolumn{5}{|c|}{ RC Limited BW and Bias } & FM Limited BW and Bias & Units \\
\hline \hline \multirow{2}{*}{ Phase } & 5 & 7.5 & 10 & 15 & 20 & $5.8,8.2$ & $(\mathrm{~mA})$ \\
\cline { 2 - 9 } & 848 & 1072 & 1213 & 1536 & 1816 & 240 & $(\mathrm{MHz})$ \\
\hline \hline \multirow{2}{*}{ Front } & 5 & 10 & 20 & 30 & 35 & 12.3 & $(\mathrm{~mA})$ \\
\cline { 2 - 9 } & 985 & 1675 & 2195 & 2697 & 2920 & 180 & $(\mathrm{MHz})$ \\
\hline \hline \multirow{2}{*}{ Back } & 5 & 10 & 20 & 40 & 60 & 27.3 & $(\mathrm{~mA})$ \\
\cline { 2 - 8 } & 585 & 1565 & 2037 & 2324 & 2521 & 110 & $(\mathrm{MHz})$ \\
\hline
\end{tabular}




\section{Chapter 8}

\section{Linewidth}

The final performance metric under study in this report is the characterization of laser's spectral width. One of the key advantages of a laser optical output besides being highly monochromatic is the extremely narrow spectral width, known as the linewidth. In general, a narrower linewidth indicates a tighter spectral width and higher performance in high speed communications or imaging. Linewidth is defined as the full-width half-maximum (FWHM) of the laser's power spectrum.

\subsection{Test Setup}

Recall that stimulated emission creates two waves in phase from one initial wave. The temporal coherence refers to the phase relationship as a function of time [30]. A perfectly temporally coherent beam would vary phase uniformly with time. Linewidth is heavily related to the coherence time or coherence length. Typical causes of linewidth broadening include $1 / f$ noise [31], shot noise in the mirrors, and spontaneous emission in the cavity [25]. The coherence time for a 
Lorentzian profile is

$$
\tau_{c}=\frac{1}{\pi \Delta \nu}
$$

where $\Delta \nu$ is the linewidth. Clearly from the inverse relationship of equation 8.1, as linewidth decreases the coherence time increases, indicating a tighter spectral linewidth. In addition to the coherence time, another term known as the coherence length, $L_{c}$ is useful. Relating it to coherence time yields

$$
L_{c}=\nu_{g} \tau_{c}
$$

with the group velocity of light $\nu_{g}=c / n_{g}$, and $n_{g}$ is the group velocity index, also known as the refractive index. For standard optical fiber $n_{g} \approx 1.47$.

A typical OSA is unable to perform such a measurement due to the relatively limited resolution [25]. Figure 7.1 indicates the narrow width of the linewidth, on the order of $\sim 10$ 's MHz. Even very small wavelength changes on the order of fractions of nanometers results in frequency changes of several gigahertz, beyond the limit of an OSA. An alternate measurement approach for measuring several megahertz range linewidths is required.

An interferometer, similar to the one used previously for frequency conversion is utilized to perform the linewidth measurements. The HP 11980A fiber optic interferometer contains a large unbalanced length of fiber, inducing a time delay, $\tau_{0}$, of approximately $3.5 \mu \mathrm{s}$. Both paths are recombined with an additional coupler, of which the output is sent to a photodetector. A key difference between this interferometer is the addition of a polarization controller (PC) to modify the polarization state for maximum signal integrity, as the interferometer does 
not preserve the initial polarization. The complete experimental testing arrangement is detailed in figure 8.1. The Agilent Lightwave Switch is used to physically switch the fiber optic path from the photodetector to the OSA for viewing. A partial power splitter would create undesired reflections, potentially corrupting the linewidth measurements. Therefore the electrically controlled fiber switcher physically reroutes the fiber optic inside with minimal measured insertion loss $<1 \mathrm{~dB}$.



Figure 8.1: Delayed Self-Homodyne Linewidth Test Setup. DC electrical lines are denoted with green arrows, SMA based coaxial connections with gray arrows, and fiber optical links with blue arrows.

While there are several interferometric methods to measure the linewidth, the delayed self-homodyne approach allows reduced complexity in required external modulators (frequency shifters for the heterodyne case), while still providing very high resolution measurements. The isolator is necessary to prevent backscatter from traveling back into the laser, artificially increasing or decreasing the measured linewidth depending on the phase of the feedback [32]. The differential time delay is approximately $3.5 \mu s$ between the two unbalanced fiber legs. If the 
coherence time of the laser, $\tau_{C}$ is less than the differential delay $\tau_{0}$ of the interferometer, when the two signals recombine they will be uncorrelated. The net effect of two uncorrelated signals mixing together can be thought of as two separate lasers, each with identical linewidths and center frequencies $\nu_{0}$ [32]. It is necessary for the delayed self-homodyne to operate only with uncorrelated signals.

The two mixed optical signals produce current on the photodetector consisting of the autocorrelation of the electric field spectrum $S_{E}(f)$ [32]. Figure 8.2 graphically details the electric field of two signals, each produced from a different arm of the interferometer and centered at $\nu_{0}$. It is important to note that frequencies denoted with $\nu$ typically represent optical frequencies, which occur at $229 \mathrm{THz}$ for $1310 \mathrm{~nm}$. When measuring electrical frequencies (i.e. below several gigahertz), the standard $f$ is used to represent frequency. The final output spectrum displayed on an Electrical Spectrum Analyser (ESA) from the photodetector is centered around $0 \mathrm{~Hz}$. The linewidth is simply found on the ESA at the $-3 \mathrm{~dB}$ points. Although this only measures half of the width resulting from the autocorrelation, the autocorrelation process doubled the original linewidth by a factor of two for Lorentzian lineshapes, therefore measuring the positive folded side results in the correct $\Delta \nu$.

\subsection{Results}

The characterization of linewidth is not a static value, can can vary substantially with bias current and other external influences. A two-dimensional course matrix for the front and back mirror is presented in figure 8.3. The back mirror is adjust from $0.00-6.46 \mathrm{~mA}$, while the front mirror is adjusted from $0.00-4.44$ $\mathrm{mA}$. For each of these tests, bias conditions of $I_{P M}=0 \mathrm{~mA}, I_{S O A}=98 \mathrm{~mA}$, 


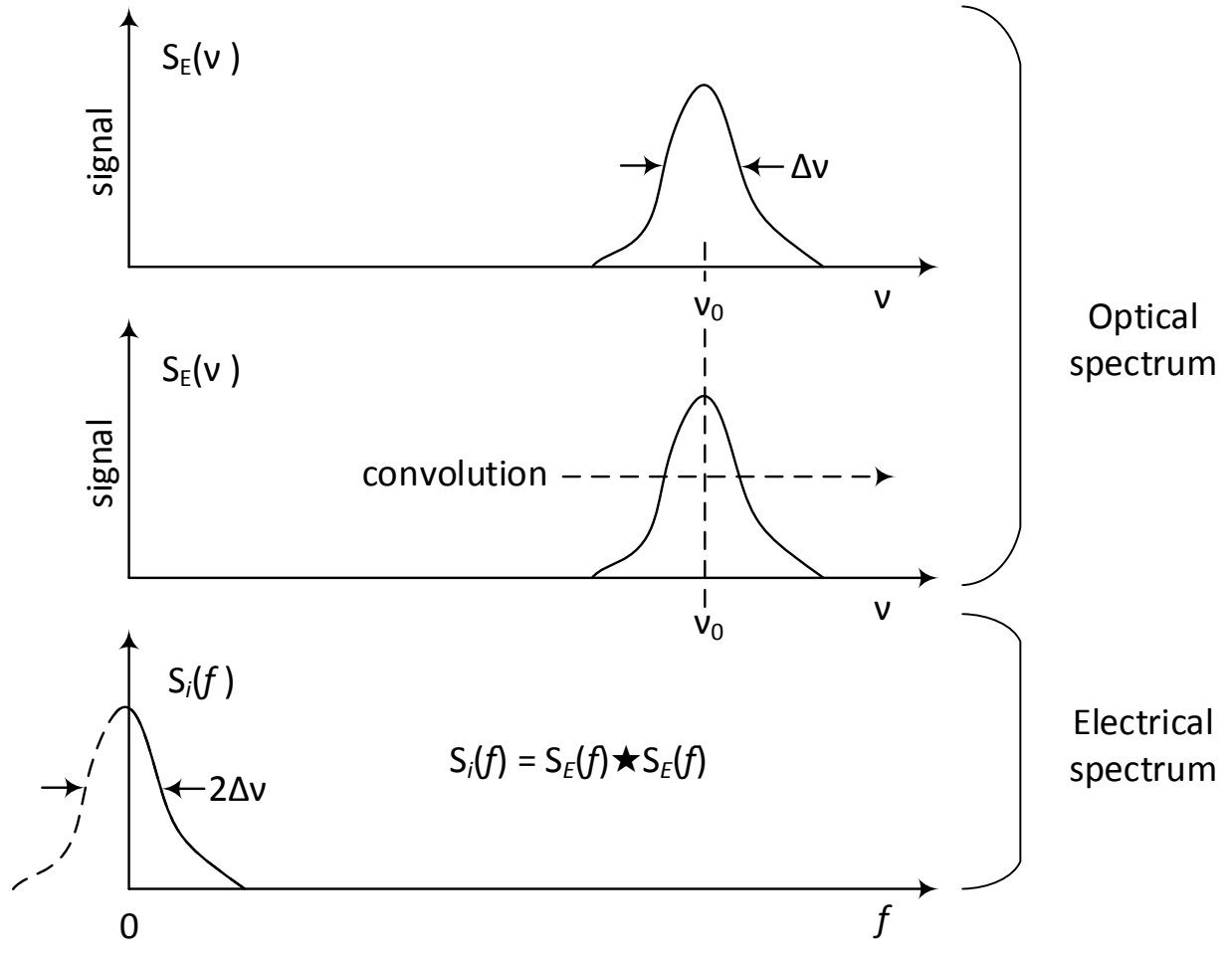

Figure 8.2: Delayed Self-Homodyne Linewidth Autocorrelation. Two optical signal centered at $\nu_{0}$ are recombined at the coupler. Through the autocorrelation function, the output is twice the spectral width and is centered at $0 \mathrm{~Hz}$. Therefore the linewidth, $\Delta \nu$, is measuring the $-3 \mathrm{~dB}$ point of the signal.

and $I_{G A I N}=100 \mathrm{~mA}$ are held constant. According the the mapping profile, the further away from the origin bias point the worse the linewidth. Especially in the case of the front mirror when back mirror is fixed bias at $0 \mathrm{~mA}$, variations the front mirror current greatly affect linewidth performance compared to only adjusting back mirror.

The course manual mapping profile shows that for operating near the origin the highest performance linewidth is observed. Finer resolution for each section independently is investigated in figure 8.4 for the back mirror variations and figure 8.5 for only front mirror. 


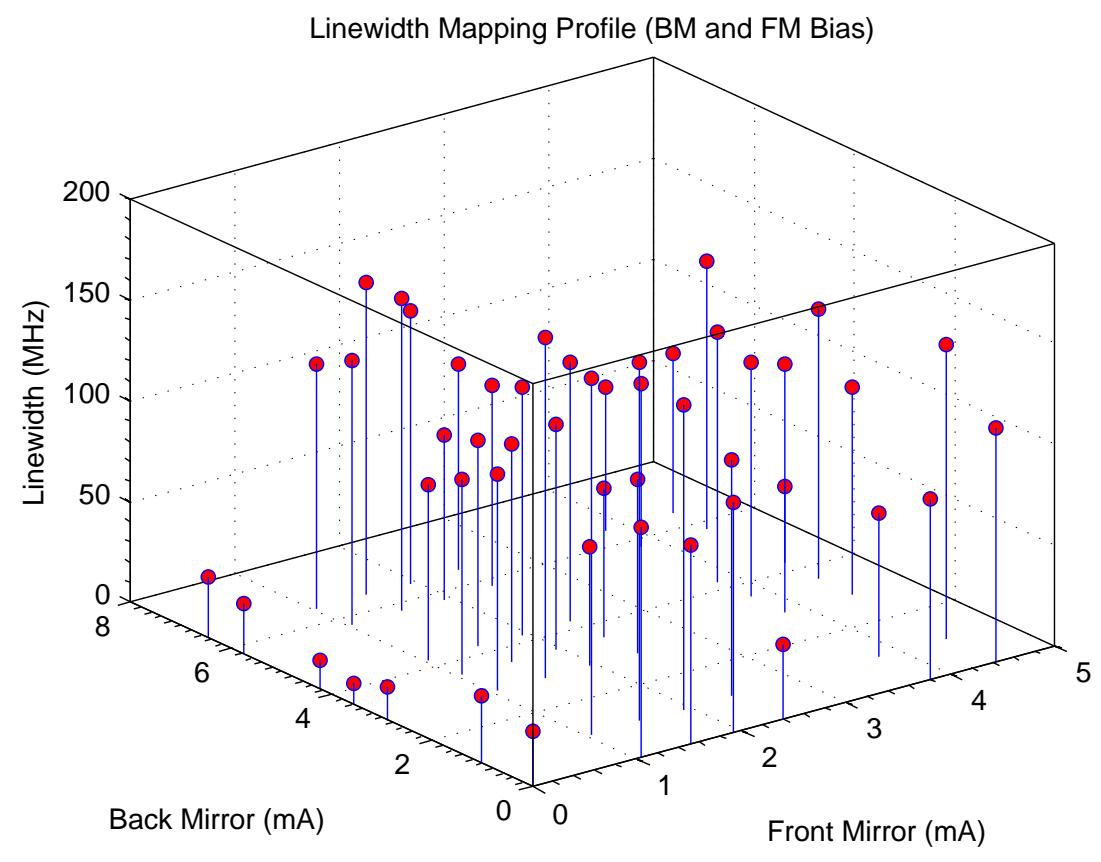

Figure 8.3: Linewidth Comparison Matrix for variations on front and back mirror current. Front and Back are varied, with $I_{P M}=\mathbf{0} \mathbf{m A}, I_{S O A}$ $=98 \mathrm{~mA}$, and $I_{G A I N}=100 \mathrm{~mA}$. Best linewidth performance is located at the origin of zero bias for front and back mirrors. Further increasing bias current in each mirror leads to an increase in the linewidth. A maximum of approximately $200 \mathrm{MHz}$ linewidth is noted in the testing range of currents of $0-7 \mathrm{~mA}$ for the back, and $0-5 \mathrm{~mA}$ for the front mirrors. 




Figure 8.4: Linewidth for Back Mirror Variations Only. Back Mirror varied, with $I_{F M}=0 \mathrm{~mA}, I_{P M}=0 \mathrm{~mA}, I_{S O A}=98 \mathrm{~mA}$, and $I_{G A I N}=100$ $\mathrm{mA}$. Linewidth generally less than $50 \mathrm{MHz}$ for most lower currents, inreasing up to approximately $300 \mathrm{MHz}$ from 16-20 mA. 


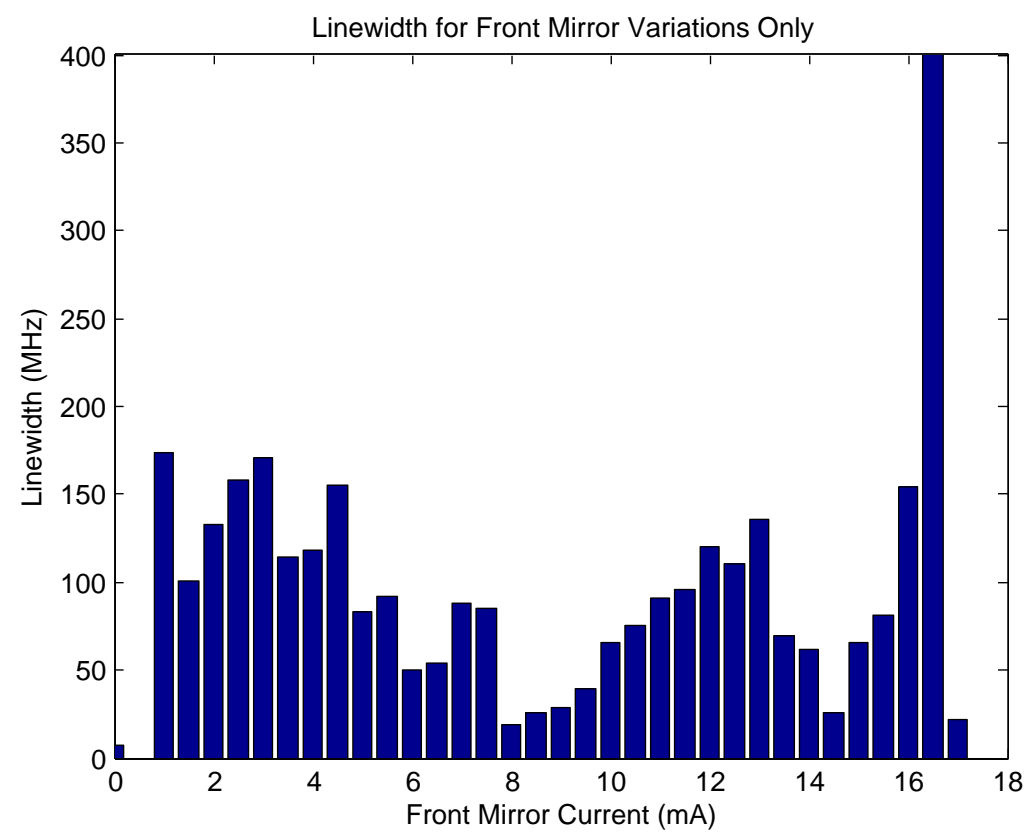

Figure 8.5: Linewidth for Front Mirror Variations Only. Front Mirror varied, with $I_{B M}=0 \mathrm{~mA}, I_{P M}=0 \mathrm{~mA}, I_{S O A}=98 \mathrm{~mA}$, and $I_{G A I N}=$ $100 \mathrm{~mA}$. For low to moderate bias currents the linewidth is relatively stable at less than $150 \mathrm{MHz}$. 
The same trend is visible in both mirror sections of increased bias current worsening linewidth. The several peaks and valleys in the individual mirror linewidths plots is likely due to traversing around the mode pads of the laser. Linewidth will be best when directly in the center of the mode pad.

An modification on the delayed self-homodyne linewidth measurement was performed to obtain a full two sided response. Referring back to the test setup in figure 8.1, notice the optional RF Synthesizer and biasing tee present. By modulating the gain section of the laser with a high frequency carrier stimulus the linewidth is now convolved around the modulation frequency $f_{\text {mod }}$, and is present at each harmonic, shown in figure 8.6 [33].

The linewidth now appears at the $0 \mathrm{~Hz}$ origin as before, but also centered as a two sided $(2 \Delta \nu)$ spectrum at each harmonic. For this test bias conditions of $I_{F M}=0 \mathrm{~mA}, I_{B M}=0 \mathrm{~mA}, I_{P M}=0 \mathrm{~mA}, I_{S O A}=98 \mathrm{~mA}$, and $I_{G A I N}=$ $100 \mathrm{~mA}$ were used. A modulation stimulus of $450 \mathrm{MHz}$ with $-4 \mathrm{dBm}$ power is sent into the Gain section of the laser. This approach is known as measuring the modulation sidebands in a delayed self-homodyne experiment. Measuring the linewidth traditional one sided measurement yields $23.0 \mathrm{MHz}$, where the linewidth derived from the sideband measurement produces $24.2 \mathrm{MHz}$.

The traditional delayed self homodyne test, with identical bias conditions of $I_{F M}=0 \mathrm{~mA}$ and $I_{B M}=0 \mathrm{~mA}$, yielded a linewidth of $26.3 \mathrm{MHz}$. This agrees closely to the $24.2 \mathrm{MHz}$ for the double-sided test. Either method is appropriate for measuring the linewidth, providing accurate results for SG-DBR linewidth measurements down to several hundred kilohertz [32]. The single-sided measurement method may be preferred, as it does not require the external RF synthesizer for testing compared to the double-sided test. 


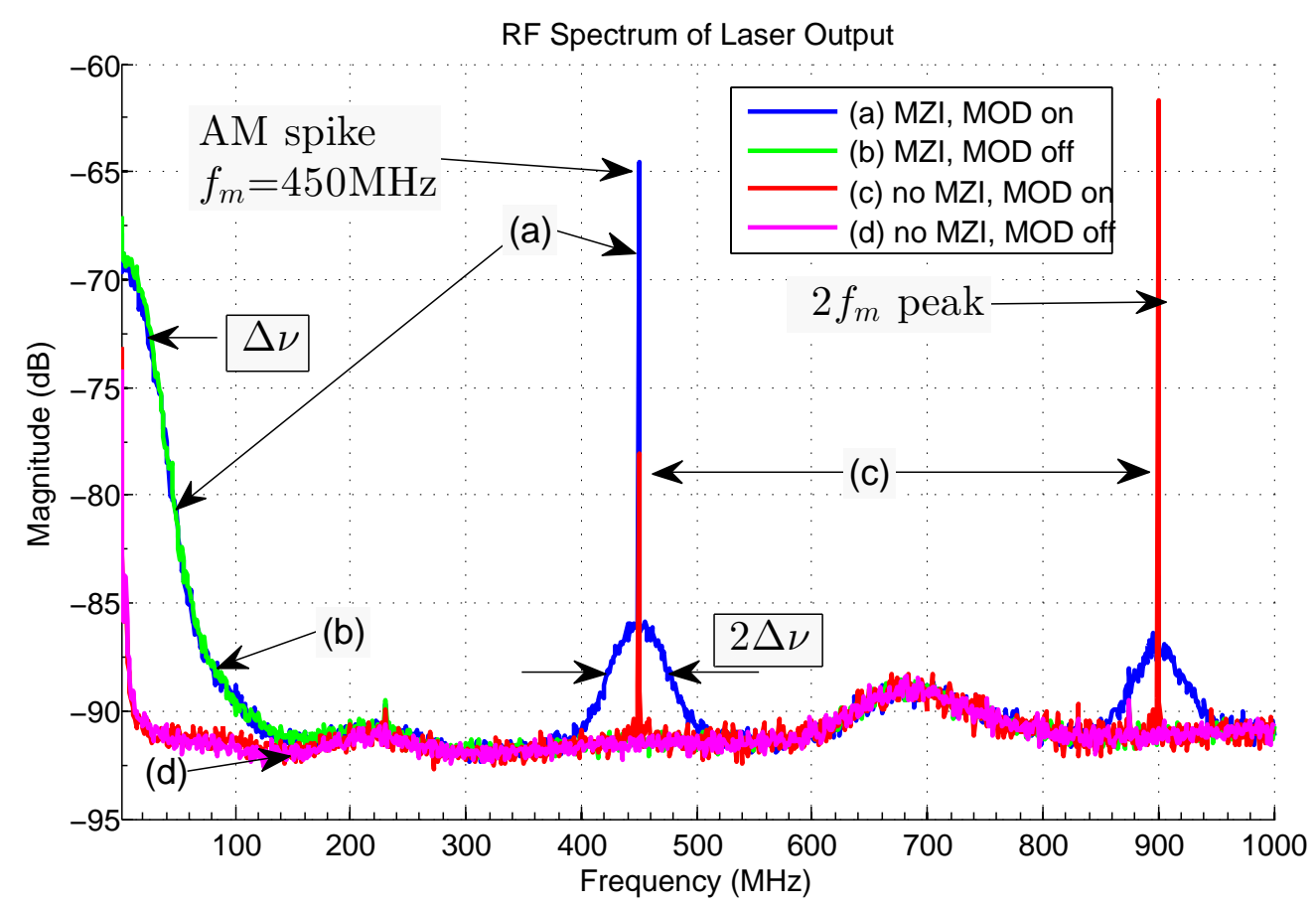

Figure 8.6: Two Sided Linewidth Measurement. (a) MZI coupled with modulation; (b) MZI coupled with modulation off; (c) MZI decoupled with modulation on; (d) MZI decoupled with no modulation; Modulation frequency $f_{m}=450 \mathrm{MHz}$ at $-4 \mathrm{dBm}$ into Gain section. $\mathrm{VBW}=$ $300 \mathrm{~Hz} ; \mathrm{RBW}=100 \mathrm{KHz}$; Span $[10 \mathrm{~K}-1 \mathrm{GHz}]$. Bias conditions of $I_{F M}$ $=0 \mathrm{~mA}, I_{B M}=0 \mathrm{~mA}, I_{P M}=0 \mathrm{~mA}, I_{S O A}=98 \mathrm{~mA}$, and $I_{G A I N}=100$ $\mathrm{mA}$. Wavelength under test $\lambda=1318.617 \mathrm{~nm}$. Single-sided linewidth is $23.0 \mathrm{MHz}$, where the dual-sided measurement is $24.2 \mathrm{MHz}$.

Linewidths are highly dependent upon biasing conditions. Through the use of the front and back mirror biasing matrix presented in figure 8.3, results indicate linewidth performance of generally less than $200 \mathrm{MHz}$ in the worst case scenarios. Individual tests isolated on adjusting the front mirror bias only indicate approximately $175 \mathrm{MHz}$ or less. Similar tests on the back mirror reveal generally less than $100 \mathrm{MHz}$ linewidth for most lower bias currents, with the linewidth increasing up to approximately $300 \mathrm{MHz}$ for higher currents between 16-20 mA. 


\section{Chapter 9}

\section{Conclusion and Future Work}

This thesis demonstrates several critical performance metrics of the Insight Photonics $1310 \mathrm{~nm}$ SG-DBR laser for use in optical coherence tomography applications. A testing fixture acting as a general purpose breakout for the packaged laser was successfully created to aid in rapidly testing additional lasers by implementing $50 \Omega$ microstrip breakouts. A fully integrated thermal electric cooler control circuit with an analog PID loop on the testing fixture properly regulates the on board laser die temperature.

Equivalent circuit models in chapter 5 for all five ports of the laser were characterized via time domain reflectometry. Simple small signal models of the diode structure allow for an estimation RC parasitic limited bandwidth. Table 9.1 summarizes the equivalent circuit models for each section.

From these equivalent circuit models, the RC limited bandwidth is calculated as shown in table 9.2. Several different key biasing currents are presented for each section with their corresponding $-3 \mathrm{~dB}$ bandwidth, as derived from the models. Intuitively as the bias current is increased, the dynamic resistance of the model 
decreases. Thus with an assumed constant capacitance the RC limited bandwidth much increase as bias current increases. Knowledge of the $\mathrm{RC}$ equivalent circuit models are useful when designing interfaces to the laser packaging, and optimizing the driving circuitry for each port.

Table 9.1: Equivalent Circuit Model Summary. Simple RC model is derived from TDR tests at specific bias currents.

\begin{tabular}{|c|c|c|c|c|c|c|c|c|c|}
\hline Parameter & \multicolumn{3}{|c|}{ Back } & \multicolumn{3}{|c|}{ Front } & \multicolumn{3}{|c|}{ Phase } \\
\hline \multirow{15}{*}{ Circuit Model } & $\begin{array}{c}I_{B M} \\
(\mathrm{~mA})\end{array}$ & $\begin{array}{c}\mathrm{C} \\
(\mathrm{pF})\end{array}$ & $\begin{array}{c}\mathrm{R} \\
(\Omega)\end{array}$ & $\begin{array}{c}I_{F M} \\
(\mathrm{~mA})\end{array}$ & $\begin{array}{c}\mathrm{C} \\
(\mathrm{pF})\end{array}$ & $\begin{array}{c}\mathrm{R} \\
(\Omega)\end{array}$ & $\begin{array}{c}I_{P M} \\
(\mathrm{~mA})\end{array}$ & $\begin{array}{c}\mathrm{C} \\
(\mathrm{pF})\end{array}$ & $\begin{array}{c}\mathrm{R} \\
(\Omega)\end{array}$ \\
\hline & 0 & 10.7 & $4.1 \mathrm{~K}$ & 0 & 5 & $4.9 \mathrm{~K}$ & 0 & 4 & $16 \mathrm{~K}$ \\
\hline & 5 & 10.7 & 25.4 & 5 & 5 & 32.3 & 5 & 4 & 46.9 \\
\hline & 10 & 10.7 & 9.5 & 10 & 5 & 19 & 7.5 & 4 & 37.1 \\
\hline & 20 & 10.7 & 7.3 & 20 & 5 & 14.5 & 10 & 4 & 32.8 \\
\hline & 40 & 10.7 & 6.4 & 30 & 5 & 11.8 & 15 & 4 & 25.9 \\
\hline & 60 & 10.7 & 5.9 & 35 & 5 & 10.9 & 20 & 4 & 21.9 \\
\hline & \multicolumn{3}{|c|}{ Gain } & \multicolumn{3}{|c|}{$\mathrm{SOA}$} & \multicolumn{3}{|c|}{ RC Model } \\
\hline & $\begin{array}{c}I_{G A I N} \\
(\mathrm{~mA})\end{array}$ & $\begin{array}{c}\mathrm{C} \\
(\mathrm{pF})\end{array}$ & $\begin{array}{c}\mathrm{R} \\
(\Omega)\end{array}$ & $\begin{array}{l}I_{S O A} \\
(\mathrm{~mA})\end{array}$ & $\begin{array}{c}\mathrm{C} \\
(\mathrm{pF})\end{array}$ & $\begin{array}{c}\mathrm{R} \\
(\Omega)\end{array}$ & \multicolumn{3}{|c|}{ ㅇ } \\
\hline & 0 & 9 & $6.2 \mathrm{~K}$ & 0 & 7 & $16 \mathrm{~K}$ & & & \\
\hline & 10 & 9 & 15.2 & 10 & 7 & 17.1 & & & $=C$ \\
\hline & 40 & 9 & 8.8 & 50 & 7 & 8.8 & & & \\
\hline & 100 & 9 & 5.9 & 100 & 7 & 6.5 & & & \\
\hline & 125 & 9 & 5.5 & 125 & 7 & 5.8 & & 0 & \\
\hline & 140 & 9 & 5.3 & 147 & 7 & 5.3 & & & \\
\hline
\end{tabular}

Amplitude modulation tests of the gain and semiconductor optical amplifier (SOA) sections of the laser determine the maximum effective intensity modulation rate. This measurement simultaneously investigates the $\mathrm{RC}$ limited bandwidth, primarily present at lower bias currents. In table 9.3 the amplitude modulation response tests are summarized. Several bias conditions on the Gain and SOA sections are measured and the $-3 \mathrm{~dB}$ point obtained.

Recall from the previous table 9.2 , which presents the RC limited bandwidth estimations. Comparing to the amplitude modulation response table for the Gain 
Table 9.2: RC Limited Bandwidth Summary. Bias current for each section and the corresponding bandwidth (BW) is compared.

\begin{tabular}{|c||cc|cc|cc|cc|cc|}
\hline \multicolumn{1}{|c||}{ Parameter } & \multicolumn{2}{c|}{ Back } & \multicolumn{2}{c|}{ Front } & \multicolumn{2}{c|}{ Phase } & \multicolumn{2}{c|}{ Gain } & \multicolumn{2}{c|}{ SOA } \\
\hline \hline \multirow{5}{*}{ RC Limited } & $I_{B M}$ & $\mathrm{BW}$ & $I_{F M}$ & $\mathrm{BW}$ & $I_{P M}$ & $\mathrm{BW}$ & $I_{G A I N}$ & $\mathrm{BW}$ & $I_{S O A}$ & $\mathrm{BW}$ \\
& $\mathrm{mA}$ & $\mathrm{MHz}$ & $\mathrm{mA}$ & $\mathrm{MHz}$ & $\mathrm{mA}$ & $\mathrm{MHz}$ & $\mathrm{mA}$ & $\mathrm{MHz}$ & $\mathrm{mA}$ & $\mathrm{MHz}$ \\
\cline { 2 - 12 } & 5 & 585 & 5 & 985 & 5 & 848 & 10 & 1163 & 10 & 1329 \\
& 10 & 1565 & 10 & 1675 & 7.5 & 1072 & 40 & 2009 & 50 & 2583 \\
& 20 & 2037 & 20 & 2195 & 10 & 1213 & 100 & 2997 & 100 & 3497 \\
& 40 & 2324 & 30 & 2697 & 15 & 1536 & 125 & 3215 & 125 & 3920 \\
& 60 & 2521 & 35 & 2920 & 20 & 1816 & 140 & 3336 & 147 & 4289 \\
\hline
\end{tabular}

section, it is clear that the relaxation oscillations limit the effective modulation bandwidth at moderate to high bias currents, well before the RC parasitic limitations take into effect. In general for small bias currents, especially when the laser is operating below and near threshold, the RC parasitics limit the bandwidth. For all practical operating purposes, the laser would be operated well above threshold, therefore $\mathrm{RC}$ parasitic induced limitations are not of a concern in the overall bandwidth limitation. The optimal bias currents for the Gain section are approximately $80-100 \mathrm{~mA}$, to achieve $>1.5 \mathrm{GHz}$ modulation bandwidth.

Since the SOA is not inside the cavity of the laser, it does not experience the relaxation oscillation limitations. The response of this section is much more stable over bias currents compared to the Gain section. In order to achieve $>500 \mathrm{MHz}$ modulation bandwidth, bias currents of $60-100 \mathrm{~mA}$ are required.

Frequency modulation of the three frequency tuning elements of the Front, Back, and Phase sections sets the maximum sweep rate for wavelength tuning, an essential property for source swept optical coherence tomography. Table 9.4 provides a summary of the approximate frequency modulation bandwidths for the Front, Back, and Phase sections.

For the Back mirror section, the bias was set to $27.3 \mathrm{~mA}$ and fixed, while the 
Table 9.3: Amplitude Modulation Response Summary. Gain and SOA sections and the respective bias conditions are presented. Optimal Gain section bias of $80-100 \mathrm{~mA}$ for a bandwidth $>1.5 \mathrm{GHz}$. For the SOA section, the optimal bias is $60-100 \mathrm{~mA}$ for $>500 \mathrm{MHz}$ bandwidth.

\begin{tabular}{|c||cc|cc|}
\hline Parameter & \multicolumn{2}{c|}{ Gain } & \multicolumn{2}{c|}{ SOA } \\
\hline \hline \multirow{5}{*}{} & $I_{\text {GAIN }}$ & BW & $I_{\text {SOA }}$ & BW \\
& $(\mathrm{mA})$ & $(\mathrm{MHz})$ & $(\mathrm{mA})$ & $(\mathrm{MHz})$ \\
\cline { 2 - 5 } & 50 & 120 & 5 & 110 \\
& 55 & 210 & 7.3 & 200 \\
& 57 & 340 & 10 & 280 \\
& 58 & 930 & 21.2 & 290 \\
& 60 & 1370 & 31 & 370 \\
& 61.9 & 1580 & 39.9 & 390 \\
& 64.3 & 1880 & 50.5 & 450 \\
& 66.5 & 610 & 60.5 & 520 \\
& 68.7 & 340 & 70.8 & 520 \\
& 70.6 & 220 & 80.7 & 520 \\
& 73 & 440 & 100 & 510 \\
& 75 & 910 & 110 & 450 \\
& 80.6 & 1900 & 125 & 460 \\
& 85.2 & 1800 & 150 & 450 \\
& 90.2 & 1850 & 168 & 450 \\
& 95 & 2690 & & \\
& 100 & 150 & & \\
& 110 & 230 & & \\
& 120 & 180 & & \\
\hline
\end{tabular}


phase was used to fully tune within the FSR of the interferometer. A modulation bandwidth of $110 \mathrm{MHz}$ was determined from the average of several quadrature points on the Back mirror.

Similarly to the Back mirror, the Front mirror was biased at a fixed $12.3 \mathrm{~mA}$, while the phase current was used to tune the frequency. The Front mirror section had a bandwidth of approximately $180 \mathrm{MHz}$, as averaged from several quadrature lock points.

The phase section had both mirrors biased at a fixed $0 \mathrm{~mA}$, while the phase injection current was varied. Between several trial runs of frequency modulation on the phase section, ranging from $5.3-8.2 \mathrm{~mA}$, the average effective bandwidth was $250 \mathrm{MHz}$.

Table 9.4: Frequency Modulation Response Summary. The Front, Back, and Phase sections maximum modulation bandwidth and the respective bias conditions are presented. ${ }^{2}$

\begin{tabular}{|c||cc|cc|cc|}
\hline \multicolumn{1}{|c||}{ Parameter } & \multicolumn{2}{c}{ Back } & \multicolumn{2}{c|}{ Front } & \multicolumn{2}{c|}{ Phase } \\
\hline \hline \multirow{5}{*}{ FM Response } & $I_{B M}$ & $\begin{array}{c}\mathrm{BW} \\
(\mathrm{mA})\end{array}$ & $I_{F M}$ & $\mathrm{BW}$ & $I_{P M}$ & $\mathrm{BW}$ \\
& 27.3 & 110 & 12.3 & 180 & 5.3 & 214 \\
& & & & & 5.8 & 223 \\
& & & & & 7.6 & 278 \\
& & & & & 8.2 & 283 \\
\hline
\end{tabular}

Finally the linewidth mapping determines the spectral purity of the laser with respect to bias current. For linewidth measurements the bias current of the mirrors greatly influences the performance. Observations indicate solely varying back mirror currents yield linewidth less than $100 \mathrm{MHz}$ for most lower bias cur-

\footnotetext{
${ }^{2}$ Frequency Modulation testing procedure: The Back and Front mirror tests utilize a fixed bias, while tuning within the FSR is achieved via varying Phase injection current. Therefore the presented value for the Back and Front is an average of the quadrature points for the specific bias. The Phase section is independently tuned with all other sections fixed at $0 \mathrm{~mA}$. The Phase section bias points are recorded for each quadrature polarity, for several runs. In each test $I_{S O A}=98 \mathrm{~mA}$, and $I_{G A I N}=100 \mathrm{~mA}$
} 
rents. However when biased higher from 16-20 mA, the back mirror linewidths increased to approximately $300 \mathrm{MHz}$. Only adjusting the front mirror bias provides linewidths of $175 \mathrm{MHz}$ or less. A more detailed 2D investigation into simultaneously varying the front and back mirror currents reveals the best performance of $26 \mathrm{MHz}$ at the origin. Sweeping the front mirror currents up to $5 \mathrm{~mA}$, and the back mirror currents up to $7 \mathrm{~mA}$, further worsens the linewidth performance, maximizing at approximately $200 \mathrm{MHz}$.

Additional research includes a full mode mapping of the $1310 \mathrm{~nm}$ laser, to investigate the mode pattern and develop an automated tuning profile. Testing of more mature lasers besides these early prototypes would be beneficial for greater understanding and comparison of the performance. Investigation into the FM induced laser chirp and additional measurements of the laser's power spectrum would be valuable in conjunction with AM and FM bandwidths. Integration of automated data collection is essential for characterizing additional lasers, as the current manual tuning and acquisition is very time consuming. A finer resolution linewidth mapping profile would also be beneficial once the lasers are more mature. A more in depth testing of the thermal electric cooler for any possible noise sources on the laser would be useful for eventual product integration.

In conclusion, this thesis investigated the modeling and characterization of an early generation $1310 \mathrm{~nm}$ O-Band SG-DBR laser from Insight Photonics. The equivalent circuit models, amplitude modulation, frequency modulation, and linewidth performance was quantified for ultimately determining the limits of operation in optical coherence tomography applications. 


\section{Bibliography}

[1] D. Huang, E. A. Swanson, C. P. Lin, J. S. Schuman, W. G. Stinson, W. Chang, M. R. Hee, T. Flotte, K. Gregory, C. A. Puliafito, and J. G. Fujimoto, "Optical coherence tomography," Science, vol. 254, no. 5035, pp. pp. 1178-1181, 1991.

[2] Z. Yaqoob, J. Wu, and C. Yang, "Spectral domain optical coherence tomography: a better oct imaging strategy," BioTechniques, 2005.

[3] University of Western Australia, "Introduction to Optical Coherence Tomography (OCT)," Optical+Biomedical Engineering Laboratory, 2013.

[4] Santec Corporation, "OCT Solutions," vol. 1.1, 2010.

[5] B. Zabihian, M. Minneman, M. Bonesi, J. Ensher, H. Sattmann, S. Gray, R. Leitgeb, M. Crawford, and W. Drexler, "Akinetic swept-source technology for high speed oct with unprecedented coherence length," in Bio-Optics: Design and Application, Optical Society of America, 2013.

[6] G. P. Agrawal and N. K. Dutta, "Long wavelength semiconductor lasers," 1986.

[7] W. T. Silfvast, Laser fundamentals. Cambridge University Press, 2004.

[8] O. Svelto, Principles of lasers. Springer, 2010. 
[9] B. E. Saleh, M. C. Teich, and B. E. Saleh, Fundamentals of photonics, vol. 22. Wiley New York, 1991.

[10] J. C. Palais, Fiber optic communications. Prentice Hall, 2004.

[11] M. C. Larson, Y. A. Akulova, C. W. Coldren, T. Liljeberg, G. A. Fish, S. Nakagawa, A. Dahl, P. Kozodoy, D. Bingo, M. Bai, et al., "High performance widely-tunable sg-dbr lasers," in Integrated Optoelectronics Devices, pp. 66-80, International Society for Optics and Photonics, 2003.

[12] B. J. George, "Swept-frequency sampled grating distributed bragg reflector lasers optimized for optical coherence tomography applications," 2009.

[13] C. L. Chiccone, "Automated SG-DBR tunable laser calibration optimized for optical coherence tomography applications," 2011.

[14] S. O'Connor, High Speed Wavelength Tuning of SG-DBR Lasers for Light Detection and Ranging and Optical Coherence Tomography: A Thesis. PhD thesis, California Polytechnic State University, 2008.

[15] "Avanced Circuits." http : //www.4pcb.com/33-each - pcbs/.

[16] "Isola Group FR402." http : //www.isola - group.com/products/FR402/.

[17] "SMA 50 Ohm Receptacle Datasheet." https : //emersonconnectivitysolutions.com/OA $A_{M}$ EIA/specs/pi - 142 $0701-851 . p d f$.

[18] "LASORB ESD absorber Background Information." https : //emersonconnectivitysolutions.com/OA $A_{M}$ DIA/specs/pi - 142 0701 - 851.pdf. 
[19] "TEC definition." http ://searchnetworking.techtarget.com/definition/thermoelectric - cooling.

[20] "Maxim MAX1968 Datasheet." http : //www.maximintegrated.com/ datasheet/index.mvp/id/3377.

[21] M. F. Iskander, Electromagnetic fields and waves. Prentice Hall Englewood Cliffs, 1992.

[22] M.-B. Su and Y.-J. Chen, "Multiple reflection of metallic time domain reflectometry," Experimental Techniques, vol. 22, no. 1, pp. 26-29, 1998.

[23] S. Charles and K. Jing, "Mit open courseware: Microelectronic devices and circuits (pdf)," 2013.

[24] "Wirebond impedance and attenuation." http : //www.microwaves101.com/encyclopedia/Inductance ${ }_{w}$ irebond.cfm.

[25] D. Derickson and C. Hentschel, Fiber optic test and measurement. Prentice Hall PTR New Jersey, 1998.

[26] J. Bowers, "High speed semiconductor laser design and performance," Solidstate electronics, vol. 30, no. 1, pp. 1-11, 1987.

[27] "Rp photonics - semiconductor optical amplifiers." http : //www.rp photonics.com/semiconductor ${ }_{\text {optical }}$ mplifiers.html.

[28] D. Baney, P. Gallion, and C. Chabran, "Measurement of the swept-frequency carrier-induced fm response of a semiconductor laser using an incoherent interferometric technique," Photonics Technology Letters, IEEE, vol. 2, no. 5, pp. 325-327, 1990. 
[29] W. V. Sorin, K. W. Chang, G. A. Conrad, and P. R. Hernday, "Frequency domain analysis of an optical fm discriminator," Lightwave Technology, Journal of, vol. 10, no. 6, pp. 787-793, 1992.

[30] J. Wilson and J. F. Hawkes, "Optoelectronics-an introduction," 1989.

[31] "Rp photonics - linewidth." http : / /www.rp photonics.com/linewidth.html.

[32] D. Baney and W. Sorin, "Linewidth and power spectral measurements of single-frequency lasers," Hewlett-Packard Journal, vol. 41, pp. 92-6, 1990.

[33] R. Esman and L. Goldberg, "Simple measurement of laser diode spectral linewidth using modulation sidebands," Electronics Letters, vol. 24, no. 22, pp. 1393-1395, 1988. 


\section{Appendices}

\section{A Laser Pinout}

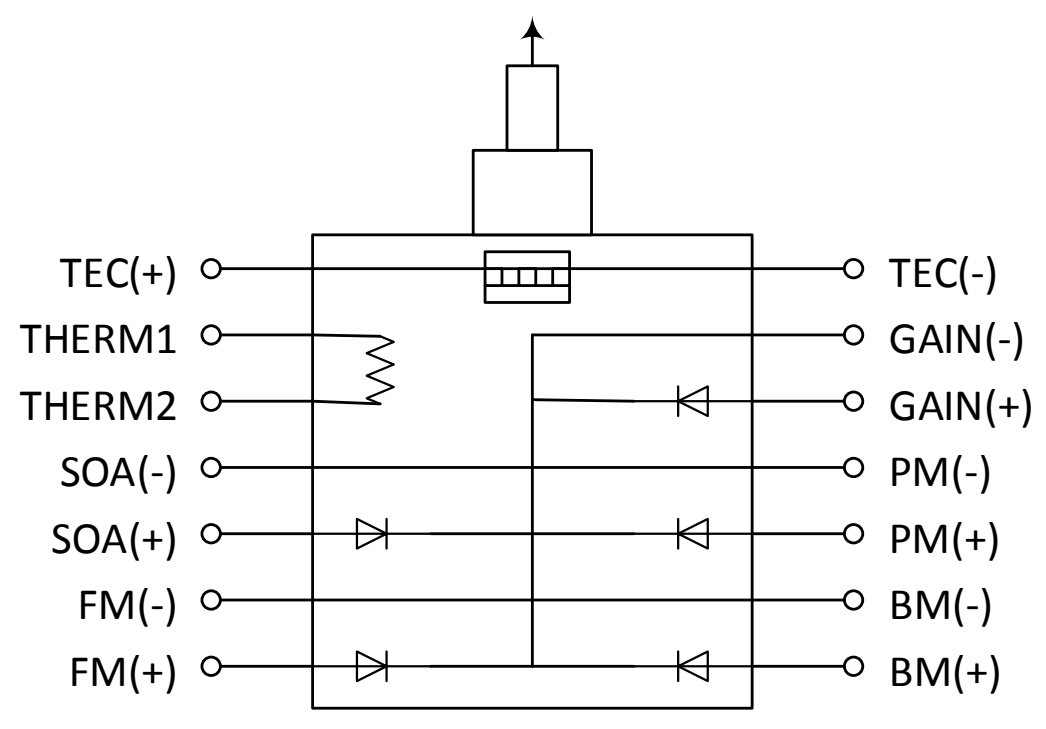

Figure A.1: Laser Pinout Diagram. Common cathode diode segments. Thermistor 10K @ $25^{\circ} \mathrm{C}$. TEC 1.5 A max current. 


\section{B TEC Controller Schematic}

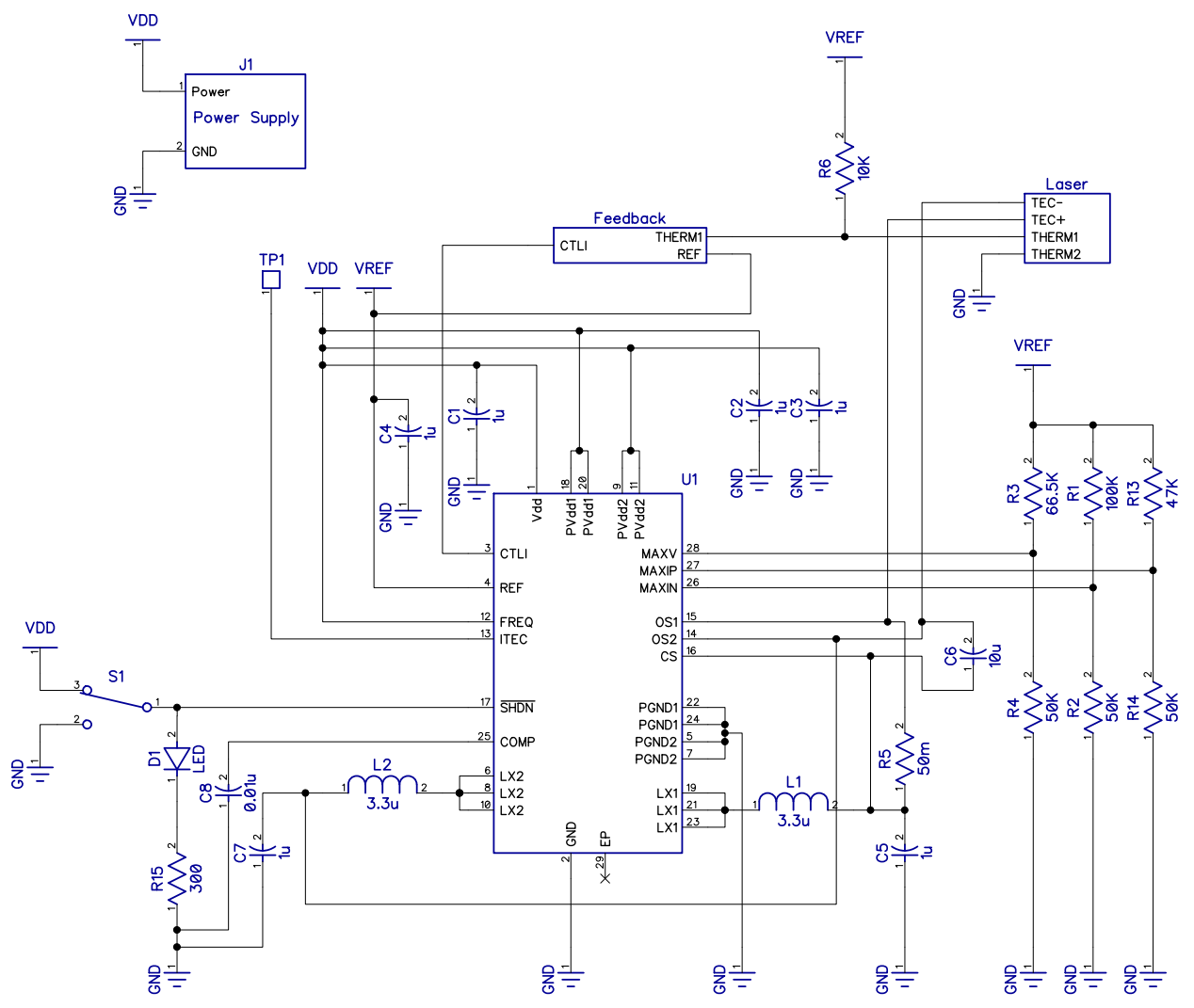

Figure B.1: Schematic Main Controller, Maxim1968 TEC Controller 




Figure B.2: Schematic PID Loop

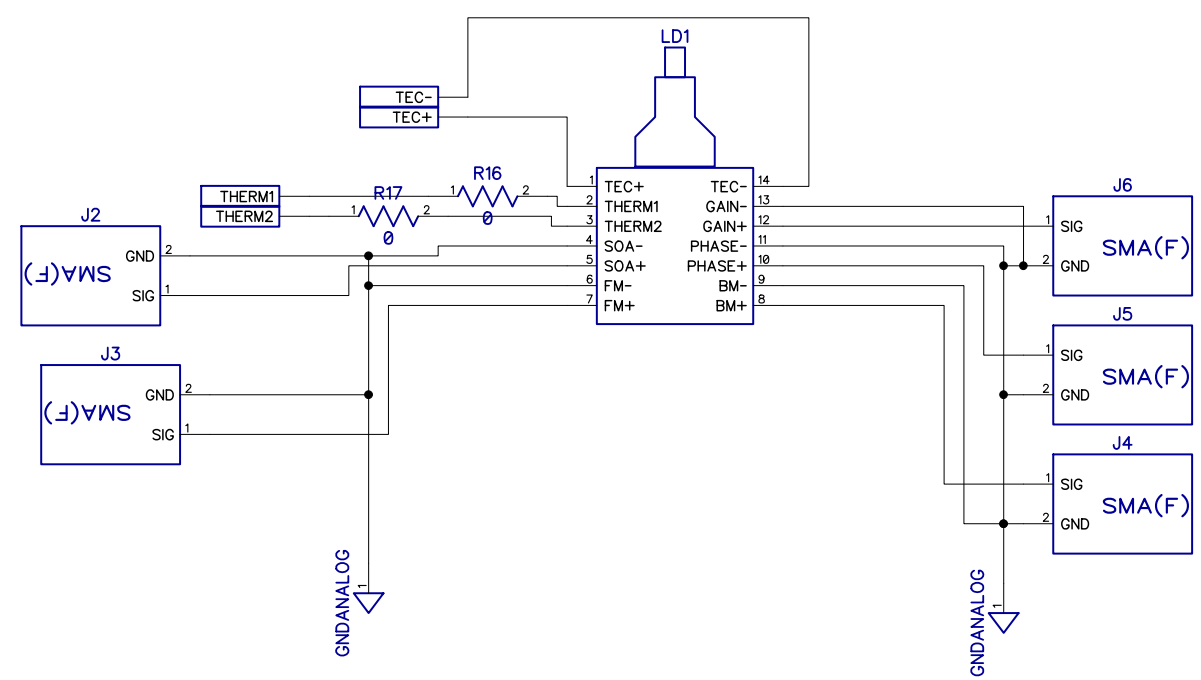

Figure B.3: Schematic Laser Connection 


\section{PCB Gerber Files}

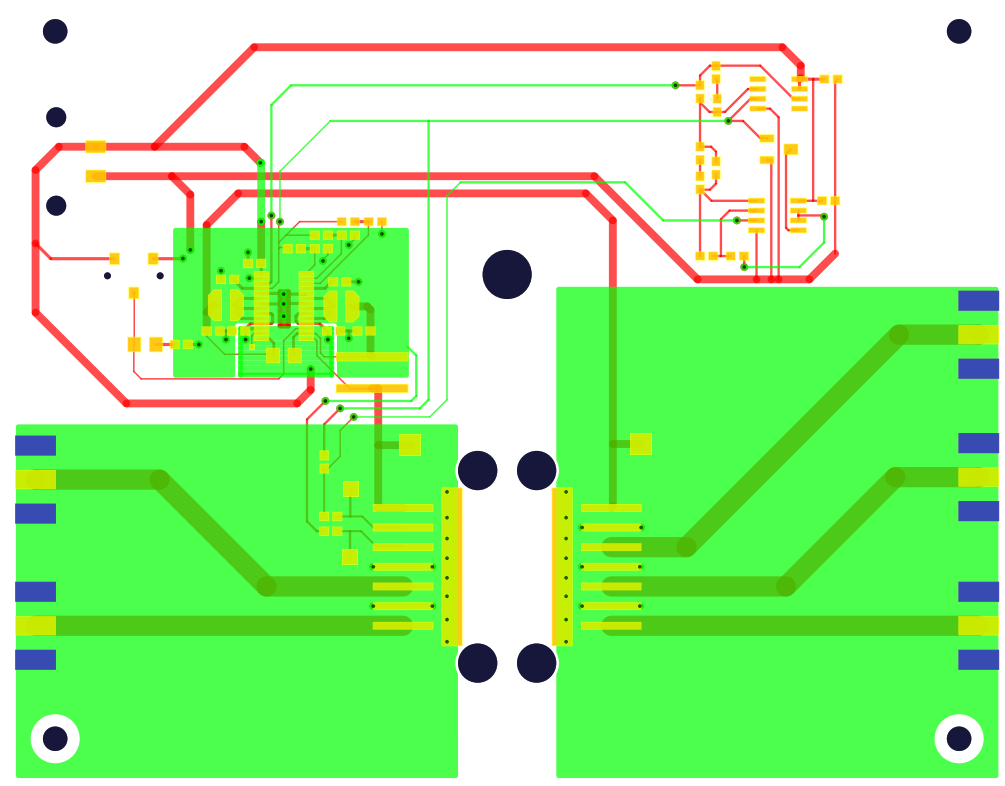

Figure C.1: PCB All Layer Composite

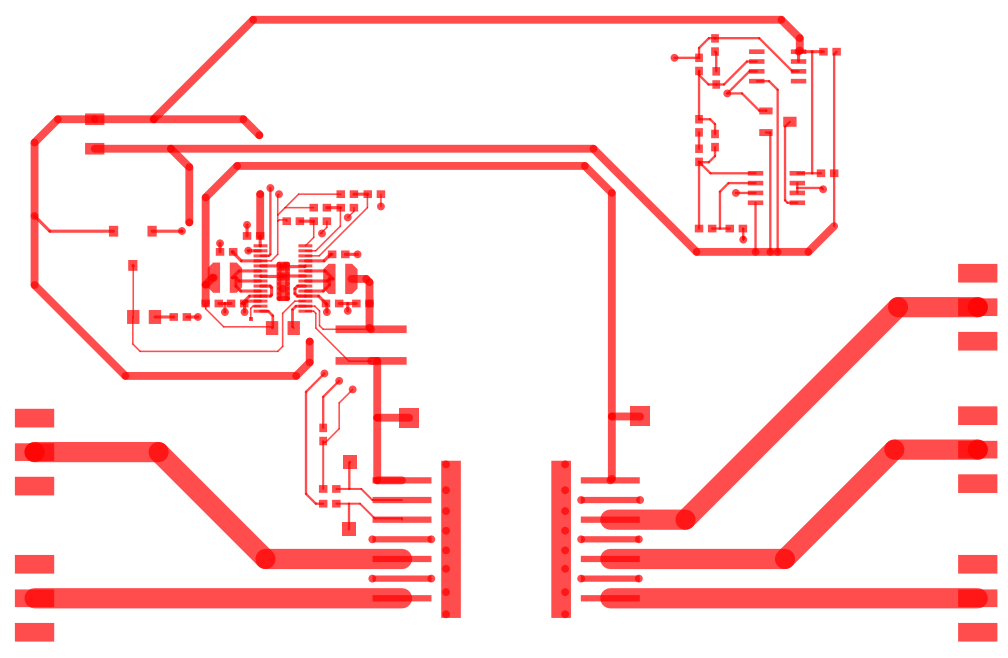

Figure C.2: PCB Top Copper Layer 


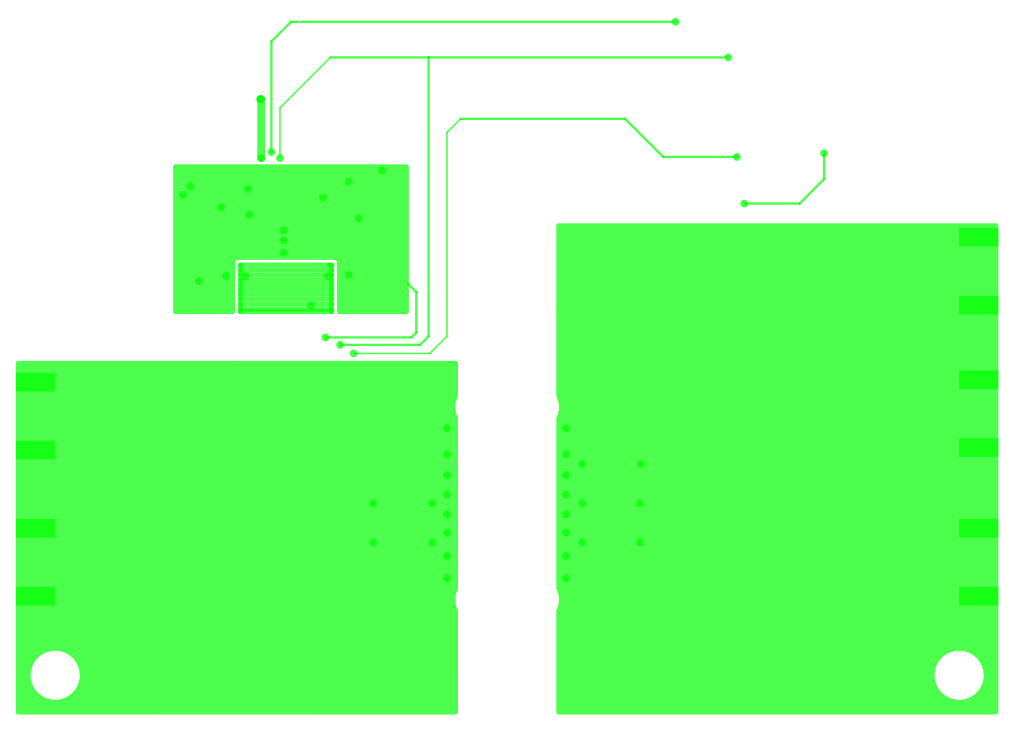

Figure C.3: PCB Bottom Copper Layer

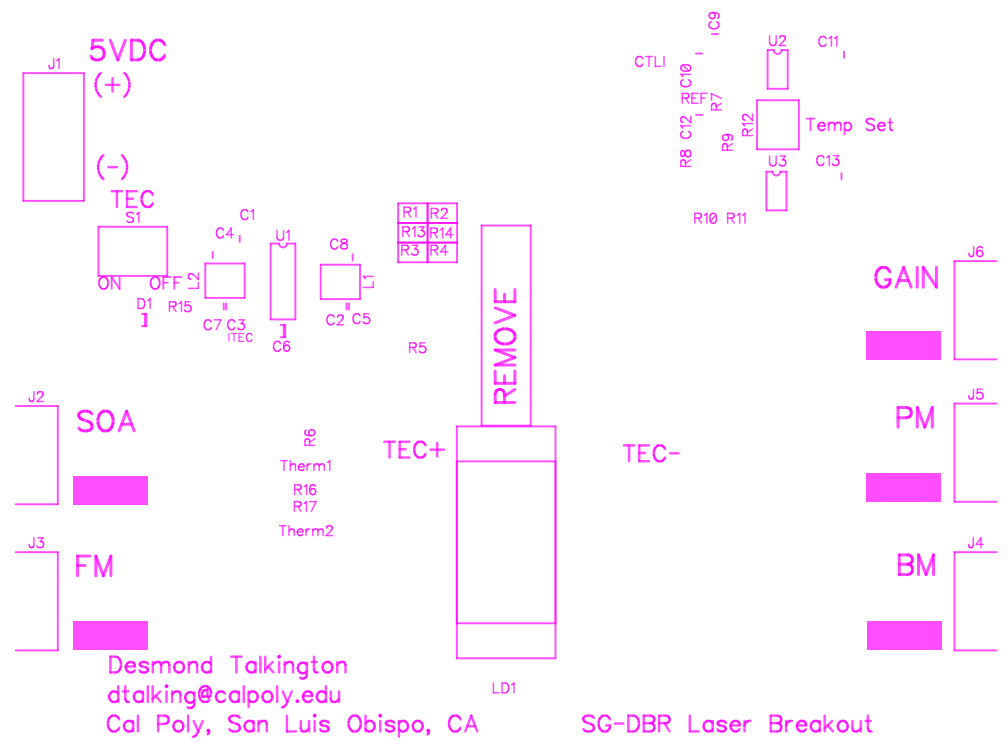

Figure C.4: PCB Top Assembly Layer 


\section{Bill of Materials}

The complete electrical bill of materials (BOM) is presented below for the testing fixture board and support circuitry. The majority of the parts are acquired from Digikey, with the exception for the SMA connectors obtained from Allied Electronics. Samples may be obtained for the Maxim 1968 TEC controller from their website. 


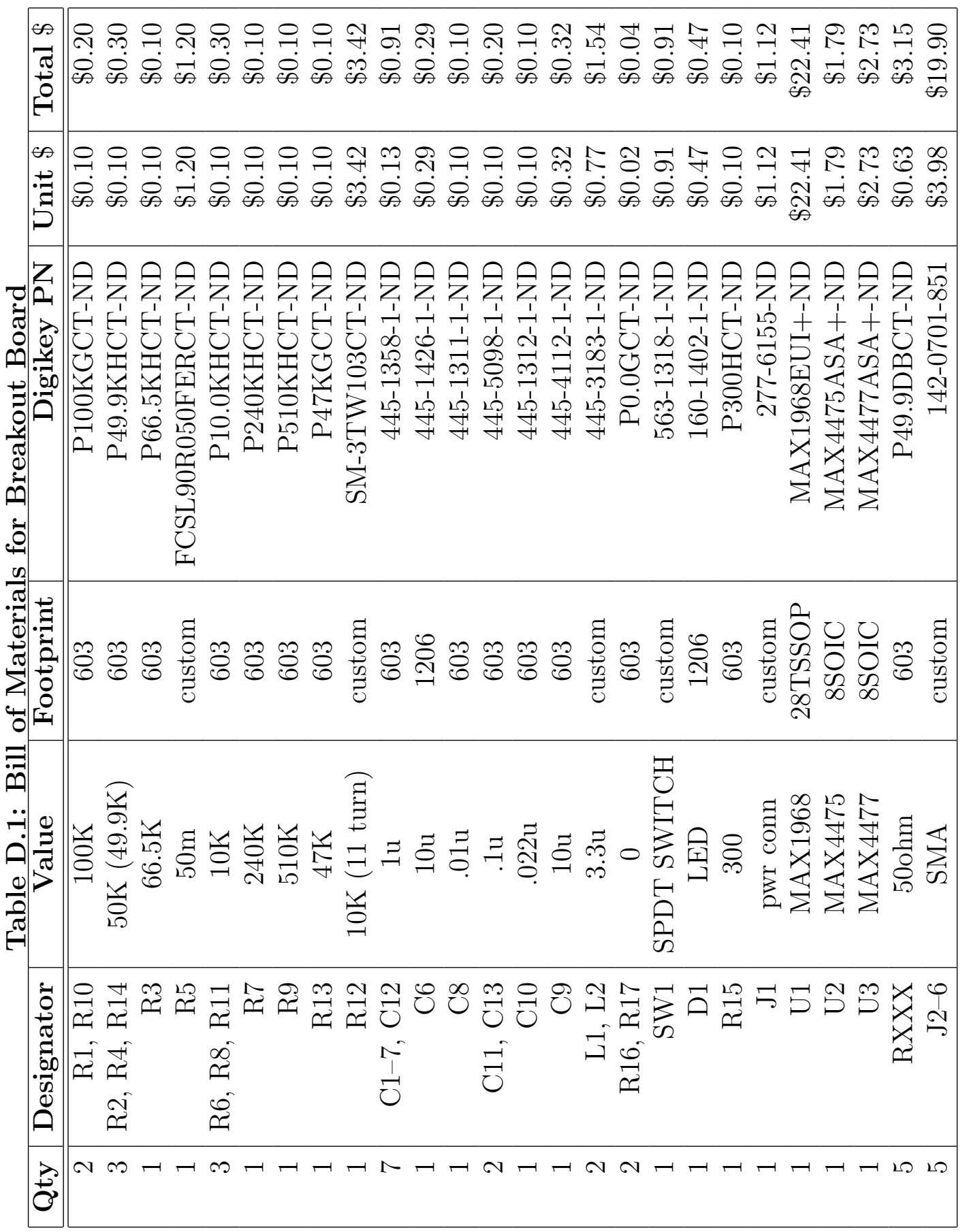




\section{E Amplitude Modulation Data}

The raw $\left(S_{21}\right)$ captures from the amplitude modulation of gain sections are presented below in figure E.1. Refer to section 6 in the results chapter for the complete analysis of the modulation with respect to biasing current. Figure E.2 is the complete trial run of SOA bias currents with the Gain $=100 \mathrm{~mA}$ fixed, all others held constant.

Data is extracted from tabular data export from the Anritsu VNA and plotted via MATLAB. The $-3 \mathrm{~dB}$ frequencies and global maximums are automatically located and printed in the information box of the plot. 


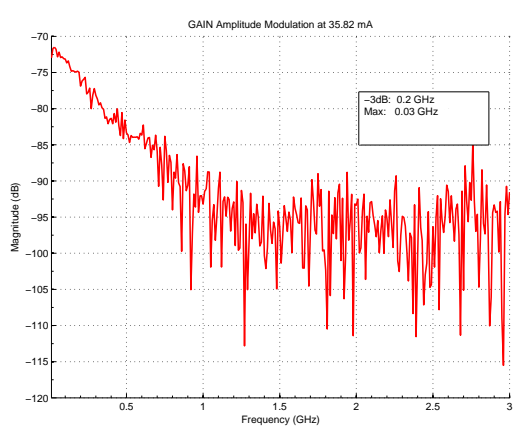

(a) $I_{\text {GAIN }}=35.82 \mathrm{~mA}$

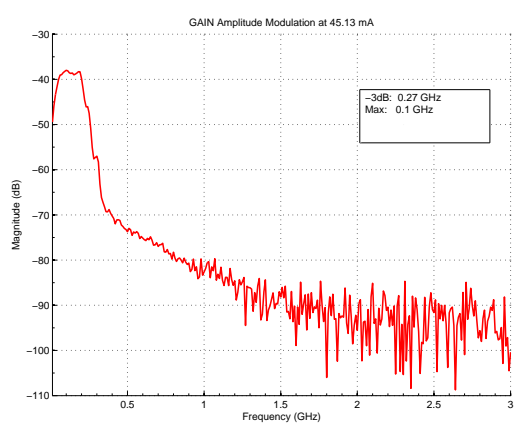

(c) $I_{\text {GAIN }}=45.13 \mathrm{~mA}$

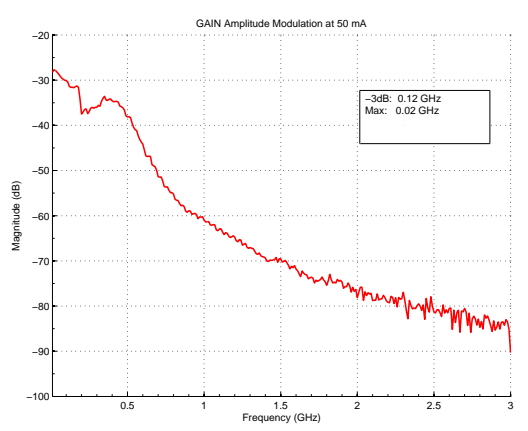

(e) $I_{\text {GAIN }}=50.0 \mathrm{~mA}$

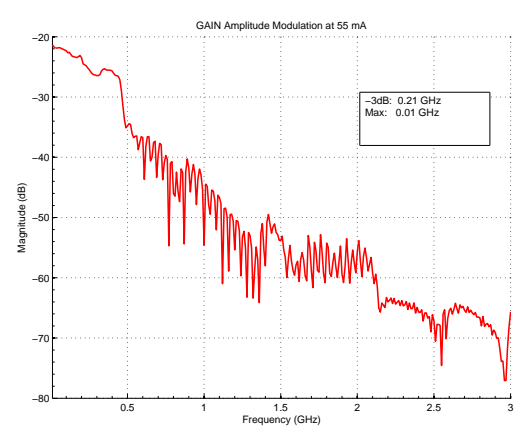

(g) $I_{G A I N}=55.0 \mathrm{~mA}$



(b) $I_{\text {GAIN }}=43.0 \mathrm{~mA}$

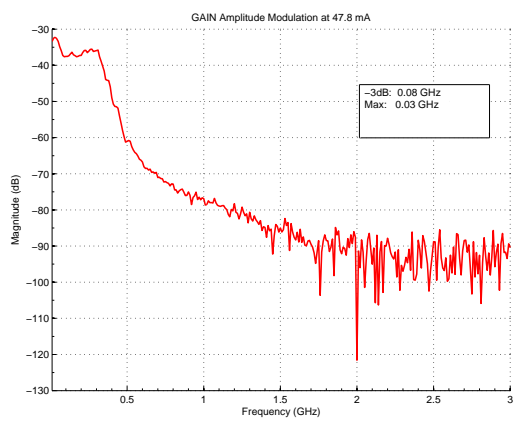

(d) $I_{\text {GAIN }}=47.8 \mathrm{~mA}$



(f) $I_{\text {GAIN }}=53.0 \mathrm{~mA}$

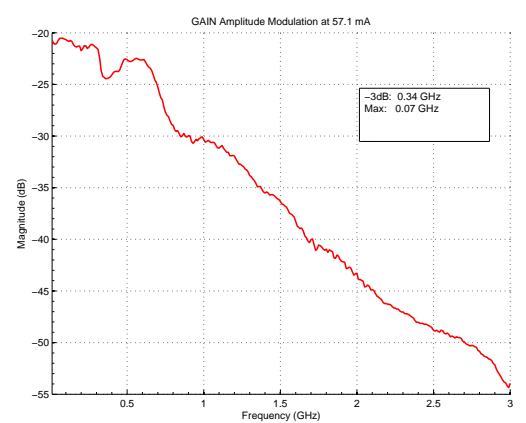

(h) $I_{\text {GAIN }}=57.1 \mathrm{~mA}$ 


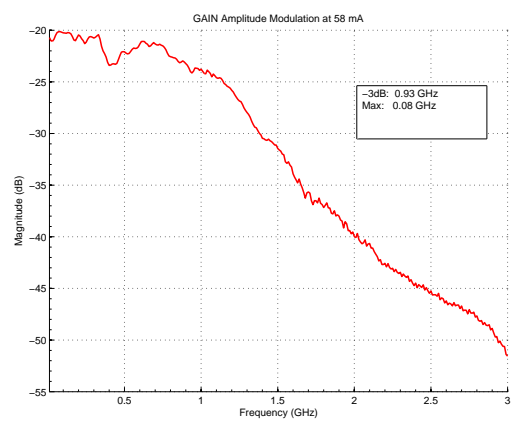

(i) $I_{G A I N}=58.0 \mathrm{~mA}$

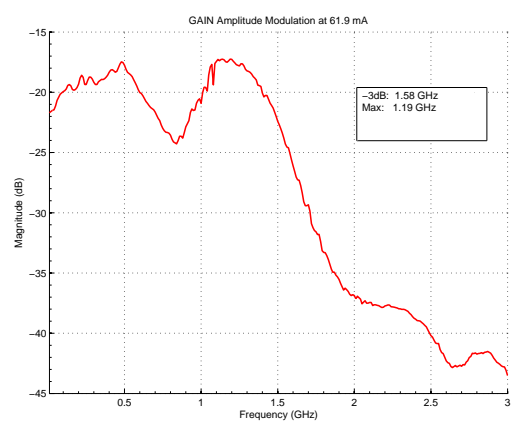

(k) $I_{G A I N}=61.9 \mathrm{~mA}$

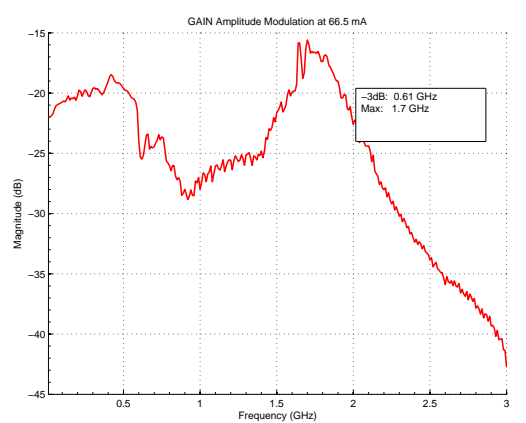

(m) $I_{\text {GAIN }}=66.5 \mathrm{~mA}$

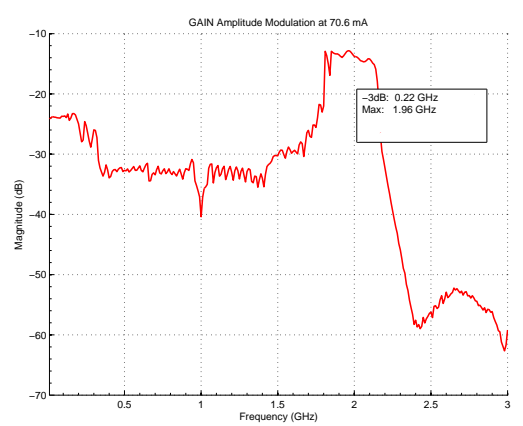

(o) $I_{\text {GAIN }}=70.6 \mathrm{~mA}$

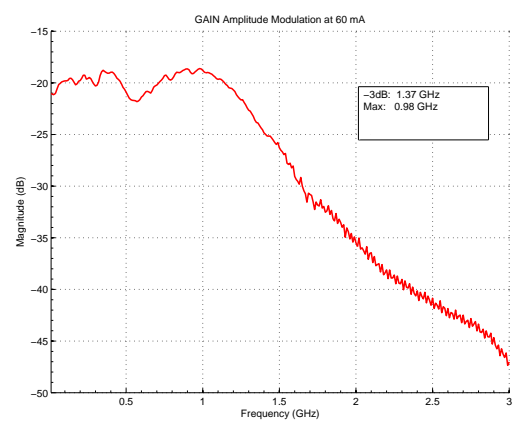

(j) $I_{\text {GAIN }}=60.0 \mathrm{~mA}$

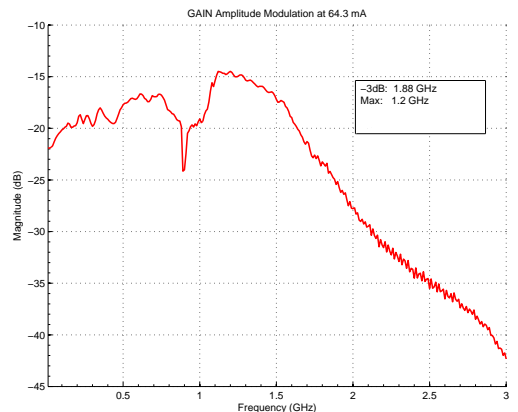

(l) $I_{\text {GAIN }}=64.3 \mathrm{~mA}$



(n) $I_{\text {GAIN }}=68.7 \mathrm{~mA}$

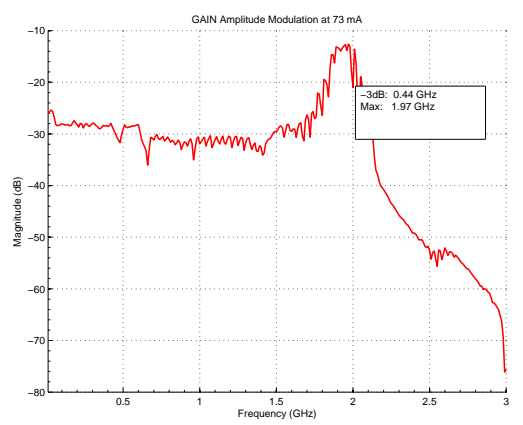

(p) $I_{\text {GAIN }}=73.0 \mathrm{~mA}$ 


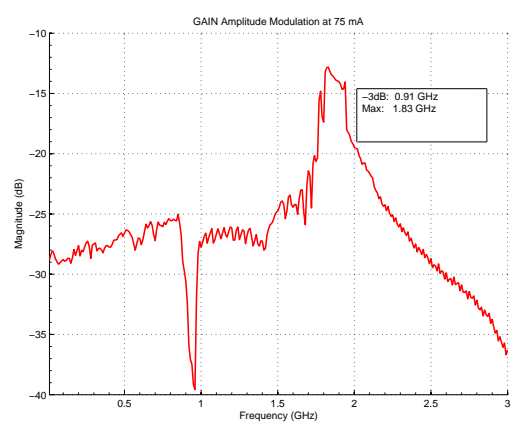

(q) $I_{G A I N}=75.0 \mathrm{~mA}$

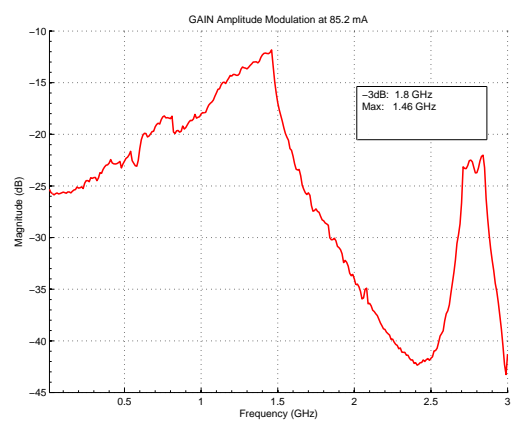

(s) $I_{\text {GAIN }}=85.2 \mathrm{~mA}$

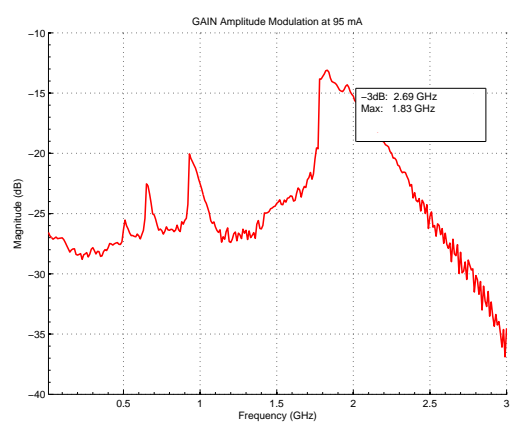

(u) $I_{\text {GAIN }}=95.0 \mathrm{~mA}$



(w) $I_{\text {GAIN }}=110.0 \mathrm{~mA}$



(r) $I_{G A I N}=80.6 \mathrm{~mA}$

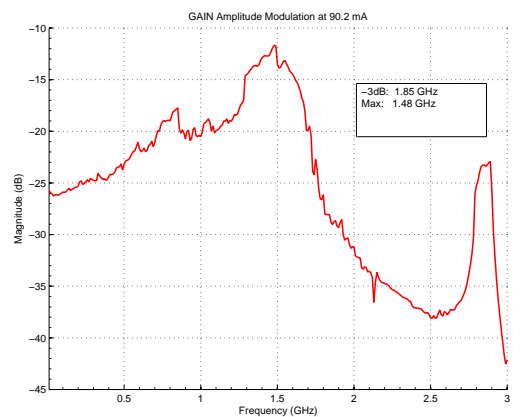

(t) $I_{\text {GAIN }}=90.2 \mathrm{~mA}$



(v) $I_{\text {GAIN }}=100.0 \mathrm{~mA}$

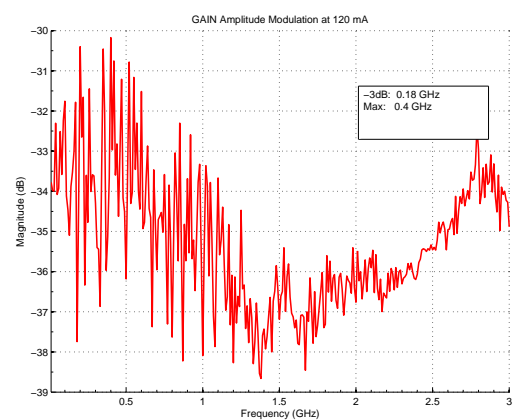

(x) $I_{\text {GAIN }}=120.0 \mathrm{~mA}$ 




(y) $I_{\text {GAIN }}=130.0 \mathrm{~mA}$

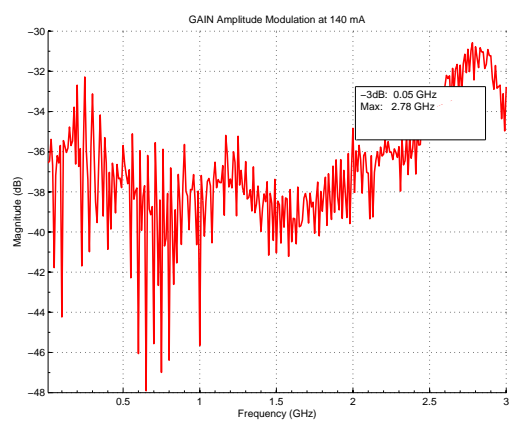

(z) $I_{\text {GAIN }}=140.0 \mathrm{~mA}$

Figure E.1: AM Response $\left(S_{21}\right)$ of Varying GAIN Bias. Current is varied from $I_{G A I N}=35.8-140 \mathrm{~mA}$ in $(\mathrm{a})-(\mathrm{z})$; Bias conditions are $I_{P M}$ $=I_{B M}=I_{F M}=0 \mathrm{~mA}$, and $I_{S O A}=98 \mathrm{~mA}$. The $-3 \mathrm{~dB}$ frequency as well as the location of the maximum amplitude peak are presented in the box of each subplot. The optimal bias is $80-100 \mathrm{~mA}$ for a bandwidth $>1.5 \mathrm{GHz}$. 


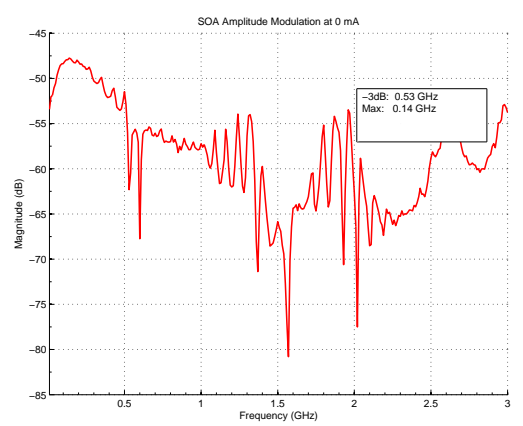

(a) $I_{S O A}=0.00 \mathrm{~mA}$

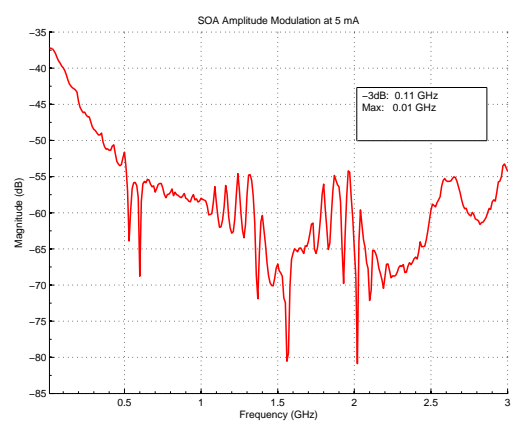

(c) $I_{S O A}=5.00 \mathrm{~mA}$

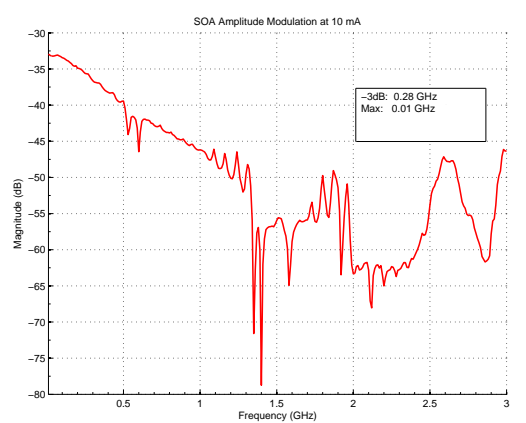

(e) $I_{S O A}=10.00 \mathrm{~mA}$

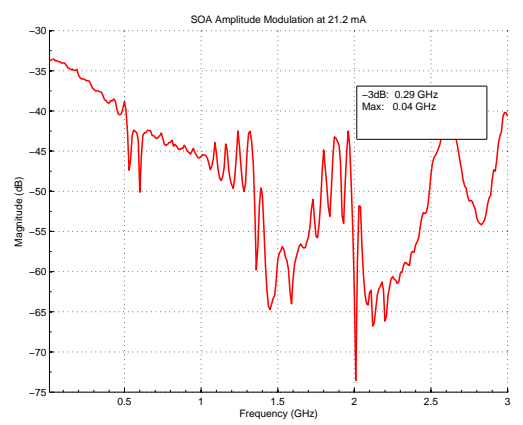

(g) $I_{S O A}=21.20 \mathrm{~mA}$

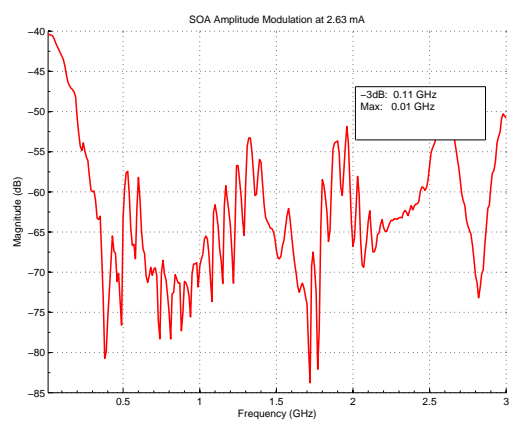

(b) $I_{S O A}=2.63 \mathrm{~mA}$

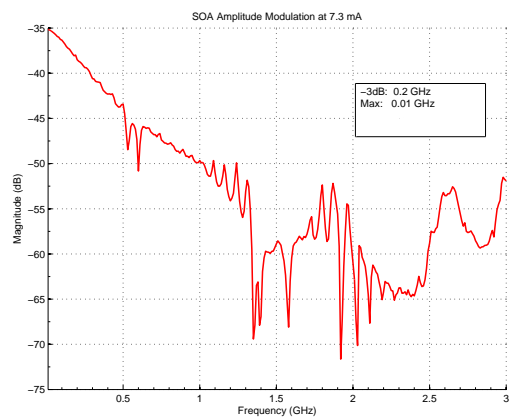

(d) $I_{S O A}=7.30 \mathrm{~mA}$

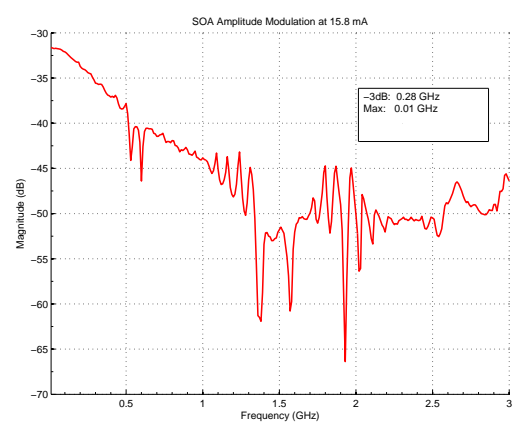

(f) $I_{S O A}=15.80 \mathrm{~mA}$



(h) $I_{S O A}=31.00 \mathrm{~mA}$ 


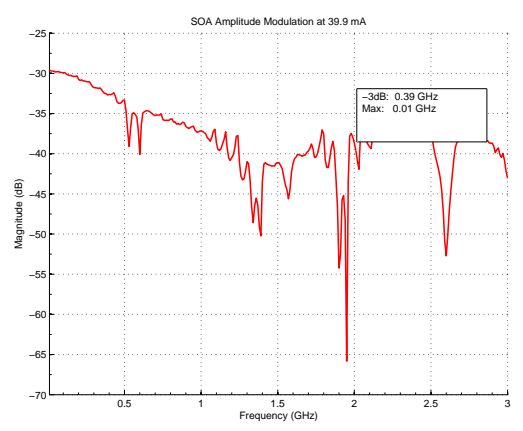

(i) $I_{S O A}=39.90 \mathrm{~mA}$

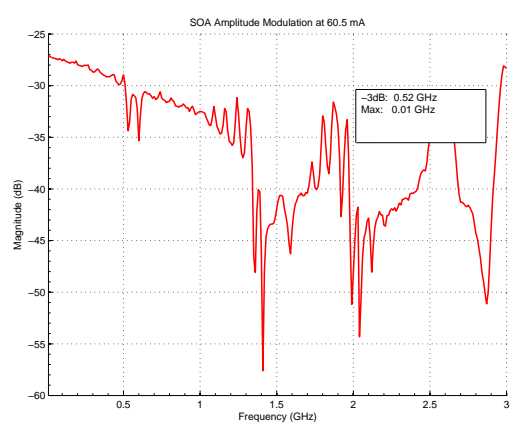

(k) $I_{S O A}=60.50 \mathrm{~mA}$



(m) $I_{S O A}=80.70 \mathrm{~mA}$

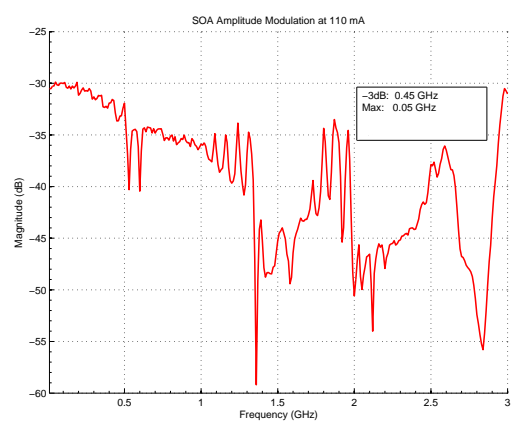

(o) $I_{S O A}=110.00 \mathrm{~mA}$

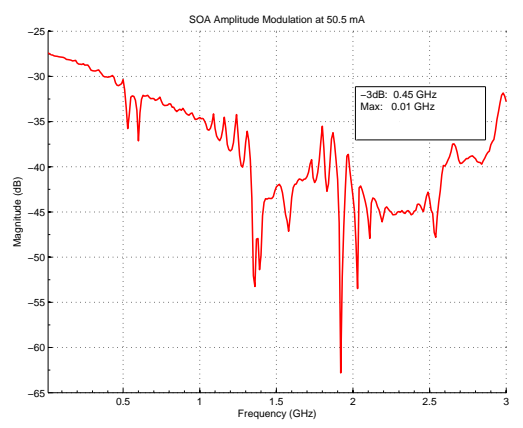

(j) $I_{S O A}=50.50 \mathrm{~mA}$

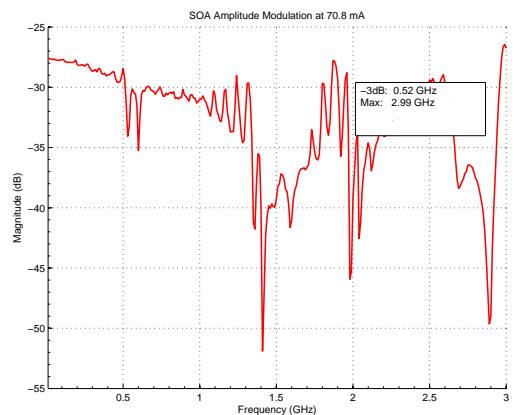

(l) $I_{S O A}=70.80 \mathrm{~mA}$

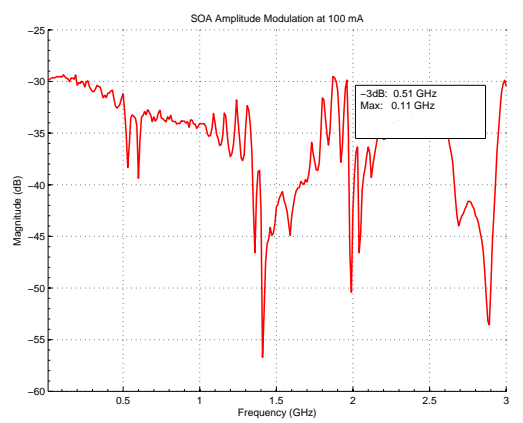

(n) $I_{S O A}=100.00 \mathrm{~mA}$

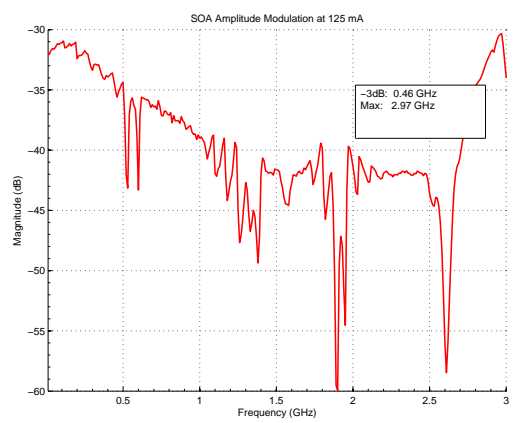

(p) $I_{S O A}=125.00 \mathrm{~mA}$ 


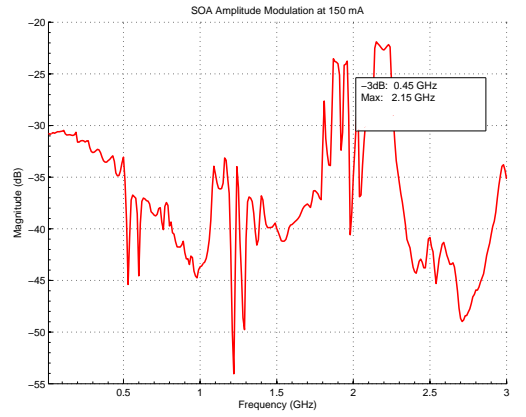

(q) $I_{S O A}=150.00 \mathrm{~mA}$

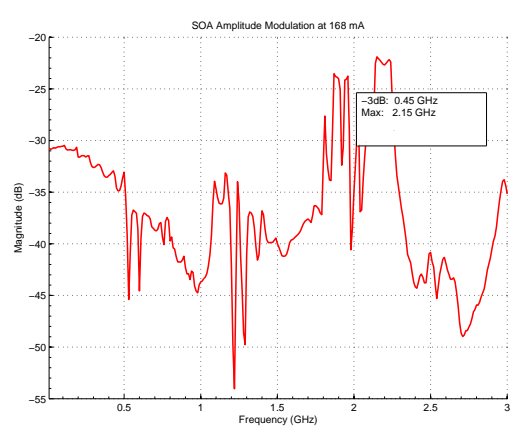

(r) $I_{S O A}=168.00 \mathrm{~mA}$

Figure E.2: AM Response $\left(S_{21}\right)$ of Varying SOA Bias. Current is varied from $I_{S O A}=0.00-168.00 \mathrm{~mA}$ in $(\mathrm{a})-(\mathrm{r})$; Bias conditions are $I_{P M}=$ $I_{B M}=I_{F M}=0 \mathrm{~mA}$, and $I_{G A I N}=100 \mathrm{~mA}$. The $-3 \mathrm{~dB}$ frequency is presented in the box of each subplot. The optimal bias is $60-100 \mathrm{~mA}$ for $>500 \mathrm{MHz}$ bandwidth. 